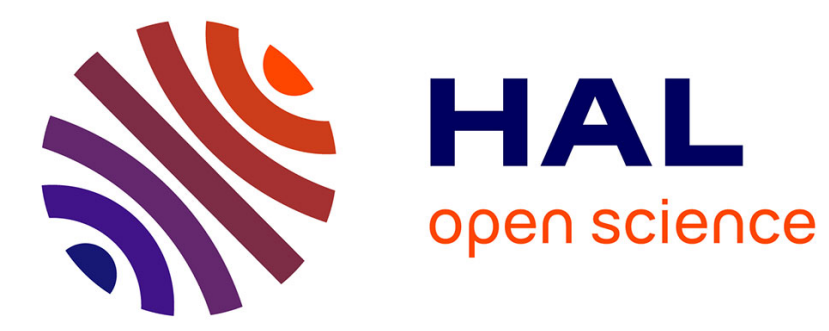

\title{
Refractive Index of Optical Materials
}

Jean-Louis Meyzonnette, Jacques Mangin, Michel Cathelinaud

\section{To cite this version:}

Jean-Louis Meyzonnette, Jacques Mangin, Michel Cathelinaud. Refractive Index of Optical Materials. Springer Handbook of Glass, pp.997-1045, 2019, Springer Handbooks book series (SHB), 10.1007/9783-319-93728-1_29. hal-02405126

\section{HAL Id: hal-02405126 \\ https://hal-univ-rennes1.archives-ouvertes.fr/hal-02405126}

Submitted on 11 Dec 2019

HAL is a multi-disciplinary open access archive for the deposit and dissemination of scientific research documents, whether they are published or not. The documents may come from teaching and research institutions in France or abroad, or from public or private research centers.
L'archive ouverte pluridisciplinaire HAL, est destinée au dépôt et à la diffusion de documents scientifiques de niveau recherche, publiés ou non, émanant des établissements d'enseignement et de recherche français ou étrangers, des laboratoires publics ou privés. 


\section{CHAPTER 1}

\section{Refractive index of optical materials}

${ }^{1}$ Jean-Louis Meyzonnette, ${ }^{2}$ Jacques Mangin and ${ }^{3}$ Michel Cathelinaud

${ }^{1}$ on leave from: Institut d'Optique graduate school, 91127, Palaiseau, France

${ }^{2}$ on leave from: Laboratoire Interdisciplinaire Carnot de Bourgogne, 21078, Dijon, France

${ }^{3}$ Sciences Chimiques de Rennes, UMR CNRS 6226, Université Rennes 1, 35042 Rennes, France 
This chapter deals with the use of methods for measuring the refractive index of optical materials. It contains five sections:

The first section recalls some bases of the electromagnetic theory of light leading to the main characteristics of the index of refraction, and their consequences in geometrical optics (Snell-Descartes laws), in the spectral transmission and absorption of optical media, or the polarization of light beams at interfaces between optical media.

The second section describes the more or less classical panel of methods that have been devised to measure refractive indices of bulk materials: these are essentially based upon either the refraction or reflection of light inside prisms (minimum deviation angle, Littrow methods,...) polarizing properties of optical boundaries (ellipsometric, Brewster configurations).

While the previous section consists of refractive index characterization at a given temperature, the third section is dedicated to the dependence of the refractive index upon the temperature: the normalized thermo-optic coefficient (NTOC) is defined here and an experimental set-up specially designed for this purpose by one of the authors is described in detail.

The last section is concerned with the fact that most optical components are thin film coated in order to improve their performances, in transmission, reflection or absorption. Since spectrophotometry is extensively used to characterize these coatings, the operating principle of spectrophotometers is recalled, as well as the main parameters of these deposited films that one can expect to extract by using this technology from spectrophotometric measurements. Also various spectrophotometric procedures are described to determine the optical constants of optical "systems" (bulk and thin film compounds) in the case of homogeneous or inhomogeneous films, slightly absorbing or not. 


\section{Contents}

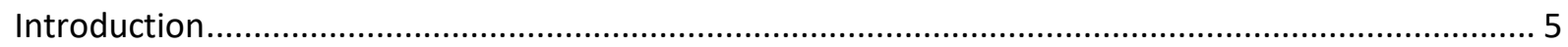

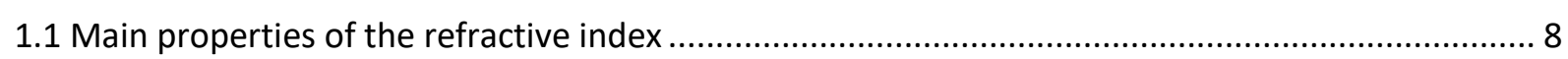

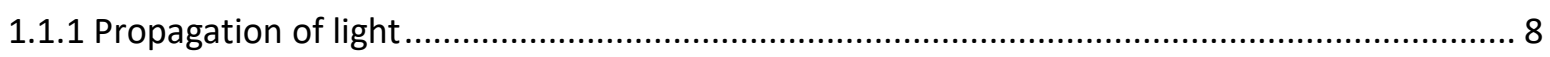

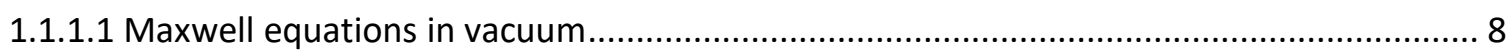

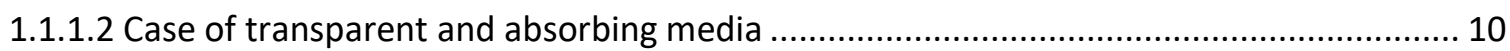

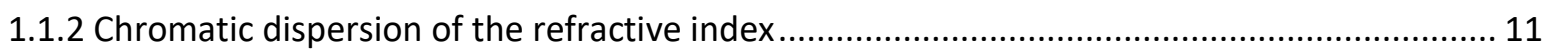

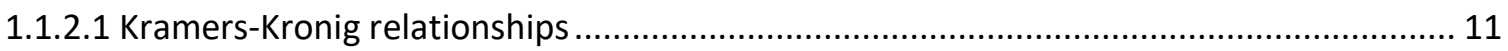

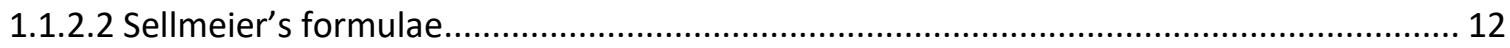

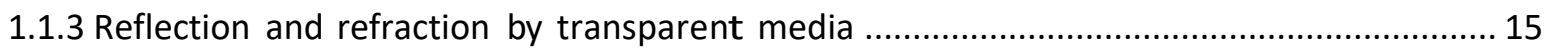

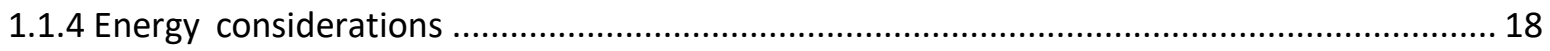

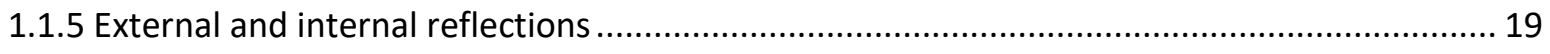

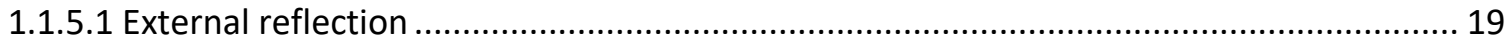

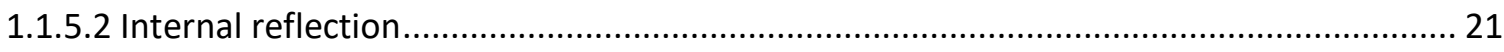

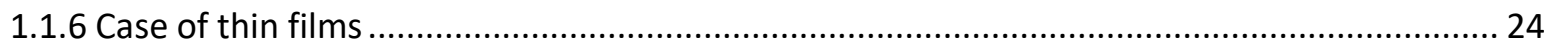

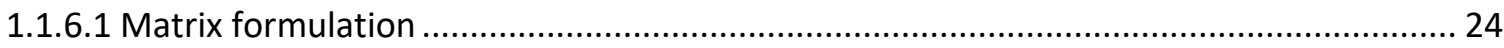

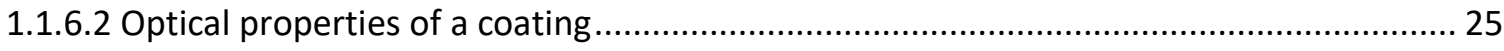

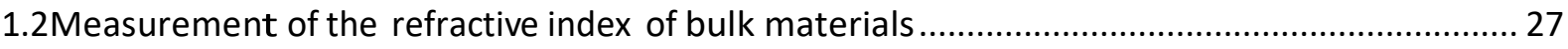

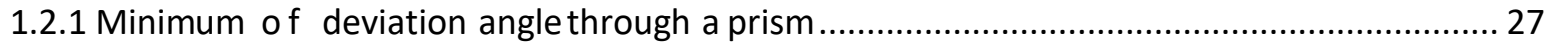

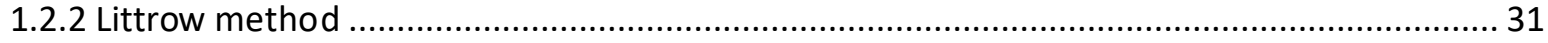

1.2.3 Methods based upon grazing incidence and total internal reflection .............................. 31

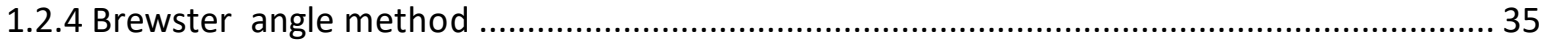

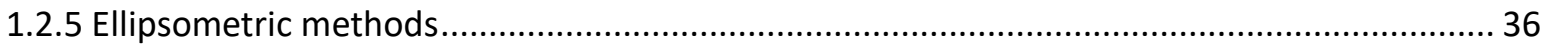

1.2.5.1 Operating principle and measurement parameters ........................................................ 36

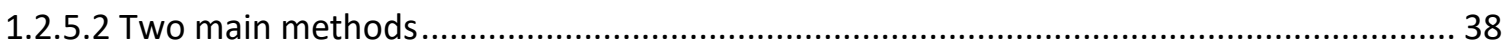

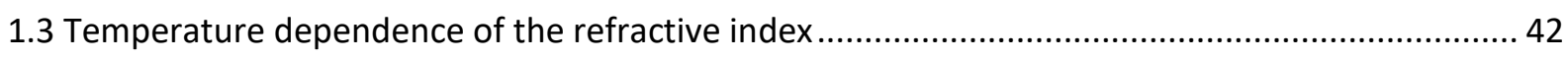

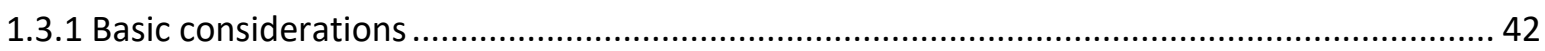

1.3.2 Measurement of the temperature dependence of the refractive index $n(\lambda, T) \ldots \ldots \ldots \ldots \ldots \ldots . . . . . . . . .45$

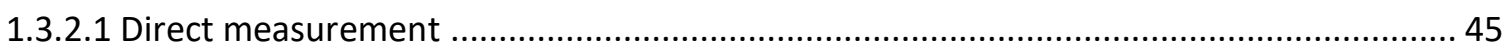

1.3.2.2 Interferometric methods: normalized thermo-optic coefficients (NTOC) ....................... 47

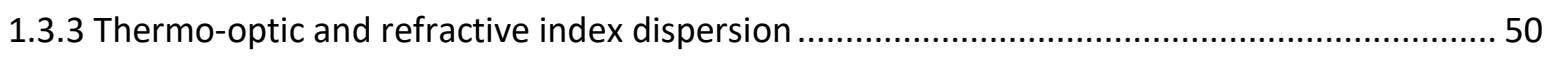

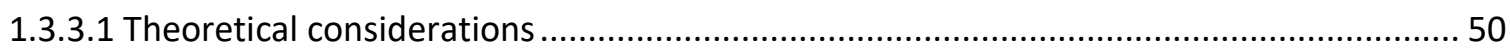

1.3.3.2 Accuracy: temperature dependence of an ONL interaction in $\mathrm{RbTiOPO}_{4} \ldots \ldots \ldots \ldots \ldots \ldots \ldots . . . . . . . . . . . . .53$

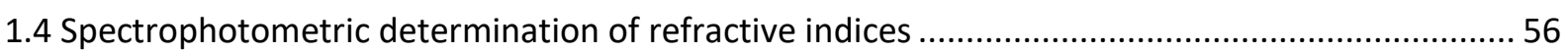

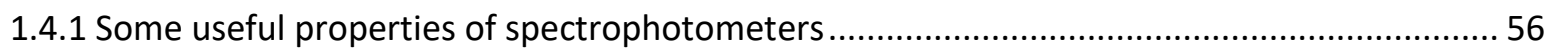


1.4.1.1 Structure and main components of a spectrophotometer

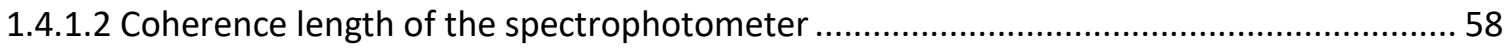

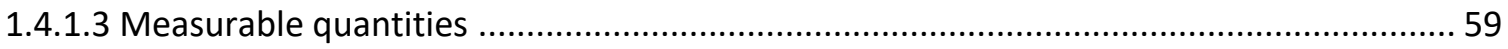

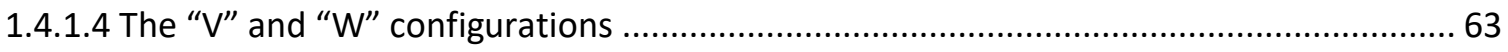

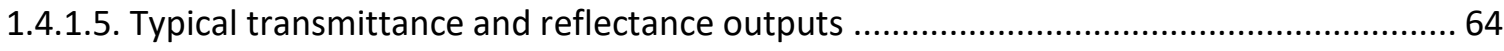

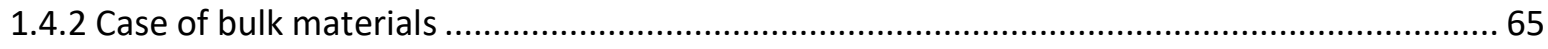

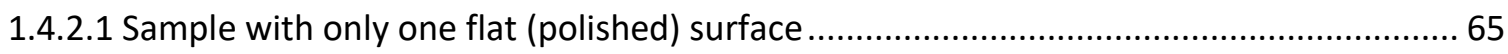

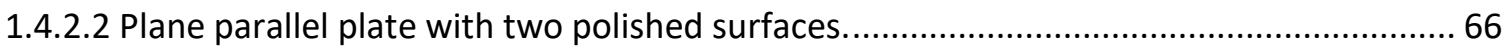

1.4.3 Refractive index measurement of homogeneous dielectric thin films .................................. 67

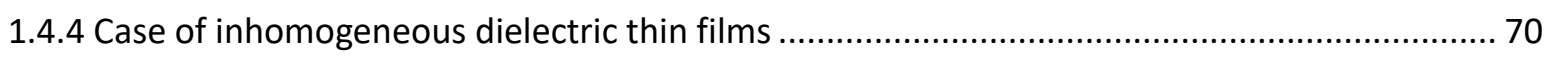

1.4.5 Case of metallic films deposited on a transparent substrate................................................... 72

1.4.6 Optical constant determination by the bilayer « metallic-dielectric » method........................ 73

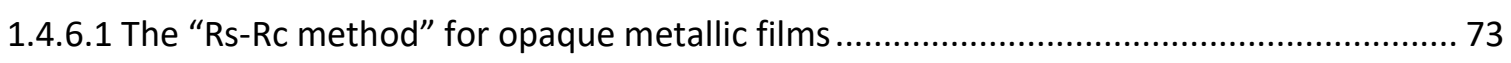

1.4.6.2 Case of metal-dielectric bilayer with non-opaque metallic layers .................................... 76

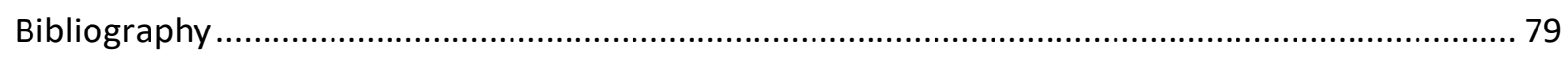

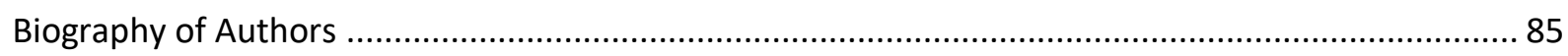




\section{Introduction}

The speed of light in vacuum, designated as c, is a physical constant for any type of electromagnetic radiation. For a monochromatic radiation of frequency $v$, the wavelength $\lambda_{0}$ is then given by the following relationship:

$$
\lambda_{0}=\frac{c}{\mathrm{v}}(1.1)
$$

When light travels through a transparent, homogeneous material, its frequency remains unchanged, but its speed of propagation is modified (reduced) from $c$ to $v$, so that its wavelength becomes $\lambda=\mathrm{v} / v$. The ratio between these quantities is the absolute index of refraction $n$ of the medium: $n=c / \mathrm{v}=\lambda_{0} / \lambda$. If two media, 1 and 2 have respective indices $n_{1}$ and $n_{2}$, the following ratio, $n_{1,2}=n_{1} / n_{2}=\mathrm{v}_{2} / \mathrm{v}_{1}=\lambda_{2} / \lambda_{1}$ is designated as the relative refractive index of the first medium 1 with respect to the second one.

Along with its wavelength, the other parameters of a light beam that are modified by the refractive index of the optical materials it encounters are its direction of propagation, split into both reflected and refracted beams at the interface, the energy it carries (attenuation or absorption by the atoms of the medium), and its state of polarization (direction of vibration). These effects are frequency dependent because they result from the mutual interaction between the incident electromagnetic field and the electrons of the medium under consideration. Frequency dependence, and its effect may be analyzed by means of the basic electromagnetic theory of light.

Among the parameters influencing the refractive index of a medium, one will mention the number of atoms per unit volume, related to its density $\rho_{m}$, and its chemical structure, converted into specific refractivity $R$ in the following $\mathrm{H}$. A. Lorentz formula:

$$
\left(n^{2}-1\right) /\left(n^{2}+2\right)=R \cdot \rho_{m}
$$




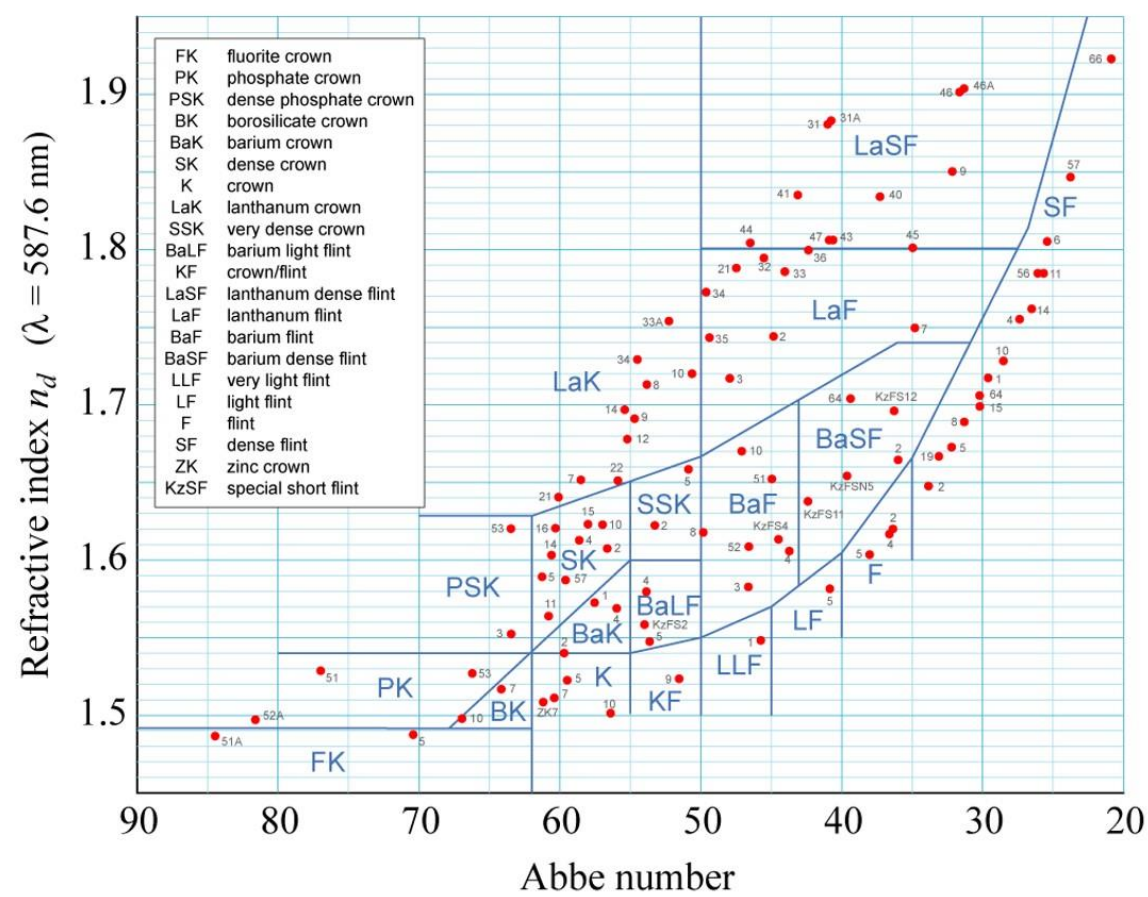

Figure 1.1: Abbe diagram of optical glasses (Schott catalog)[1]

Hence gases have lowest values of refractive index (close to one), as compared to liquids (typically between 1.3 and 1.6) and solids (between 1.3 and 4).

In most experimental set-ups, refractive indices of solids and liquids are not measured in vacuum but in the presence of ambient atmosphere. This consideration must be taken into account for specific applications (aeronautical, space or satellite optical systems), since the result from such measurements is the relative index of the medium with respect to that of air:

$$
n_{\text {relative }}=n_{\text {absolute }} / n_{\text {air }}
$$

In the visible, the refractive index of air is around 1.00029, so the relative difference between absolute and relative indices is about $3.10^{-4}$

The specific refractivity $R$ of a medium depends upon the frequency of the incoming light. This defines the dispersion of the medium, i.e. the change of the refractive index with respect to wavelength $\lambda$ or (less generally) frequency $v$ or wave-number $1 \&=1 / \lambda$.

In the visible domain (380 $\mathrm{nm}<\lambda<780 \mathrm{~nm}$ ), glasses are generally specified by the value of their refractive index $n_{d}$ near the center of the domain ( $\lambda=587.6 \mathrm{~nm}$ at the $d$ spectral line of $\mathrm{He}$ ) and their dispersion by the Abbe number, defined as :

$$
\text { Abbe number }=\left(n_{\mathrm{d}}-1\right) /\left(n_{\mathrm{F}}-n_{\mathrm{C}}\right)
$$


All optical glasses are classified in the Abbe diagram (figure 1.1), where $\left(n_{F}-n_{C}\right)$ is the difference of indices between two Hydrogen spectral lines: $F$ (in the blue, $\lambda=486 \mathrm{~nm}$ ) and $C$ (in the red: $\lambda=656 \mathrm{~nm}$ ).

The influence of temperature upon the refractive index of a medium is characterized by its thermo-optic coefficient, $d n / d T$, i.e. the change of refractive index per degree Celsius. This particular point will be treated in Section 1.2. Pressure also plays a role on the refractive index of media, particularly in gases, for which the molecular density is proportional to the pressure. This also affects liquids to a lesser degree. As far as solids are concerned, large pressure stresses and strains may induce birefringence in the medium, i.e. different values of refractive index depending upon the state of polarization of the incoming light.

Accuracy requirements: Because of its numerous influences upon the behavior of any medium with respect to incident light, an accurate evaluation of its refractive index is of prime importance in all areas of optics: optical design, imaging and non-imaging applications, optical telecommunications, laser optics, atmosphericand space optics, bio-photonics, thin film coatings. To give a few examples:

\section{- Optical telecommunications}

Optical telecommunications are based upon beam guiding by means of total reflection inside step index fibers or gradient index fibers that necessitate perfect refractive index monitoring.

\section{- Low attenuation coatings}

As optical systems become more and more complex and involve an increasing number of components, reflection phenomena at each interface must be reduced to minimal values. This is generally achieved by depositing thin films coatings with refractive indices known as accurately as possible.

\section{- Optical design}

In the simple case of a monochromatic system (at some wavelength $\lambda$ ) made up of a thin lens, its focal length $f$ is given by the well-known formula:

$$
\frac{1}{f}=(n-1)\left(\frac{1}{r_{1}}-\frac{1}{r_{2}}\right)(1.5)
$$

where $r_{1}$ and $r_{2}$ are the radii of curvature of the front and back surfaces, respectively, and $n$ the refractive index at that wavelength $\lambda$. Hence, for a monochromatic system, the uncertainty $\sigma_{n}$ upon the value of therefractive index induces the following uncertainty $\sigma_{f}$ upon the focal length $f$ :

$$
\frac{\sigma_{f}}{f}=\frac{\sigma_{n}}{(n-1)}(1.6)
$$

In more complex optical systems, close to the diffraction limit, the uncertainties on the refractive indices of the various optical components over some spectral domain (visible, near IR, or thermal IR) must be low enough so as to maintain the root mean square ( $\mathrm{rms}$ ) RMS) value of the optical path difference (OPD) induced by chromatic aberration onto the output wave-front. Quality criteria like 
Maréchal's or Strehl's ones are generally required (typically: OPDrms $<\lambda / 1 O$ ). Without getting into too much detail, one may show that this condition leads to the following constraint upon the needed accuracy of refractive index measurement, at each wavelength:

$$
\sigma_{n}=K(n-1) \lambda_{\text {average }}
$$

\begin{tabular}{|c|c|c|c|c|}
\hline Refractive index & $\sigma_{n}$ Visible & $\sigma_{n}$ Near IR & $\sigma_{n} 3 \mu \mathrm{m}-5 \mu \mathrm{m}$ & $n \sigma_{n} 8 \mu \mathrm{m}-12 \mu \mathrm{m}$ \\
\hline & $\lambda_{\text {average }}=0.5 \mu \mathrm{m}$ & $\lambda_{\text {average }}=1.5 \mu \mathrm{m}$ & $\mathrm{n} \lambda_{\text {average }}=4 \mu \mathrm{m}$ & $\lambda_{\text {average }}=10 \mu \mathrm{m}$ \\
\hline$n=1.5$ & $1.10^{-6}$ & $3.10^{-6}$ & & \\
\hline$n=2$ & & & $2.10^{-5}$ & $5.10^{-5}$ \\
\hline$n=4(\mathrm{Ge})$ & & & $6.10^{-5}$ & $1.5 .10^{-4}$ \\
\hline
\end{tabular}

Table 1.1: Specifications on refractive index accuracy of bulk materials for high quality optical systems

In equation (1.7), $K$ is a coefficient taking into account the system complexity (number and thicknesses of refractive components), $\lambda_{\text {average }}$ and $n$ are the average values of wavelength and refractive index over the spectral domain. For high resolution systems representative of aeronautical and space applications, a value of $K$ close to $5.10^{-6} \mu \mathrm{m}^{-1}$ is appropriate if the average wavelength is expressed in $\mu \mathrm{m}$ units. Table 1.1 lists some orders of magnitude of the refractive index accuracy that is required by optical designers for high quality systems, with respect to average values of wavelengths and refractive indices.

\section{- Atmospheric corrections:}

For applications in which long distances of atmosphere are being traversed by light, tiny fluctuations of refractive index induced by local temperature changes produce phenomena such as turbulence.

- Optical range-finding:

High precision distance measurements, which are based upon time- of-flight range-finding techniques, are degraded by uncertainties on refractive index of the intermediate medium. This is the case for instance in astronomical experiments about the precise measurement of the distance between the Earth and the Moon.

\subsection{Main properties of the refractive index}

\subsubsection{Propagation of light}

\subsubsection{Maxwell equations in vacuum}

These equations set relationships between the electric and magnetic fields of a radiation propagating inside a medium. Written as follows in the SI international set of units, they are the starting point for evaluating the refractive index of the material: 


$$
\begin{gathered}
\nabla . \boldsymbol{D}=\rho \\
\nabla \times \boldsymbol{E}=-\frac{\partial \boldsymbol{B}}{\partial t} \\
\nabla . \boldsymbol{B}=0 \quad \text { (1.8a) } \\
\nabla \times \boldsymbol{H}=\boldsymbol{j}+\frac{\partial \boldsymbol{D}}{\partial t} \\
\text { with } \nabla . \boldsymbol{D}=\frac{\partial D}{\partial x}+\frac{\partial D}{\partial y}+\frac{\partial D}{\partial z}(1.8 \mathrm{~b}) \\
\text { and the vector } \nabla \times \boldsymbol{E}=\left(\frac{\partial E_{z}}{\partial y}-\frac{\partial E_{y}}{\partial z}, \frac{\partial E_{x}}{\partial z}-\frac{\partial E_{z}}{\partial x}, \frac{\partial E_{y}}{\partial x}-\frac{\partial E_{x}}{\partial y}\right) \quad(1.8 \mathrm{c})
\end{gathered}
$$

where $\boldsymbol{E}$ is the electric field vector, $\boldsymbol{D}$ the electric displacement field, $\boldsymbol{H}$ the magnetic field, $\boldsymbol{B}$ the magnetic induction, $\rho$ the free electric charge density and $j$ the free current density. In the case of dielectric, homogeneous media such as a good quality glass, $j=\rho=0$, and hence Maxwell's equations reduce to:

$$
\begin{aligned}
& \nabla . \boldsymbol{E}=0 \\
& \nabla \times \boldsymbol{E}=-\mu \frac{\partial \boldsymbol{H}}{\partial t} \\
& \nabla . \boldsymbol{H}=0 \\
& \nabla \times \boldsymbol{H}=\varepsilon \frac{\partial \boldsymbol{E}}{\partial t}
\end{aligned}
$$

where $\varepsilon$ and $\mu$ are the dielectric permittivity and magnetic permeability, respectively. By taking the derivative of the last equation with respect to time, one obtains:

$$
\nabla \times \frac{\partial \boldsymbol{H}}{\partial t}=\varepsilon \frac{\partial^{2} \boldsymbol{E}}{\partial t^{2}}
$$

Then, by taking the curl of second equation, there comes:

$$
\nabla \times(\nabla \times \boldsymbol{E})=-\mu \nabla \times\left(\frac{\partial \boldsymbol{H}}{\partial t}\right)=-\varepsilon \mu \frac{\partial^{2} \boldsymbol{E}}{\partial t^{2}}
$$

Noting that $\Delta \cdot A=\nabla \cdot(\nabla \cdot A)-\nabla \times(\nabla \times A)=\nabla^{2} A$ and taking into account the fact that $\nabla \cdot \boldsymbol{E}=0$ we find:

$$
\Delta \cdot \boldsymbol{E}=\frac{1}{\mathrm{v}^{2}} \frac{\partial^{2} \boldsymbol{E}}{\partial t^{2}} \text { with } \mathrm{v}=\frac{1}{\sqrt{\varepsilon \mu}}
$$


This is the equation of propagation of the electric field $\boldsymbol{E}$ (the same result can be derived concerning the magnetic field) leading to the value of the speed of propagation of light and which is, in vacuum:

$$
c=\frac{1}{\sqrt{\varepsilon_{0} \mu_{0}}}=3.10^{8} \mathrm{~m} / \mathrm{s}, \text { since } \varepsilon_{0}=1 / 36 \pi \mathrm{nF} / \mathrm{m} \text { and } \mu_{0}=4 \pi \cdot 10^{-7} \mathrm{H} / \mathrm{m} \text {. }
$$

In a transparent medium other than vacuum, one gets:

$$
\varepsilon=\varepsilon_{r} \varepsilon_{0} \quad \text { and } \mu=\mu_{r} \mu_{0}
$$

For dielectric materials such as glasses, $\mu_{r} \sim 1$, and hence there comes:

$$
n=\frac{c}{\mathrm{v}}=\sqrt{\varepsilon_{r}}(1.14)
$$

which corresponds in optics to the so-called refractive index $n$ of the medium.

\subsubsection{Case of transparent and absorbing media}

It is convenient to take the refractive index as a complex parameter, and going back to Maxwell's equations (1.8), complex solutions in terms of plane waves (in z-direction), sinusoidally modulated with respect to time $t$ with angular frequency $\omega$ are written in the form:

$$
\bar{E}(z)=E_{0} e^{-\frac{\alpha z}{2}} e^{i\left[\omega\left(t-\frac{\bar{n} z}{c}\right)+\varphi\right]}(1.15)
$$

where the complex refractive index is set as $\bar{n}=n-i \kappa(1.16)$; similarly, the complex absolute dielectric permittivity of the medium will be written as $\bar{\varepsilon}=\bar{n}^{2}=\varepsilon^{\prime}-i \varepsilon^{\prime \prime}(1.17)$. The solution (1.15) is referred to the simple case of a harmonic wave propagating along the $z$ axis in an isotropic medium, $\boldsymbol{E}$ being perpendicular to $\boldsymbol{H}$ and both lying in a plane normal to the $z$ direction. $\varphi$ stands for a phase at origin $t=0$ and $z=0$. The coefficient $\alpha$ characterizes the absorption of the electromagnetic wave at angular frequency $\omega$ and is determined from spectroscopic measurements.

Now, following Beer-Lambert's law, the intensity $I(z)$ of a beam after a propagation length $z$ in an optical material is given by:

$$
I(z)=I_{0} e^{-\alpha z}
$$

where $I_{0}$ is the intensity of the incident light. Notice also that in terms of electric field attenuation, formula (1.18) corresponds to a field $E(z)$ :

$$
E(z)=E_{0} e^{-\frac{\alpha z}{2}}
$$

since $I(z) \propto|E(z)|^{2}, E_{0}$ being a real.

Hence, the basic equations that can be drawn for non-conductive and non-magnetic media are: 


$$
\begin{array}{r}
\alpha=\frac{2 \kappa \omega}{c}=\frac{4 \pi \kappa}{\lambda}(1.20) \\
n^{2}-\kappa^{2}=\varepsilon^{\prime} ; \quad 2 n \kappa=\varepsilon^{\prime \prime}
\end{array}
$$

$n$ is the real refractive index which obeys to the Snell-Descartes's relationships, while $\kappa$-is the so-called extinction coefficient; both depend on the angular frequency $\omega$. The complex dielectric function or relative dielectric permittivity $\overline{\varepsilon_{r}}$ is written as $\overline{\varepsilon_{r}}=\bar{\varepsilon} / \varepsilon_{0}=\left(\varepsilon^{\prime}-i \varepsilon^{\prime \prime}\right) / \varepsilon_{0}$ (1.22).

\subsubsection{Chromatic dispersion of the refractive index}

\subsubsection{Kramers-Kronig relationships}

One of the main ways useful to determine the real and imaginary parts of the complex refractive index is to perform spectroscopic measurements over the whole frequency range, that is from $\omega=0$ to $\omega \rightarrow \infty$.

Kramers-Kronig (K-K) relations derive from the causality principle generally admitted in physics, which states that the response of a system submitted to a given excitation cannot exist before this excitation has taken place [2]. The system is supposed to be homogeneous, stable in time, and exhibiting a linear response. Basically, if $f(t)$ represents the response in time domain, the causality principle states that we must have:

$$
f(t)=0 \quad \forall t<0
$$

One method for obtaining the K-K relations is to start by multiplying the $f(t)$ function by the Heaviside $H(t)$ step one, which does not change anything to relation (1.23), and going then to the frequency domain by taking the Fourier transform $\mathrm{F}(\omega)$ of the product:

$$
\mathrm{F}(\omega)=\frac{1}{\sqrt{2 \pi}} \int_{-\infty}^{+\infty} f(t) \cdot H(t) e^{-i \omega t} d t
$$

Detailed calculations following formula $(1.24)$ can be found in ref $[3,4]$ for instance. It comes out that in terms of frequency dependence of the real and imaginary parts of the dielectric constant, the $\mathrm{K}-\mathrm{K}$ relations may be expressed as:

$$
\begin{aligned}
& \varepsilon^{\prime}(\omega)-1=\frac{2}{\pi} P \int_{0}^{\infty} \frac{\omega^{\prime} \varepsilon^{\prime \prime}\left(\omega^{\prime}\right)}{\omega^{\prime 2}-\omega^{2}} d \omega^{\prime} \\
& \varepsilon^{\prime \prime}(\omega)=-\frac{2 \omega}{\pi} P \int_{0}^{\infty} \frac{\varepsilon^{\prime}\left(\omega^{\prime}\right)-1}{\omega^{\prime 2}-\omega^{2}} d \omega^{\prime}
\end{aligned}
$$

where $P$ denotes the Cauchy principal value of the improper integrals of rational functions $\mathrm{g}\left(\omega^{\prime}\right)$ : 


$$
P \int_{0}^{\infty} \mathrm{g}\left(\omega^{\prime}\right) d \omega^{\prime} \equiv \lim _{\xi \rightarrow 0}\left[\int_{0}^{\omega-\xi} \mathrm{g}\left(\omega^{\prime}\right) d \omega^{\prime}+\int_{\omega+\xi}^{\infty} \mathrm{g}\left(\omega^{\prime}\right) d \omega^{\prime}\right]
$$

In practice, for bulk optical materials, the refractive index $n$ and extinction coefficient $\pi$ are deduced from the reflection spectra performed under (nearly) normal incidence. From Fresnel formula $R=r(\omega) r^{*}(\omega)$ represents by definition the normal reflectance. The complex reflection coefficient $r(\omega) \underline{\text { undergoes a phase change }} \underline{\varphi(\omega) \text { and is written as }} r(\omega)=|r(\omega)| e^{-i \varphi(\omega)}$ where $|r(\omega)| \underline{\text { is the }}$ complex reflectivity modulus. As a result of K-K relations we have:

$$
\varphi(\omega)=\frac{2 \omega}{\pi} P \int_{0}^{\infty} \frac{\omega \ln r\left(\omega^{\prime}\right)}{\omega^{2}-\omega^{\prime 2}} d \omega^{\prime}
$$

The refractive index and extinction coefficient can finally be derived from spectroscopic measurement through the use of Fresnel formulae in normal incidence:

$$
\begin{aligned}
& n(\omega)=\frac{1-r^{2}(\omega)}{1+r^{2}(\omega)-2 r(\omega) \cos \varphi(\omega)} \\
& \kappa(\omega)=\frac{2 r(\omega) \sin \varphi(\omega)}{1+r^{2}(\omega)-2 r(\omega) \cos \varphi(\omega)}
\end{aligned}
$$

Thus, reflectance spectra allow calculating $r(\omega)$ and the phase change $\varphi(\omega)$ undergone by the reflected beam. This holds for the case of an optically polished flat front face of a bulk sample which is exposed to an impinging beam and set in a free space (nearly vacuum condition).

From a technical point of view, care must be taken to avoid an eventual contribution of the rear face of the sample to the reflected intensity; this can be realized for instance by shaping the material in a slightly wedged form. The case of a double sided polished sample is examined in section 1.4.2.2

\subsubsection{Sellmeier's formula}

This subsection is intended only to recall the basic equations that have led to the dispersion formula of refractive index, in view of using them further in Section 1.3.3 for a rigorous formulation of their temperature dependence.

Maxwell equations (see \$1.1.1) are the constitutive formula that describe at a macroscopic scale the response of a dielectric medium to an applied electric field $\boldsymbol{E}$, whatever be the atomic structure of the material. Dealing with refractive index determination, the main parameter that is to be taken into account is the absolute complex permittivity $\bar{\varepsilon}$ of the medium which is related to the polarization $\boldsymbol{P}$ induced by the field: $\boldsymbol{P}=\varepsilon_{0}\left(\overline{\varepsilon_{r}}-1\right) \boldsymbol{E}$ (1.31).

From a microscopic analysis, the Lorentz's model introduces the mean molecular polarizability $\alpha_{p} \sigma$ and assumes to first order that the polarization $\boldsymbol{P}$ at a mesoscopic scale is the summation of the 
mean polarizability of each molecule forming the material over the unit volume of matter. For weak fields, to a first approximation, the polarization $\boldsymbol{P}$ induced by a field $\boldsymbol{E}$ is supposed to be linear, described by the simplest form $\boldsymbol{P}=\varepsilon_{0} \bar{\chi} \boldsymbol{E}$ (1.32) where $\bar{\chi}$ is defined here as the complex dielectric susceptibility of the material, related to the above mentioned permittivity by the straightforward relationship $\bar{\chi}=1-\overline{\varepsilon_{r}}(1.33)$.

Speaking now in terms of mean molecular polarizability $\alpha_{p}$, it was shown on another hand that the effective field $\boldsymbol{E}$ ' acting on a molecule (Lorentz's local field) is given by $\boldsymbol{E}^{\prime}=\boldsymbol{E}+\frac{\boldsymbol{P}}{3 \varepsilon_{0}}$ (1.34), larger than the average electric field $\boldsymbol{E}$ in the dielectric [5]. From these considerations, the mean molecular polarizability $\alpha_{P} \sigma$ was related to the dielectric constant $\varepsilon$ (or the square of the refractive index $n$ in Maxwell theory) through the so-called Lorentz-Lorenz formula:

$$
\alpha_{p}=\frac{3 \varepsilon_{0}}{N} \frac{\varepsilon-1}{\varepsilon+2}=\frac{3 \varepsilon_{0}}{N} \frac{n^{2}-1}{n^{2}+2}
$$

where $N$ is the number of molecules per unit volume. The corresponding total electric moment $\boldsymbol{P}$ is $\boldsymbol{P}=N \boldsymbol{p}=N \alpha_{P} \boldsymbol{E}^{\prime}$ (1.36) where $\boldsymbol{p}$ is the molecular elementary electric moment.

For interaction of light with matter, we have to account for the frequency dependence of the response to an applied harmonic field $\boldsymbol{E}$ of angular frequency $\omega$. A molecule which consists of heavy nuclei surrounded by light particles (electrons) will undergo a local electric field $\boldsymbol{E}$ ', as mentioned above. Assuming no motion of the nuclei, $\boldsymbol{E}$ ' will generate a harmonic motion of the electrons, bonded to the nuclei through a restoring force of amplitude $F_{1}$ proportional to the displacements $x$ from their equilibrium position (Hooke's law):

$$
F_{1}=-k_{f} x(1.37)
$$

where $k_{f} \hbar$ is the restoring constant.

Considering for example one electron, this one will be submitted also to the Lorentz's force $F_{2}$ proportional to its electric charge $e$ and the local field $E^{\prime}$ :

$$
F_{2}=e E^{\prime}
$$

If $m_{e}$ is $m$ the mass of the electron and neglecting the existence of a damping term (resisting force), due to energy dissipation and some kind of friction between atoms, the equation of motion will take the simplest form:

$$
m_{e} \frac{\partial^{2} x}{\partial t^{2}}+k_{f} x=e E^{\prime}
$$


For a harmonic field of the form $E^{\prime}=E_{0}{ }^{\prime} e^{-i \omega t}(1.40)$ and looking to a solution $x=x_{0} e^{-i \omega t}$ (1.41), we get:

$$
x=\frac{e E^{\prime}}{m_{e}\left(\omega_{0}^{2}-\omega^{2}\right)}
$$

where $\omega_{0}=\sqrt{\frac{k_{f}}{m_{e}}}(1.43)$ is the resonance angular frequency of the un-driven electron.

The elementary moment $p=e . x$ is to be extended up to the scale of the unit volume, and summing these contributions gives the total polarization $P=N e . x=N \frac{e^{2}}{m_{e}} \frac{E^{\prime}}{\left(\omega_{0}^{2}-\omega^{2}\right)}$ (1.44).

Combining equations (1.35), (1.42) and (1.44) will give the variation of the refractive index $n$ with angular frequency $\omega$, that is chromatic dispersion, described by:

$$
\frac{n^{2}-1}{n^{2}+2}=\frac{N e^{2}}{3 \varepsilon_{0} m_{e}\left(\omega_{0}^{2}-\omega^{2}\right)}
$$

Of course, at the scale of a unit volume of matter, one may presume the occurrence of many resonance frequencies similar to the simplest case described above, so that formula (1.45) should reasonably be read as:

$$
\frac{n^{2}-1}{n^{2}+2}=\frac{e^{2} N}{3 \varepsilon_{0} m_{e}} \sum_{i} \frac{f_{i}}{\left(\omega_{i}^{2}-\omega^{2}\right)}
$$

where $N f_{i}$ is the number of electrons oscillating at resonance angular frequency $\omega_{i}$.

From formula (1.46) it was shown [5] that the frequency dependence of the refractive index $n$ is described through the so-called Sellmeier's dispersion formula:

$$
\begin{gathered}
n^{2}-1=\sum_{i} \frac{\rho_{i}}{\omega_{i}^{2}-\omega^{2}} \text { (1.47) } \\
\text { where we set } \rho_{i}=\frac{N f_{i} e^{2}}{3 \varepsilon_{0} m_{e}}(1.48)
\end{gathered}
$$

In the above description, only electrons were considered, assuming that they easily move with respect to applied field because of the low value of their mass $m_{e}$; formula (1.47) was proven quite satisfying in the case of gases and particularly at short wavelengths. However, going towards infrared wavelengths we have to take into account the motion of nuclei, and formula (1.47) must also include 
resonance frequencies specific to lattice vibrations of optical materials [6]. Thus two sets of terms appear generally in the Sellmeier's dispersion formula used to predict the value of the refractive index of a compound over its spectral region of transparency. High values of $\omega_{i}$ account for the UV cut-off, while lower ones account for the infrared edge beyond which the compound becomes opaque. In most cases two poles added to a constant term are quite sufficient for describing accurately the chromatic dispersion.

\subsubsection{Reflection and refraction by transparent media}

\section{Snell-Descartes relationships}

Let us consider a monochromatic, plane polarized wave of modulus $E_{0}$ that is incident upon the boundary separating two transparent media, 1 and 2, inside which speeds of propagation are respectively $\mathrm{v}_{1}$ and $\mathrm{v}_{2}$ and corresponding refractive indices $n_{1}=c / \mathrm{v}_{1}$ and $n_{2}=c / \mathrm{v}_{2}$. If $\boldsymbol{E}(x, y, z, t)$ is the electric field of the incident wave, $\boldsymbol{E}_{r}(x, y, z, t)$ and $\boldsymbol{E}_{t}(x, y, z, t)$ being respectively the electric fields of the reflected and transmitted waves, one must write the classical continuity equations at the interface, i.e. equality between the tangential components of $\boldsymbol{E}_{\boldsymbol{1}} \boldsymbol{E}_{r}$ and of $\boldsymbol{E}_{t}$ as well as the same ones for the magnetic fields. These equalities must pertain at all times, which means that the frequencies of these three waves must be the same, hence one may omit the effect of time and consider only the complex amplitudes. The plane of incidence is defined by the propagation vector and the normal to the interface. If we choose the xyz axes such that the $x z$ plane is the plane of incidence (see Figure 1.2), the direction cosines of the incident plane wave are then $\alpha_{1}\left(=\frac{k_{1} \cdot u_{x}}{\left|k_{1}\right|}\right) \beta_{1}\left(=\frac{k_{1} u_{y}}{\left|k_{1}\right|}=0\right)$ and $\gamma_{1}\left(=\frac{k_{1} \cdot u_{z}}{\left|k_{1}\right|}\right)$ where $u_{x_{1}}$ $\boldsymbol{u}_{\mathrm{y}}$ and $\boldsymbol{u}_{\mathrm{z}}$ are the unit vector along the $\mathrm{x}, \mathrm{y}, \mathrm{z}$ axis respectively.

The incident electric vibration may be written as follows, in complex notation:

$$
\boldsymbol{E}=\boldsymbol{E}_{0} \exp \left[i k_{1}\left(\alpha_{1} x+\gamma_{1} z\right)\right] \text { with } k_{1}=n_{1} \frac{\omega}{c}=\frac{2 \pi}{\lambda_{1}}
$$

The reflected and transmitted vibration amplitudes will be written:

$$
\begin{aligned}
& E_{r}=r E_{0} \exp \left[i k_{1}\left(\alpha_{1}^{\prime} x+\beta_{1}^{\prime} y+\gamma_{1}^{\prime} z\right)\right] \\
& E_{t}=t E_{0} \exp \left[i k_{2}\left(\alpha_{2} x+\beta_{2} y+\gamma_{2} z\right)\right]
\end{aligned}
$$

where $\alpha^{\prime}{ }_{1}, \beta^{\prime}{ }_{1,} \gamma_{1}^{\prime}$ are the direction cosines of the reflected wave and $\alpha_{2}, \beta_{2}, \gamma_{2}$ are those of the transmitted wave.

with $k_{2}=n_{2} \frac{\omega}{c}=\frac{2 \pi}{\lambda_{2}}$

Writing continuity conditions at the boundary $(z=0)$ leads to the following results: 


$$
\beta_{1}{ }^{\prime}=\beta_{2}{ }^{\prime}=0 \text { and } k_{1} \alpha_{1}=k_{1} \alpha_{1}{ }^{\prime}=k_{2} \alpha_{2}
$$

The first result, on the $\beta$ 's, expresses the fact that the incident, reflected and refracted rays lie in the same plane, i.e. the plane of incidence.

The second result, on the $\alpha$ 's, leads to the well-known relationship:

$$
\sin \left(\theta_{1}\right)=\sin \left(\theta_{1}^{\prime}\right) \text { and } n_{1} \sin \left(\theta_{1}\right)=n_{2} \sin \left(\theta_{2}\right)
$$

where $\theta_{1}, \theta^{\prime}{ }_{1}$, and $\theta_{2}$ are respectively the angles of incidence, of reflection, and of refraction of the beam.

\section{Fresnel equations at an interface}

At the interface between two homogeneous, isotropic, lossless dielectric media, Maxwell equations must be completed by boundary conditions imposing continuity between tangential components of both electric and magnetic fields, i.e.:

$$
\boldsymbol{E}_{1}^{T}=\boldsymbol{E}_{2}^{T} \text { and } \boldsymbol{H}_{1}^{T}=\boldsymbol{H}_{2}^{T}
$$

The mathematical expressions of reflectance and transmittance, that are derived by writing the continuity conditions at the boundary, depend upon the angle of incidence and the orientation of the electric and magnetic vectors with respect to the plane of incidence: we will examine successively the case of plane polarized light with the electric vector firstly perpendicular to the plane of incidence, and then secondly parallel to it.
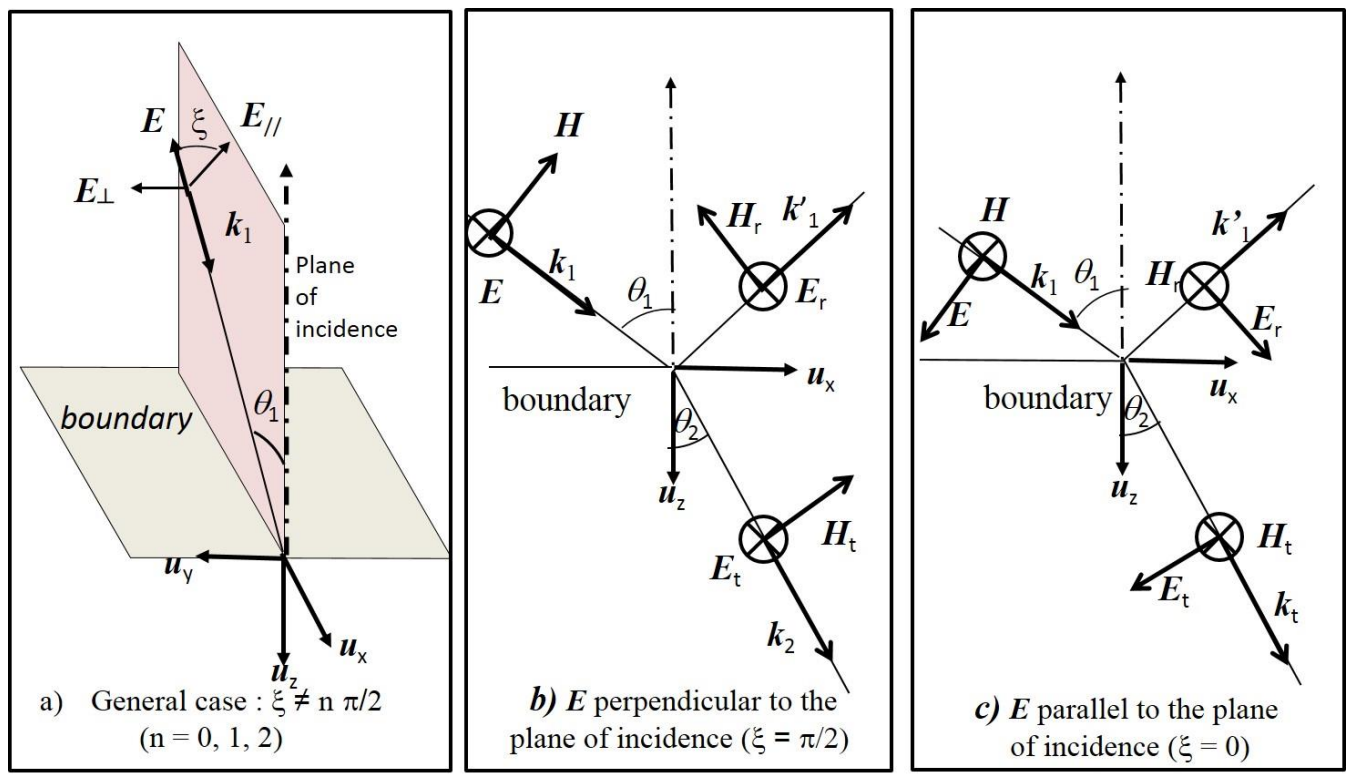

Figure 1.2: Electric, magnetic and propagation vectors: a) general case of linearly polarized Incident wave ; b) transverse electric incident wave ; c) transverse magnetic incident wave. 
$\underline{1^{\text {st }} \text { case: }}$ electric vector perpendicular to the plane of incidence (transverse electric or TE wave):

The possible relative orientations of electric, magnetic and propagation vectors are drawn on figure 1.2. The total tangential component of the electric vector is $\boldsymbol{E}+\boldsymbol{E}_{r}$ in the first medium and $\boldsymbol{E}_{t}$ in the second one (in complex notation). For the magnetic vector, they are $\left(\boldsymbol{H}-\boldsymbol{H}_{r}\right) \cos \left(\boldsymbol{\theta}_{1}\right)$ and $\boldsymbol{H}_{t} \cos \left(\theta_{2}\right)$.

Denoting $r_{\perp}$ the amplitude reflection coefficient (in the literature, one finds also $r_{s}, r_{\mathrm{TE}}$ or $r_{\sigma}$ ) and $t_{\perp}$ (or $\left.\left.t_{s}, t_{\mathrm{TE}}, t_{\sigma}\right)\right)$ the amplitude transmission coefficient, and taking into account the fact that $H=E / \mu \mathrm{v}=$ $n E / \mu c$, noting that the ratio $H / E$ appears as the optical admittance $Y$, one obtains:

$$
1+r_{\perp}=t_{\perp} \quad \text { and }\left(1-r_{\perp}\right) n_{1} \cos \left(\theta_{1}\right)=t_{\perp} n_{2} \cos \left(\theta_{2}\right)
$$

Taking into account the Snell-Descartes's law, that is $n_{1} \sin \left(\theta_{1}\right)=n_{2} \sin \left(\theta_{2}\right)$, this equation system leads to the following results:

$$
r_{\perp}=-\frac{\sin \left(\theta_{1}-\theta_{2}\right)}{\sin \left(\theta_{1}+\theta_{2}\right)} \text { and } t_{\perp}=\frac{2 \cos \left(\theta_{1}\right) \sin \left(\theta_{2}\right)}{\sin \left(\theta_{1}+\theta_{2}\right)}
$$

These two quantities are real, which means that the phase changes at interface may only be 0 or $\pi$. $t_{\perp}$ is always positive (no phase change) and $r_{\perp}$ is negative if $\theta_{1}>\theta_{2}$ (i.e. $n_{2}>n_{1}$ ), or positive if $\theta_{1}<$ $\theta_{2}$ (i.e. $n_{2}<n_{1}$ ). In the case of near normal incidence, these formulas get simpler:

$$
r_{\perp}=\frac{n_{2}-n_{1}}{n_{2}+n_{1}} \text { and } t_{\perp}=\frac{2 n_{1}}{n_{2}+n_{1}}
$$

$\underline{2}^{\text {nd }}$ case: electric vector lying in the plane of incidence (transverse magnetic or TM wave):

If the amplitude reflection and transmission coefficients are denoted $r_{/ /}\left(\right.$or $\left.r_{p}, r_{\pi}\right)$ and $t_{/ /}\left(\right.$or $\left.t_{p}, t_{\pi}\right)$, the orientation of the electric, magnetic and propagation vectors is given on figure 1.2, which leads to the following continuity conditions:

$$
\left(1+r_{/ /}\right) \cos \left(\theta_{1}\right)=t_{/ /} \cos \left(\theta_{2}\right) \text { and }\left(1-r_{/ /}\right) \sin \left(\theta_{2}\right)=t_{/ /} \sin \left(\theta_{1}\right)
$$

and hence to the following expressions for both amplitude reflection and transmission coefficients:

$$
r_{/ /}=-\frac{\tan \left(\theta_{1}-\theta_{2}\right)}{\tan \left(\theta_{1}+\theta_{2}\right)} \text { and } t_{/ /}=\frac{2 \cos \left(\theta_{1}\right) \sin \left(\theta_{2}\right)}{\sin \left(\theta_{1}+\theta_{2}\right) \cos \left(\theta_{1}-\theta_{2}\right)}
$$

Here again, these coefficients are real, hence phase changes can only be 0 or $\pi$. In case of near normal incidence, one finds the same results as for the previous case. 


\subsubsection{Energy considerations}

It must be recalled here that optical metrological instruments are sensitive to optical powers or fluxes (i.e. optical energy flow per unit time), while previous results are derived from reflection and transmission coefficients calculated at the scale of local electric fields $\boldsymbol{E}$. These coefficients have to be converted into corresponding measurable, that is namely energetic quantities. This can be done simply by considering that the energy flow per unit time of a beam of light (i.e. its flux or power $P$ ) is equal to the product of its energy density per unit area, $d W / d S$, times the beam cross section $S$ and the speed of propagation of light, v. Since the energy density per unit area is proportional to $\varepsilon E^{2}, \varepsilon$ being the dielectric constant of the medium involved, the correspondence between powers of the incident beam and either one of the reflected or transmitted beams may be written as:

$$
\frac{P_{2}}{P_{i n c}}=\left(\frac{\frac{d W_{2}}{d S}}{\frac{d W_{i n c}}{d S}}\right) \frac{S_{2}}{S_{1}} \frac{\mathrm{v}_{2}}{\mathrm{v}_{i n c}} \text { (1.59) }
$$

In the case of reflection, incident and reflected beams have identical cross sections and propagate through the same medium (i. e. same dielectric constant $\varepsilon$ and speed of propagation of light), hence: the reflectance $R$ is

$$
R=\frac{P_{r}}{P_{i n c}}=\left(\frac{E_{r}}{E_{\text {inc }}}\right)^{2}=r^{2}(1.60)
$$

For the transmitted beam, there comes: the transmittance $T$ is

$$
T=\frac{P_{t}}{P_{i n c}}=\frac{\varepsilon_{2}}{\varepsilon_{1}} \frac{\cos \left(\theta_{2}\right)}{\cos \left(\theta_{1}\right)} \frac{n_{1}}{n_{2}} t^{2}=\frac{\tan \left(\theta_{1}\right)}{\tan \left(\theta_{2}\right)} t^{2}
$$

taking into account that $\varepsilon=n^{2}$

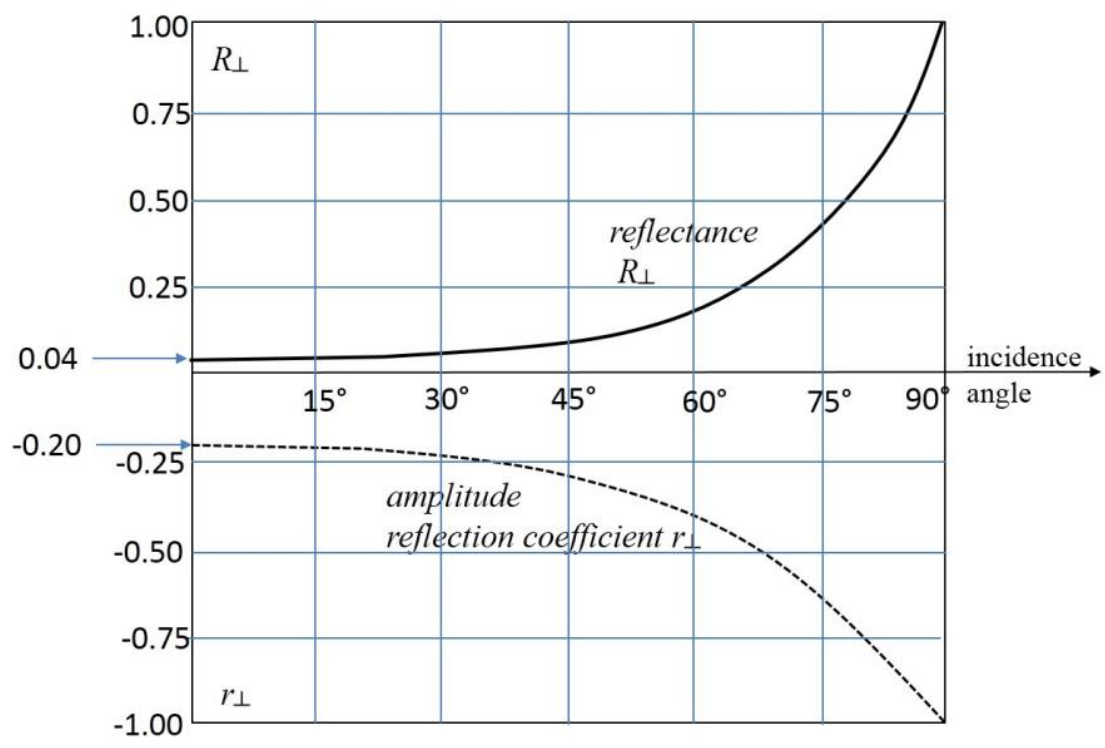


Figure 1.3: Amplitude reflection coefficient $r \perp$ and reflectance $R_{\perp}$ versus incidence angle, from air to glass of refractive index $n=1.5$ (TE wave).

\subsubsection{External and internal reflections}

\subsubsection{External reflection}

External reflection is said to occur when $n_{1}<n_{2}$, for example when light propagates from air into glass, or from air into water. Here again, we will first consider the case of polarized light, with the $\boldsymbol{E}$ vector being either perpendicular or parallel to the plane of incidence, then the case of natural light and finally the case of randomly polarized light.

- $\quad \boldsymbol{E}$ vector perpendicular to the plane of incidence:

Variations of $r_{\perp}$ with respect to angle of incidence $\theta_{1}$ are given on the graph of figure 1.3 in the case of an interface of air with a glass of refractive index 1.5: $r_{\perp}$ starts at -0.2 for normal incidence and reaches -1 for grazing incidence $\left(\theta_{1}=\pi / 2\right)$. Corresponding values of $R_{\perp}$ evolve from $4 \%$ up to $100 \%$.

\section{- $\quad \boldsymbol{E}$ vector parallel to the plane of incidence:}

$r$ // starts from the same value of -0.2 , as above for normal incidence, and changes in sign while crossing the zero value when $\theta_{1}+\theta_{2}=\pi / 2$, i.e. when reflected and refracted beams are perpendicular to each other. In that configuration, $\sin \left(\theta_{2}\right)=\cos \left(\theta_{1}\right)$, hence $n_{1} \cdot \sin \left(\theta_{1}\right)=n_{2} \cdot \cos \left(\theta_{1}\right)$ and consequently:

$$
\tan \left(\theta_{1}\right)=\frac{n_{2}}{n_{1}}(1.62)
$$

This particular angle of incidence for which the electric vector is parallel to the plane of incidence is not reflected at all is called Brewster's angle. It has a value of about $56^{\circ} 20^{\prime}$ for a glass of refractive index 1.50 . Reflectance decreases from $4 \%$ at normal incidence down to zero at Brewster's angle, then increases sharply up to $100 \%$ for grazing incidence, as shown on figure 1.4 . 


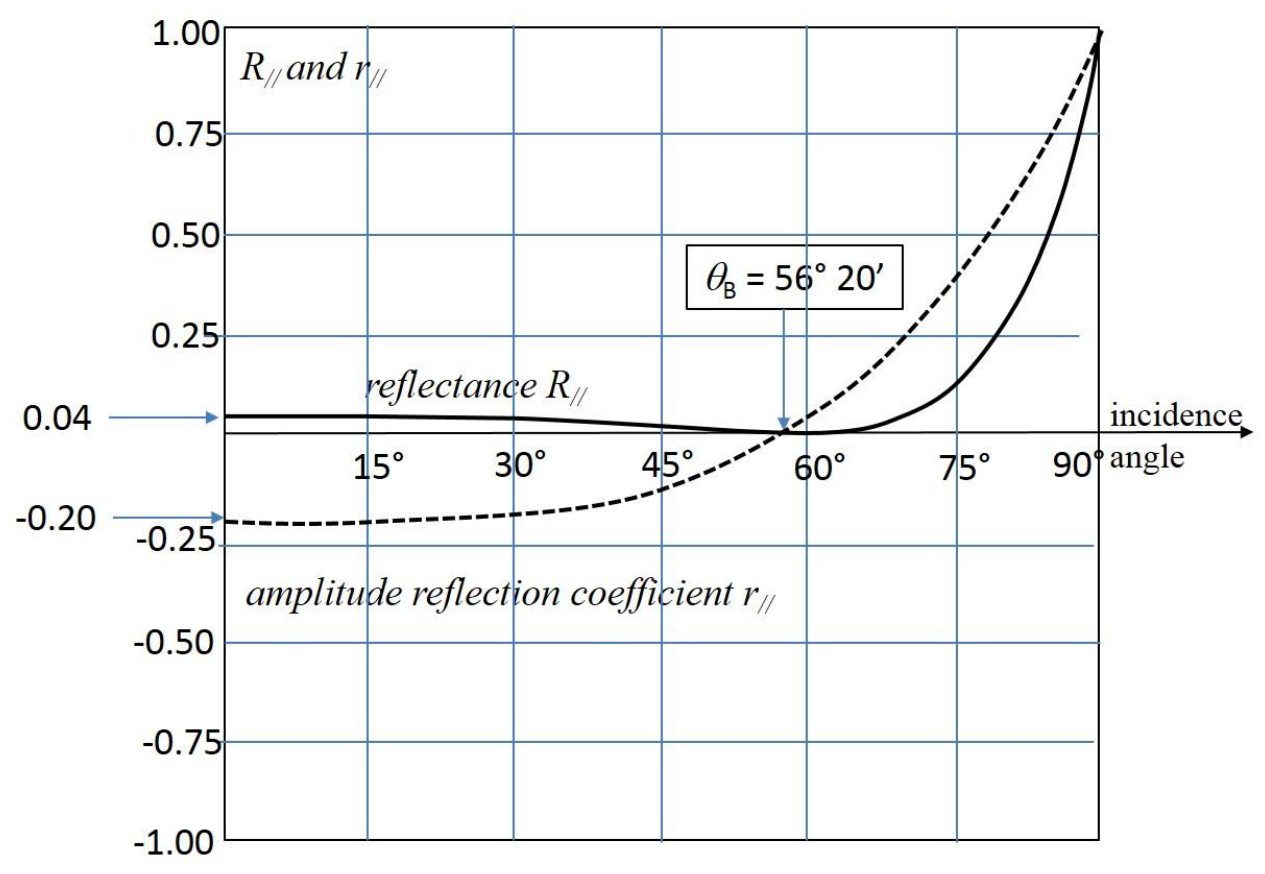

Figure 1.4: Amplitude reflection coefficient $r_{/ /}$and reflectance $R_{/ /}$versus incidence angle, from air to glass of index $n=1.5$ (TM wave; $\theta_{\mathrm{B}}$ corresponds to Brewster angle).

\section{- Case of natural light:}

The light being radiated from most natural sources is un-polarized, i.e. made up of a superposition of electric vectors vibrating along all possible directions. Such a light may be considered as having two perpendicular components $E_{/ /}$and $E_{\perp}$ of equal amplitude but however without any phase correlation. The reflectance for each of these components will be either $R_{/ /}$or $R_{\perp}$ and generally, reflected light will be partially polarized: it will be completely depolarized for $\theta_{1}=0$ and $\theta_{1}=\pi / 2$ and totally polarized (perpendicular to the plane of incidence) for $\theta_{1}=\theta_{\mathrm{B}}$.

- Case of light linearly polarized along some arbitrary direction:

If the electric vector $\boldsymbol{E}$ is at angle $\xi$ with the plane of incidence (figure 1.2a), it can be decomposed into two in phase components:

$$
E_{/ /}=E \cos (\xi) \text { and } E_{\perp}=E \sin (\xi) \text { (1.63) }
$$

After reflection, one will get:

$$
E^{\prime}{ }_{/ /}=r_{/ /} E \cos \left(\xi^{\prime}\right) \text { and } E_{\perp}^{\prime}=r_{\perp} E \cos \left(\xi^{\prime}\right)
$$

Since these two components are in phase, the reflected vibration will still be linearly polarized, at an angle $\xi^{\prime}$ with the plane of incidence such that: 


$$
\tan \left(\xi^{\prime}\right)=\frac{E_{\perp}^{\prime}}{E_{\prime /}^{\prime}}=\tan \left(\frac{r_{\perp}}{r_{/ /}}\right)=\frac{\cos \left(\theta_{1}-\theta_{2}\right)}{\cos \left(\theta_{1}+\theta_{2}\right)} \tan (\xi)
$$

Since the coefficient of $\tan (\xi)$ is always $>1$, the plane of vibration tends to get away from the plane of incidence.

\subsubsection{Internal reflection}

Internal reflection is said to occur when $n_{1}>n_{2}$, which may arise for instance when a beam propagating inside a piece of glass hits the glass/air boundary. Equations (1.55), (1.58), (1.60) and (1.61) which give the characteristic parameters of the reflected and transmitted waves still hold, provided that $\theta_{1}$ is changed into $\theta_{2}$ and vice versa. Three cases may thus occur:

- As long as $\theta_{1}$ remains smaller than a so-called "limit angle" $\theta_{L}$ satisfying the relationship:

$$
\sin \left(\theta_{L}\right)=\frac{n_{2}}{n_{1}}(1.66)
$$

the refraction angle $\theta_{2}$ does exist and part of the light gets transmitted towards the outside (external) medium

- For $\theta_{1}=\theta_{L}, \theta_{2}=90^{\circ}$ and the emerging beam appears as grazing along the interface figuring the boundary between both the internal and external media.

- For an incidence angle $\theta_{1}$ greater than $\theta_{\mathrm{L}}$, the angle of refraction $\theta_{2}$ does not exist anymore, but one may nevertheless introduce "virtual" values of $\sin \left(\theta_{2}\right)>1$ and pure imaginary ones for $\cos \left(\theta_{2}\right)$ in above mentioned expressions. By writing now:

$$
n_{2,1}=\frac{n_{2}}{n_{1}}(1.67)
$$

one gets:

$$
\begin{gathered}
\sin \left(\theta_{2}\right)=\frac{\sin \left(\theta_{1}\right)}{n_{2,1}} \text { and } \cos \left(\theta_{2}\right) \text { becomes purely imaginary: } \cos \left(\theta_{2}\right)= \pm i \sqrt{\frac{\sin ^{2}\left(\theta_{1}\right)}{n_{2,1}^{2}}-1}= \pm i m \\
\text { by setting } m=\sqrt{\frac{\sin ^{2}\left(\theta_{1}\right)}{n_{2,1}^{2}}-1} \text { (1.68). }
\end{gathered}
$$

This leads to the fact that $\sin \left(\theta_{1}-\theta_{2}\right)$ and $\sin \left(\theta_{1}+\theta_{2}\right)$ are complex conjugates, as well as $\tan \left(\theta_{1}-\theta_{2}\right)$ and $\tan \left(\theta_{1}+\theta_{2}\right)$. Therefore both moduli of $r_{/ /}$and $r_{\perp}$ equal unity and the corresponding incident energies are totally reflected: 


$$
R_{/ /}=R_{\perp}=100 \%
$$

Figure 1.5 shows reflectance values $R_{/ /}$and $R_{\perp}$ versus the angle of incidence $\theta_{1}$ in the case of a glass to air internal reflection $\left(n_{1}=1.5 ; n_{2}=1.0\right)$.

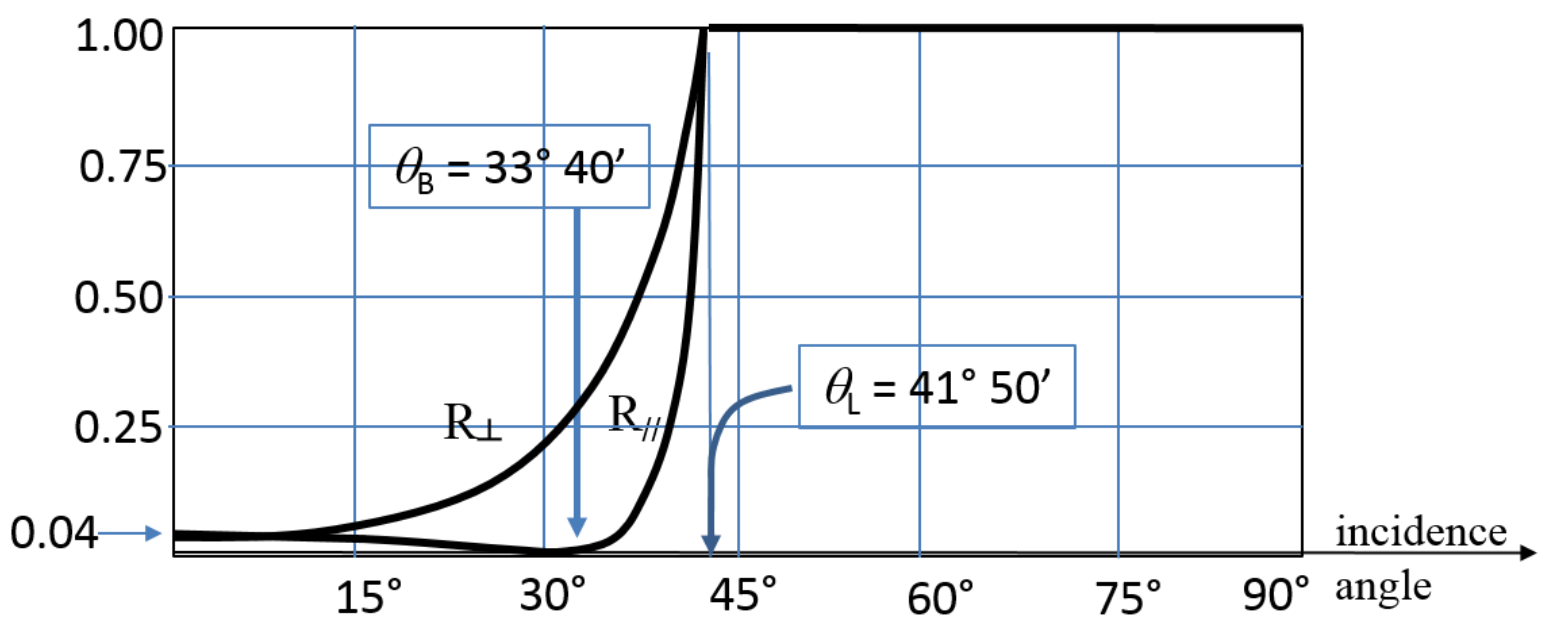

Figure 1.5: Reflectance $\left(R_{\perp}\right.$ and $R_{\|}$) of a glass to air interface versus incidence angle; $\theta_{\mathrm{B}}$ and $\theta_{\mathrm{L}}$ represent the Brewster and limit angles, respectively.

An interesting feature of the case of total internal reflection is the occurrence of a so-called evanescent wave in the external medium: when applying equations 1.49 and 1.50 to this case $\left(\theta_{1}>\theta_{L}\right)$, one gets the following expression for the transmitted field:

$$
\begin{gathered}
E_{t}=t E_{0} \exp \left[i k_{2}\left(\alpha_{2} x+\gamma_{2} z\right)\right] \text { (1.70), } \\
\text { with } \alpha_{2}=\sin \left(\theta_{2}\right)(1.71) \text { and } \gamma_{2}=\cos \left(\theta_{2}\right)= \pm i m \text { (1.72) }
\end{gathered}
$$

Taking into account that $k_{2} \alpha_{2}=k_{1} \alpha_{1}=k_{1} \sin \theta_{1}$ and that $i k_{2} \gamma_{2} z$ becomes a real quantity, one may write:

$$
E_{t}=t E_{0} e^{-k_{2} m z} \exp \left[i k_{1} x \sin \left(\theta_{1}\right)\right]
$$

One will remark that the minus sign has to be kept in the expression of $\cos \left(\theta_{2}\right)$ in equation 1.72 to avoid the propagation of an infinitely increasing energy in the output medium. As a consequence, the transmitted wave "creeps" along the boundary while, in the mean time, its amplitude decreases exponentially along the $z$ axis which explains why it is called an "evanescent wave".

We can also analyze the phase shift arising in the case of total internal reflection, depending on the polarization state of the beam: 
a) In the case of an incident TE wave, which is the component perpendicular $\left(E_{\perp}\right)$ to the plane of incidence, the amplitude reflection coefficient may be written as:

$$
r_{\perp}=-\frac{\sin \left(\theta_{1}-\theta_{2}\right)}{\sin \left(\theta_{1}+\theta_{2}\right)}=\frac{\sin \left(\theta_{2}\right) \cos \left(\theta_{1}\right)+i m \sin \left(\theta_{1}\right)}{\sin \left(\theta_{2}\right) \cos \left(\theta_{1}\right)-i m \sin \left(\theta_{1}\right)}=\exp \left(i \varphi_{\perp}\right)
$$

and the phase shift $\varphi_{\perp}$ at reflection is given by:

$$
\tan \left(\frac{\varphi_{\perp}}{2}\right)=\frac{\sqrt{\sin ^{2}\left(\theta_{1}\right)-n_{2,1}^{2}}}{\cos \left(\theta_{1}\right)}(1.75)
$$

Thus $\varphi_{\perp}$ is an advance phase shift that increases from 0 for $\theta_{1}=\theta_{L}$ up to $\pi$ for $\theta_{1}=\frac{\pi}{2}$

b) In the case of an incident TM wave, which is the component parallel $\left(E_{/ /}\right)$to the plane of incidence), one gets:

$$
r_{/ /}=\frac{\tan \left(\theta_{2}-\theta_{1}\right)}{\tan \left(\theta_{1}+\theta_{2}\right)}=-\frac{\sin \left(\theta_{1}\right) \cos \left(\theta_{1}\right)+i m \sin \left(\theta_{2}\right)}{\sin \left(\theta_{1}\right) \cos \left(\theta_{1}\right)-i m \sin \left(\theta_{2}\right)}=\exp \left(i \varphi_{/ /}\right)
$$

As in the case of a TE wave, there is also an advance phase shift at reflection, such that:

$$
\tan \left(\frac{\varphi_{I I}}{2}\right)=\frac{\sqrt{\sin ^{2}\left(\theta_{1}\right)-n_{2,1}^{2}}}{n_{2,1}{ }^{2} \cos \left(\theta_{1}\right)}(1.77)
$$

This phase shift starts at $O$ for $\theta_{1}=\theta_{L}$ and increases up to $\pi$ for $\theta_{1}=\frac{\pi}{2}$. Figure 1.6 shows the difference: $\left(\varphi_{/ /}-\varphi_{\perp}\right)$ versus the angle of incidence, in the total reflection regime between glass $(n=1.5)$ and air.

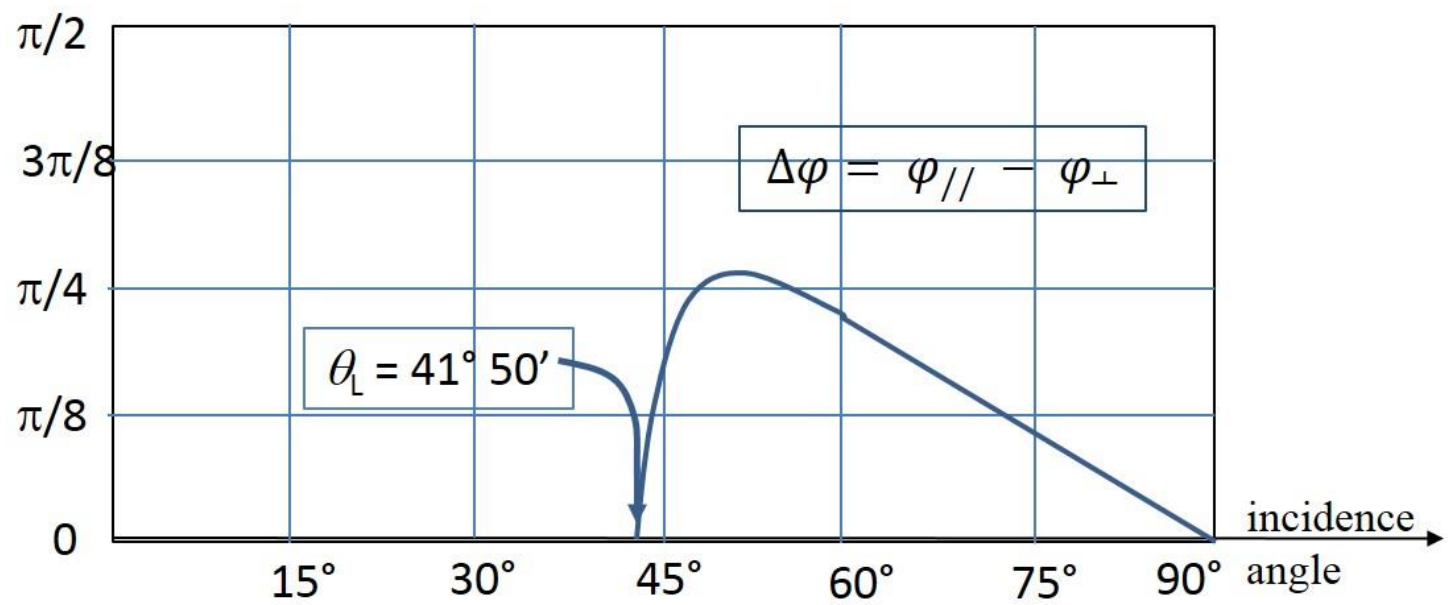

Figure 1.6: Phase shift difference in the total reflection regime for a glass $(n=1.5)$ to air interface, versus incidence angle. 


\subsubsection{Case of thin films}

\subsubsection{Transfer matrix formulation}

A simple extension of the above analysis occurs in the case of a thin, plane parallel film of material covering the surface of a substrate $[7,8]$. The presence of two (or more) interfaces means that a number of beams will be produced by successive reflections and the properties of the film will be determined by the summations of these beams.

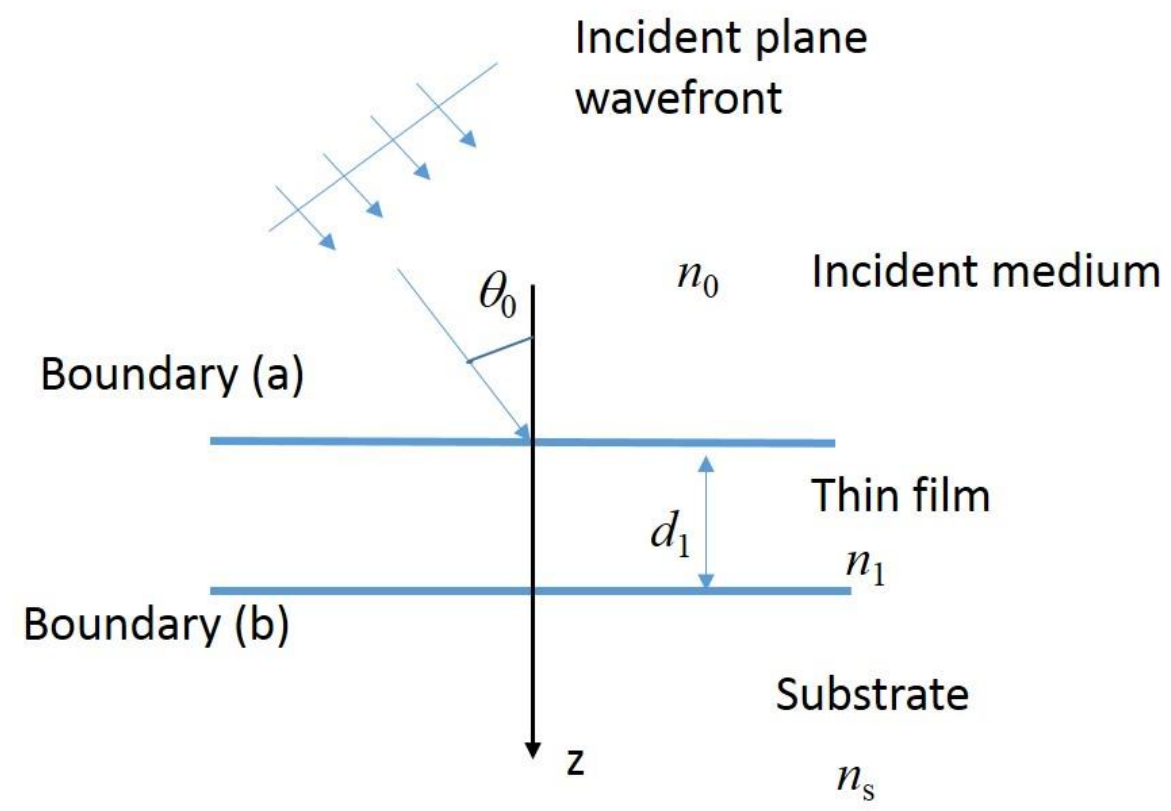

Figure 1.7: Plane wave incident on a thin film

We denote the waves propagating along the direction of incidence and those propagating towards the opposite direction by the symbols + and -, respectively. The interface (b) located between the film and the substrate can be treated in the same way as for a simple boundary. By considering the tangential components of the fields, there is no wave travelling within the substrate and all + waves can be summed.

Thus, at the substrate-thin film interface (b), the tangential components of $\boldsymbol{E}$ and $\boldsymbol{H}$ are:

$$
\left\{\begin{array}{c}
E_{b}=E_{1 b}^{+}+E_{1 b}^{-} \\
H_{b}=Y_{1} E_{1 b}^{+}-Y_{1} E_{1 b}^{-}
\end{array}\right.
$$

where $Y_{1}$ is the optical admittance defined as the ratio of the tangential components 


$$
\left\{\begin{array}{c}
E_{1 b}^{+}=\frac{1}{2}\left(\frac{H_{b}}{Y_{1}}+E_{b}\right) \\
E_{1 b}^{-}=\frac{1}{2}\left(-\frac{H_{b}}{Y_{1}}+E_{b}\right) \\
H_{1 b}^{+}=Y_{1} E_{1 b}^{+}=\frac{1}{2}\left(H_{b}+Y_{1} E_{b}\right) \\
H_{1 b}^{-}=-Y_{1} E_{1 b}^{-}=\frac{1}{2}\left(H_{b}-Y_{1} E_{b}\right)
\end{array}\right.
$$

The field at the other interface (a) is obtained by taking into account the phase change $\varphi$ (or phase shift) of the wave

$$
\varphi=\frac{2 \pi}{\lambda} n_{1} d_{1} \cos \left(\theta_{1}\right)
$$

where $d_{1}$ is the thickness of the layer

Note that $\theta_{1}$ is obtained by applying the Snell-Descartes relationship (1.52): $n_{0} \sin \left(\theta_{0}\right)=n_{1} \sin \left(\theta_{1}\right)$

$$
\left\{\begin{array}{c}
E_{1 a}^{+}=E_{1 b}^{+} e^{i \varphi} \\
E_{1 a}^{-}=E_{1 b}^{-} e^{-i \varphi} \\
H_{1 a}^{+}=H_{1 b}^{+} e^{i \varphi} \\
H_{1 a}^{-}=H_{1 b}^{-} e^{-i \varphi}
\end{array}\right.
$$

So that

$$
\left\{\begin{array}{c}
E_{a}=E_{1 a}^{+}+E_{1 a}^{-} \\
H_{a}=H_{1 a}^{+}+H_{1 a}^{-}
\end{array}\right.
$$

These relations can be written in a matrix form such as:

$$
\left[\begin{array}{c}
E_{a} \\
H_{a}
\end{array}\right]=\left[\begin{array}{cc}
\cos \varphi & \frac{i}{Y_{1}} \sin \varphi \\
i Y_{1} \sin \varphi & \cos \varphi
\end{array}\right]\left[\begin{array}{l}
E_{b} \\
H_{b}
\end{array}\right] \text { (1.83), }
$$

where the $2 \times 2$ matrix is characteristic of a homogeneous thin film and often called its Abeles matrix.

\subsubsection{Optical properties of a coating}

The procedure described in the preceding section can be extended to the general case of a $\mathrm{N}$ layers stack, where the resulting characteristic matrix is simply the product of the successive individual matrices corresponding to the sequence performed during the coating process used.

Starting from the substrate one can take into account step by step each one of the various appearing interfaces through the product of the corresponding matrices to connect finally the field tangential components $\left(E_{A}, H_{A}\right)$ and $\left(E_{s}, H_{s}\right)$ drawn on figure 1.8: 


$$
\left[\begin{array}{c}
E_{A} \\
H_{A}
\end{array}\right]=\prod_{j=1}^{N}\left[\begin{array}{cc}
\cos \varphi_{j} & i \frac{\sin \varphi_{j}}{Y_{j}} \\
i Y_{j} \sin \varphi_{j} & \cos \varphi_{j}
\end{array}\right] \cdot\left[\begin{array}{c}
E_{s} \\
H_{s}
\end{array}\right]
$$

where the optical phase change $\varphi_{j}$ introduced by the $\mathrm{j}^{\text {th }}$ layer) is defined by the expression:

$$
\begin{aligned}
& \varphi_{j}=\frac{2 \pi}{\lambda}\left(n_{j}-i \kappa_{j}\right) d_{j} \cos \left(\theta_{j}\right) \\
& \text { with } n_{j} \text { : refractive index of the } j^{\text {th }} \text { layer; } \\
& \kappa_{j} \text { : extinction coefficient of the }{ }^{\text {th }} \text { layer; } \\
& d_{j} \text { : mechanical thickness of the } j^{\text {th }} \text { layer; } \\
& \lambda \text { the wavelength and } \\
& Y_{j}=\left(n_{j}-i \kappa_{j}\right) / \cos \left(\theta_{j}\right) \text { for p-polarization (TM-wave or //) } \\
& Y_{j}=\left(n_{j}-i \kappa_{j}\right) \cos \left(\theta_{j}\right) \text { for s-polarization (TE-wave or } \perp \text { ) } \\
& Y_{s}=\left(n_{s}-i \kappa_{s}\right) / \cos \left(\theta_{s}\right) \text { for p-polarization } \\
& Y_{s}=\left(n_{s}-i \kappa_{s}\right) \cos \left(\theta_{s}\right) \text { for s-polarization } \\
& \text { and for the incident medium }\left(\mathrm{n}_{0}\right) \text { : } \\
& Y_{0}=n_{0} / \cos \left(\theta_{0}\right) \text { for p-polarization } \\
& Y_{0}=n_{0} \cos \left(\theta_{0}\right) \text { fors-polarization }
\end{aligned}
$$

Then speaking in terms of admittances, we get for a coating of N-layers [7-8]:

$$
\left[\begin{array}{l}
B \\
C
\end{array}\right]=\left(\prod_{j=1}^{N}\left[\begin{array}{cc}
\cos \varphi_{j} & i \frac{\sin \varphi_{j}}{Y_{j}} \\
i Y_{j} \sin \varphi_{j} & \cos \varphi_{j}
\end{array}\right]\right) \cdot\left[\begin{array}{c}
1 \\
Y_{s}
\end{array}\right]
$$

the reflectance at an interface (a) is $R=|r|^{2}=\left|\frac{Y_{0} B-C}{Y_{0} B+C}\right|^{2}=\left|\frac{Y_{0}-Y_{A}}{Y_{0}+Y_{A}}\right|^{2}$

$$
\text { the transmittance is } T=\frac{4 Y_{0} \operatorname{Re}\left(Y_{s}\right)}{\left|Y_{0} B+C\right|^{2}}
$$

where $\operatorname{Re}\left(Y_{s}\right)$ is the real part of $Y_{s}$

and the absorptance is $A=1-R-T$ (1.89) 


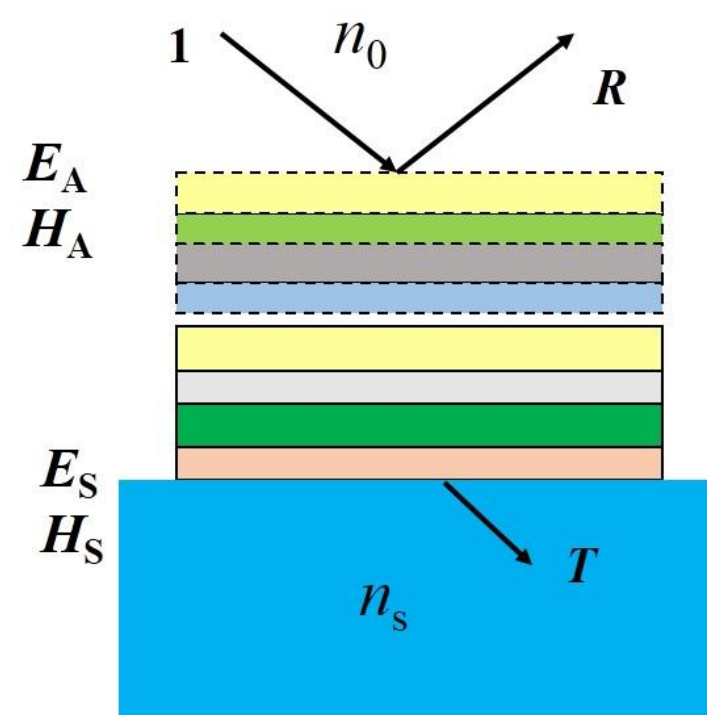

Figure 1.8: Diagram showing a multilayer deposited on a substrate considered as infinite. $E_{A}$ and $H_{A}$ represent the tangential components of fields at the upper interface. $E_{s}$ and $H_{s}$ are the tangential components of the field at the coating/substrate interface. $n_{s}$ and $n_{0}$ are respectively the refractive indices of the substrate and the input medium.

\subsection{Measurement of the refractive index of bulk materials}

The methods and set-ups used for measuring the refractive indices of bulk materials are based upon the index induced phenomena described in paragraph 1.1.3, mainly angular deviation of light by refraction through prisms, and also, to a lesser degree, total internal reflection including critical angle, and in some cases Brewster angle measurements.

\subsubsection{Minimum of deviation angle through a prism}

This method is recognized as the most accurate one for measuring the refractive index of bulk materials. It consists in illuminating a prism of known apex angle $\alpha$ by a monochromatic collimated beam of wavelength $\lambda$, and in measuring the minimum value $\delta_{m}$ of the deviation angle between input and output beams by means of a goniometer. Figure 1.9 shows a typical graph of deviation angle $\delta$ versus the angle of incidence $\theta$. Minimum deviation occurs when rays inside the prism are perpendicular to the bisector of apex angle, hence when input and output beams are symmetrical with respect to that bisector.

At wavelength $\lambda$ and temperature $T$, the refractive index of the prism material is then given by the following relationship:

$$
n(\lambda, T)=\frac{\sin \left[\left(\alpha(T)+\delta_{m}(\lambda, T)\right) / 2\right]}{\sin [\alpha(T) / 2]}
$$


The fact that the deviation angle is stationary in the neighborhood of this configuration somewhat alleviates precision constraints upon the setting of the corresponding incidence angle.

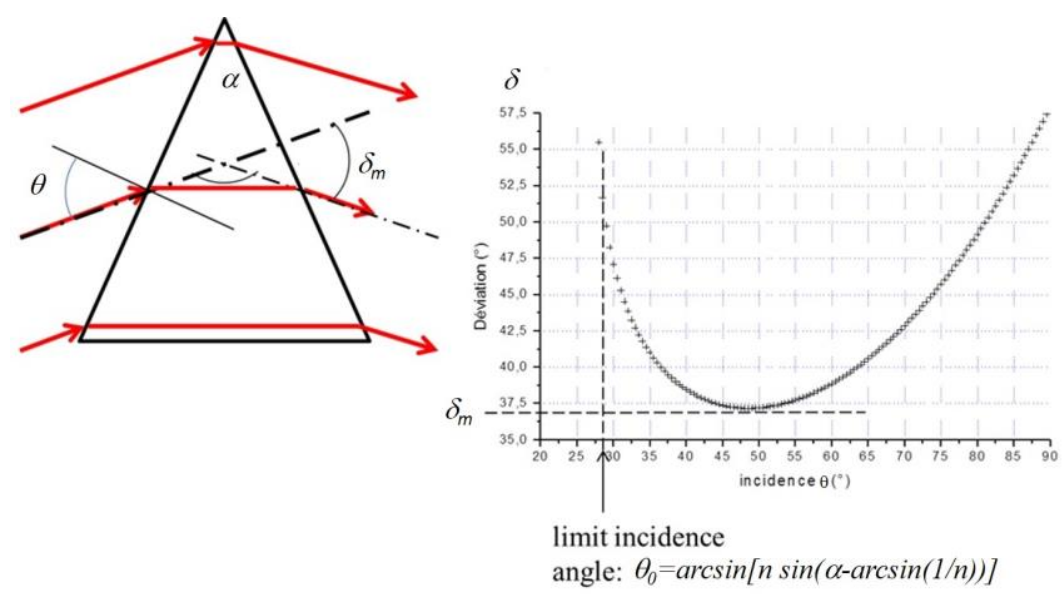

Figure 1.9: Deviation angle through a prism; minimum of deviation configuration

\section{Expected accuracy:}

The main experimental uncertainties $\sigma$ that are to be taken into account for evaluating the affordable accuracy on the measured value of $n(\lambda, T)$ are the following:

- $\sigma_{\alpha}$ upon the measurements of the apex angle of the prism, inducing a partial uncertainty $\sigma_{n, \alpha}=\left(\frac{\partial n}{\partial \alpha}\right) \sigma_{\alpha}$

- $\sigma_{\delta}$ upon the measurements of the deviation angle from the prism, inducing a partial uncertainty

$$
\sigma_{n, \delta}=\left(\frac{\partial n}{\partial \delta}\right) \sigma_{\delta}
$$

- $\sigma_{T}$ upon the measurements of the temperature $T$ of the prism, inducing $\sigma_{n, T}=\left(\frac{\partial n}{\partial T}\right) \sigma_{T}$ where $\left(\frac{\partial n}{\partial T}\right)$ is the value of the thermo-optic coefficient of the material, i.e. the change in its refractive index per degree Celsius.

- $\sigma_{\lambda}$ upon the measurements of the wavelength of the illuminating beam, inducing $\sigma_{n, \lambda}=\left(\frac{\partial n}{\partial \lambda}\right) \sigma_{\lambda}$ where $\left(\frac{\partial n}{\partial \lambda}\right)$ is the dispersive power of the material.

Considering that these uncertainties are uncorrelated, one may write that the global variance of the refractive index is the summation over all contributing variances:

$$
\sigma_{n}^{2}(\lambda, T)=\sigma_{n, \alpha}^{2}+\sigma_{n, \delta}^{2}+\sigma_{n, T}^{2}+\sigma_{n, \lambda}^{2}
$$


Even with commercial equipment, the minimum deviation method is quite satisfactory for most materials in the visible range. In the infrared, severe precautions must be taken concerning the size of the prisms, the precision of the angular measurements, the temperature of the room: firstly, the angular measurement accuracy degrades as wavelength increases from UV, visible up to infrared since it depends upon the (wavelength/pupil size) ratio and the signal to noise ratio; secondly, many infrared materials are semi-conductors, and their thermo-optic coefficient may be much larger than those of glasses of the visible domain (typically, one hundred times larger for germanium for instance).

Hence, in the IR domain, specific constraints are imposed upon the pupil size ( $>30 \mathrm{~mm})$, the surface quality (flatness better than $\lambda / 10$, usually measured in the visible), temperature, $\left(\sigma_{T}<0.1 \mathrm{~K}\right)$ and, in some cases, humidity and pressure.

A rough evaluation is given hereafter concerning the dependence of the index accuracy upon uncertainties on $\alpha$ and minimum deviation $\delta_{m}$ : taking the partial derivative of $n$ with respect to $\alpha$, one gets:

$$
\begin{gathered}
\frac{\partial n}{\partial \alpha}=\frac{\sin \left(\frac{\alpha}{2}\right) \cos \left(\frac{\alpha+\delta_{m}}{2}\right)-\sin \left(\frac{\alpha+\delta_{m}}{2}\right) \cos \left(\frac{\alpha}{2}\right)}{2 \sin ^{2}\left(\frac{\alpha}{2}\right)} \\
\frac{\partial n}{\partial \alpha}=\frac{\sqrt{1-\sin ^{2}\left(\frac{\alpha+\delta_{m}}{2}\right)}-n \cos \left(\frac{\alpha}{2}\right)}{2 \sin \left(\frac{\alpha}{2}\right)}
\end{gathered}
$$

Considering that the optimum value of apex angle $\alpha$ must be chosen in accordance with the expected value of $n$ ( $\alpha$ decreases as $n$ increases), and varies widely from one material to another, we choose to evaluate $\left(\frac{\partial n}{\partial \alpha}\right)$ with respect to $n$ and the angle of incidence, considered as being the same for all materials. Making use of some characteristics of the minimum deviation configuration:

If $\theta_{r}$ is the angle of the refraction,

$$
\begin{aligned}
\sin (\theta) & =n \sin \left(\theta_{r}\right) \text { and } \delta_{m}=2 \theta-\alpha \\
\alpha & =2 \arcsin \left(\frac{\sin (\theta)}{n}\right)(1.94)
\end{aligned}
$$

one gets:

$$
\frac{\partial n}{\partial \alpha}=\frac{n \cos (\theta)-n \sqrt{n^{2}-\sin ^{2}(\theta)}}{2 \sin (\theta)}
$$


Similarly, one gets the following result for $\frac{\partial n}{\partial \delta}$ where $\delta$ is deduced by measuring twice this deviation between two symmetrical positions of the prism with respect to the axis of the incident beam:

$$
\frac{\partial n}{\partial \delta}=\frac{n}{4 \tan (\theta)}
$$

Table 1.2 shows a comparative evaluation between the expected accuracy of the minimum deviation method for the measurement of refractive indices of various infrared materials: magnesium fluoride, zinc sulfide, zinc selenide, silicon and germanium. For this evaluation, the angle of incidence is the same for all samples $\left(\theta=50^{\circ}\right), \sigma_{\alpha}=1$ second of an arc, $\sigma_{\delta}=6$ seconds of an arc, $\sigma_{T}=0.1^{\circ} \mathrm{C}$ and $\sigma_{\lambda}=1$ $\mathrm{nm}, 2 \mathrm{~nm}$ and $5 \mathrm{~nm}$ respectively at 3 different wavelengths: $2.325 \mu \mathrm{m}, 3.39 \mu \mathrm{m}$ and $10.6 \mu \mathrm{m}$.

The results from this analysis indicate that the respective contributions of the various parameters vary widely from a sample to another and for a given sample from wavelength to wavelength. The table below lists these relative weights for the above mentioned materials:

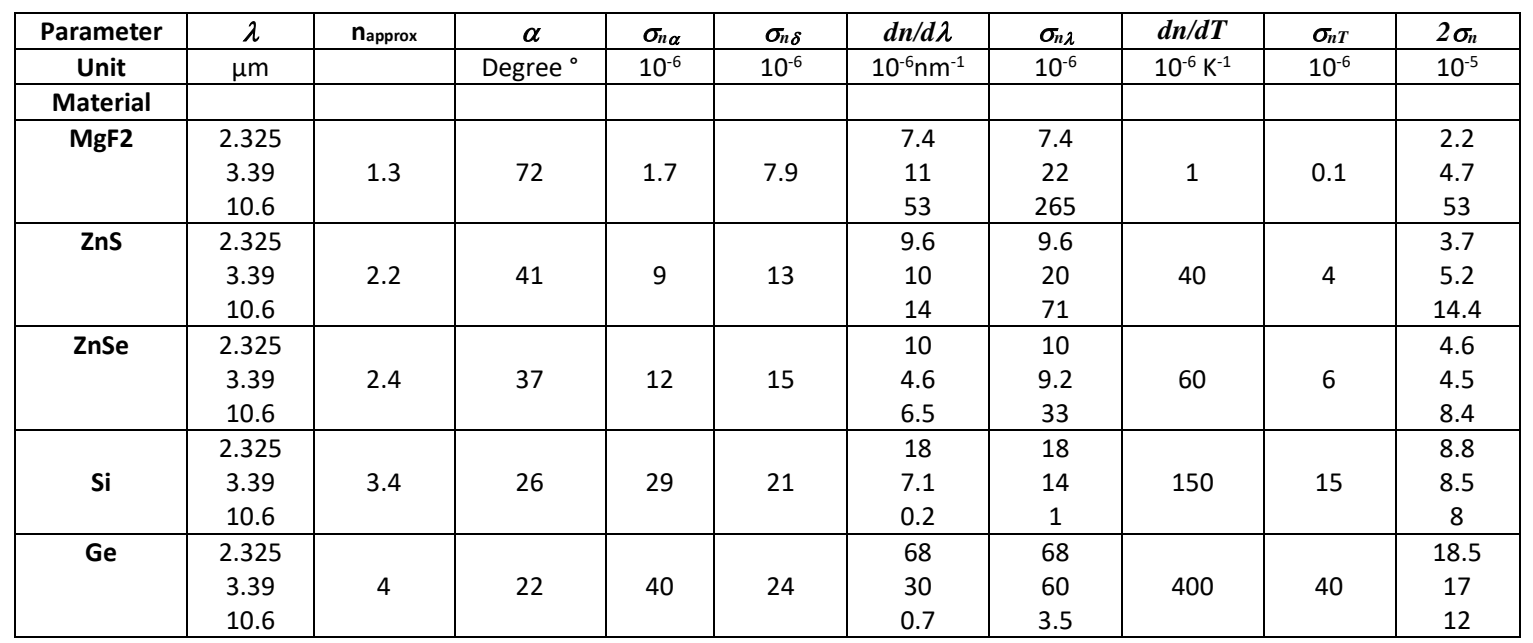

Table 1.2 : Contribution of the various sources of uncertainties on $\alpha, \delta$, $\lambda$ and $T$ to the index measurement accuracy by means of the minimum deviation method

\begin{tabular}{|c|c|c|c|c|c|c|c|}
\hline Parameter & $\lambda$ & $n_{\text {approx }}$ & $2 \sigma_{n}$ & $\sigma_{\alpha}=1$,' & $\sigma_{n \delta}=6$ "' & $\sigma_{\lambda}=1.2 \mathrm{~nm}$ & $\sigma_{T}=0.1 \mathrm{~K}$ \\
\hline Unit & $\mu \mathrm{m}$ & & $10^{-5}$ & $\%$ & $\%$ & $\%$ & $\%$ \\
\hline \multicolumn{8}{|l|}{ Material } \\
\hline \multirow{3}{*}{ MgF2 } & 2.325 & \multirow{3}{*}{1.3} & 2.2 & 3 & 51 & 46 & 0 \\
\hline & 3.39 & & 4.7 & 0.5 & 11 & 88 & 0 \\
\hline & 10.6 & & 53 & 0 & 0 & 100 & 0 \\
\hline \multirow{3}{*}{$\mathrm{ZnS}$} & 2.325 & \multirow{3}{*}{2.2} & 3.7 & 22 & 50 & 23 & 5 \\
\hline & 3.39 & & 5.2 & 12 & 25 & 60 & 2.5 \\
\hline & 10.6 & & 14.4 & 1.5 & 3 & 95 & 0 \\
\hline \multirow{3}{*}{ ZnSe } & 2.325 & \multirow{3}{*}{2.4} & 4.6 & 27 & 44 & 20 & 9 \\
\hline & 3.39 & & 4.5 & 28 & 45 & 18 & 9 \\
\hline & 10.6 & & 8.4 & 9 & 15 & 73 & 0 \\
\hline \multirow{3}{*}{ Si } & 2.325 & \multirow{3}{*}{3.4} & 8.8 & 46 & 24 & 18 & 12 \\
\hline & 3.39 & & 8.5 & 49 & 26 & 12 & 13 \\
\hline & 10.6 & & 8 & 55 & 30 & 0 & 15 \\
\hline \multirow{3}{*}{ Ge } & 2.325 & \multirow{3}{*}{4} & 18.5 & 18 & 6 & 58 & 18 \\
\hline & 3.39 & & 17 & 22.5 & 5 & 50 & 22.5 \\
\hline & 10.6 & & 12 & 42 & 16 & 0 & 16 \\
\hline
\end{tabular}


Table 1.3: Relative percentages of various uncertainty contributions on apex angle $\alpha$, deviation angle $\delta$, wavelength $\lambda$ and temperature $T$ to the overall refractive index uncertainty

Most national metrological laboratories such as PTB in Germany [9], LNE in France, or NPL in Great Britain are equipped with this method to measure refractive indices with respect to air, at least in the UV, visible and near infrared domains, for measurements at ambient temperature. One may mention the NIST and the NASA Goddard Space Flight Center that are able to measure absolute refractive indices down to cryogenic temperatures, up to the mid infrared [10-13].

\subsubsection{Littrow method}

Two other arrangements, derived from the minimum deviation method, have been set into operation. The first one, called front face autocollimation method (Figure 1.10a) consists in illuminating a prism under normal incidence upon the input face and measuring the corresponding deviation of the output beam. It has been widely used for the first characterizations of infrared materials, between 1950 and 1980 (Optical Science Center, Tucson, University of Arizona, Institut d'Optique, Paris). It has been replaced in several labs (Schott Glasswerke, Institut d'Optique) by the Littrow method (Figure 1.10b), in which the impinging beam hits the second face of the prism under normal incidence and is then retro-reflected by this optical face.

The configurations of these set-ups are shown schematically on Figure 1.10, with corresponding relationships between $\alpha, \delta$ and $n$; a typical set-up for the Littrow configuration is shown in Figure 1.11.

Analysis of these techniques shows that they are less accurate than the minimum deviation method, typically by respective factors of 2 (Littrow set-up) and 4 (auto-collimation on front face).

\subsubsection{Methods based upon grazing incidence and total internal reflection}

\section{Grazing incidence:}

Principle: when a ray propagating inside a medium of refractive index $n_{1}$ is launched under grazing incidence $\left(\theta_{1}=90^{\circ}\right)$ at the interface with a medium of index $n_{2}>n_{1}$, it is refracted inside the second medium along the direction of the limit angle $\theta_{1}$ such that:

$$
\sin \left(\theta_{L}\right)=\frac{n_{1}}{n_{2}}
$$

If an incident beam, comprising rays under grazing incidence, converges at some point I (Figu re 1.12) of such an interface, the beam refracted in the second medium disappears beyond the angle $\theta_{L}$ thus defining a well contrasted separation line between bothclear and dark areas. The second medium being 
a reference of precisely known refractive index, the measurement of the refractive index $n_{1}$ comes out from the measured value of the limiting direction $\theta_{L}$.
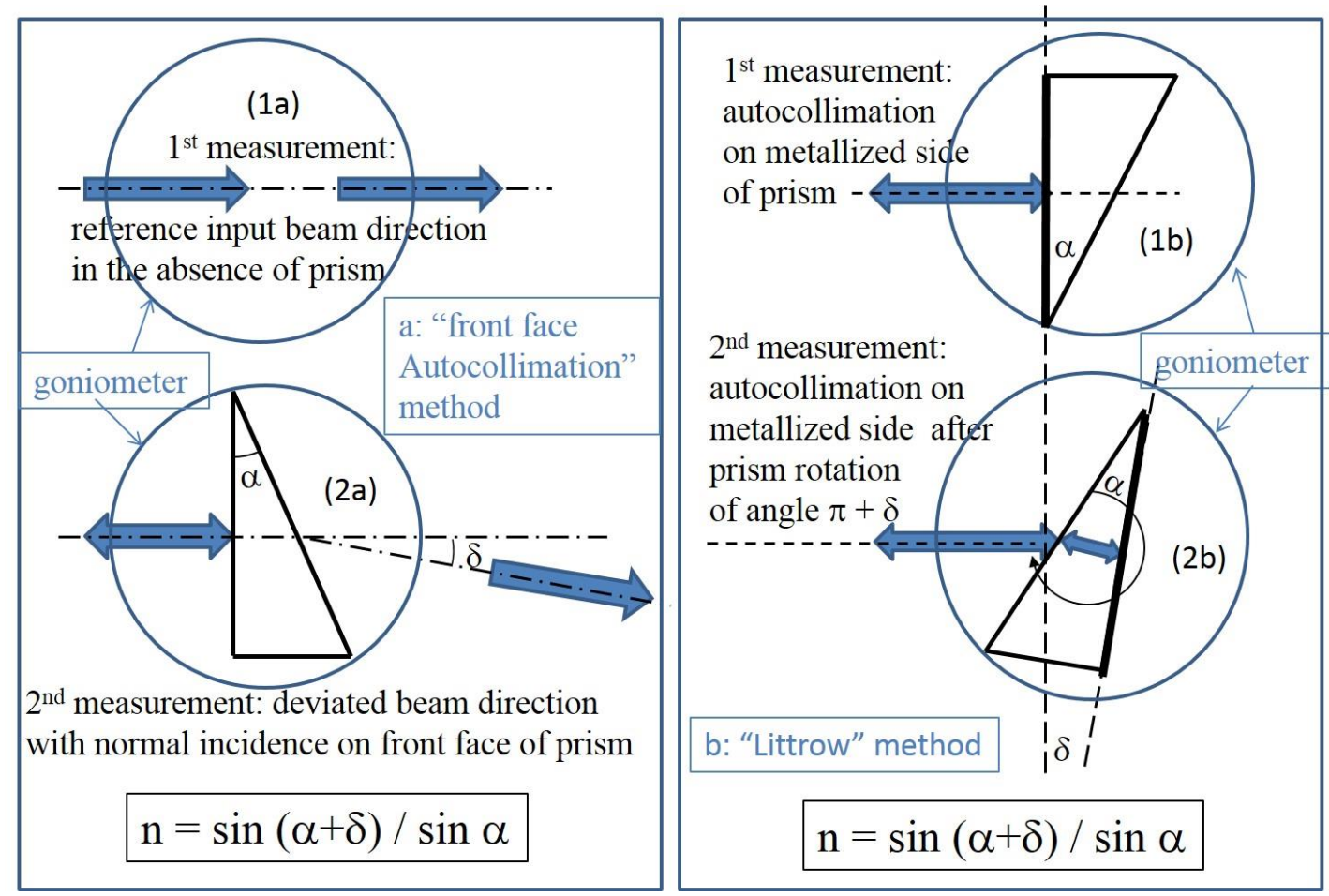

Figure 1. 10: "front face autocollimation" (a) and (b) "Littrow" configurations
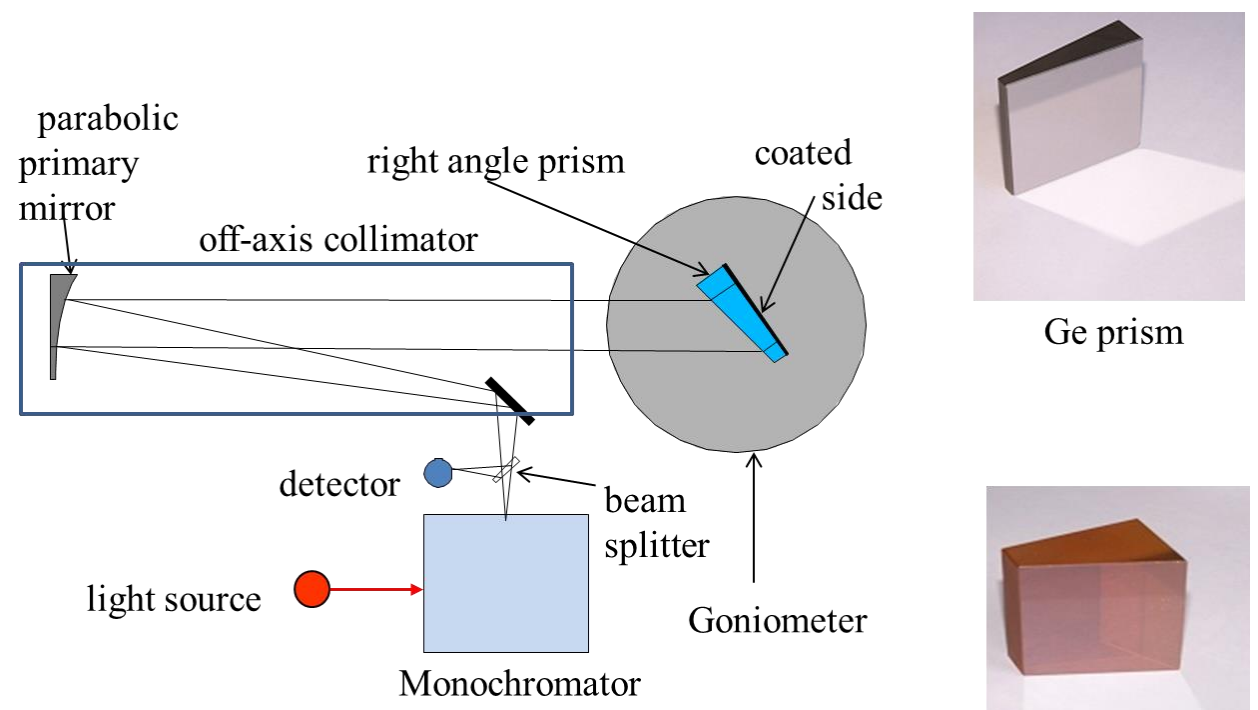

Ge prism

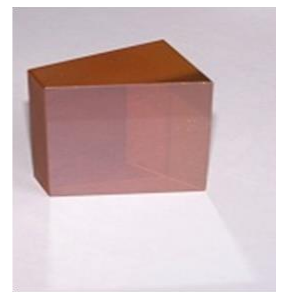

ZnSe prism

Figure 1.11: Typical experimental set-up and prisms for Littrow configuration 


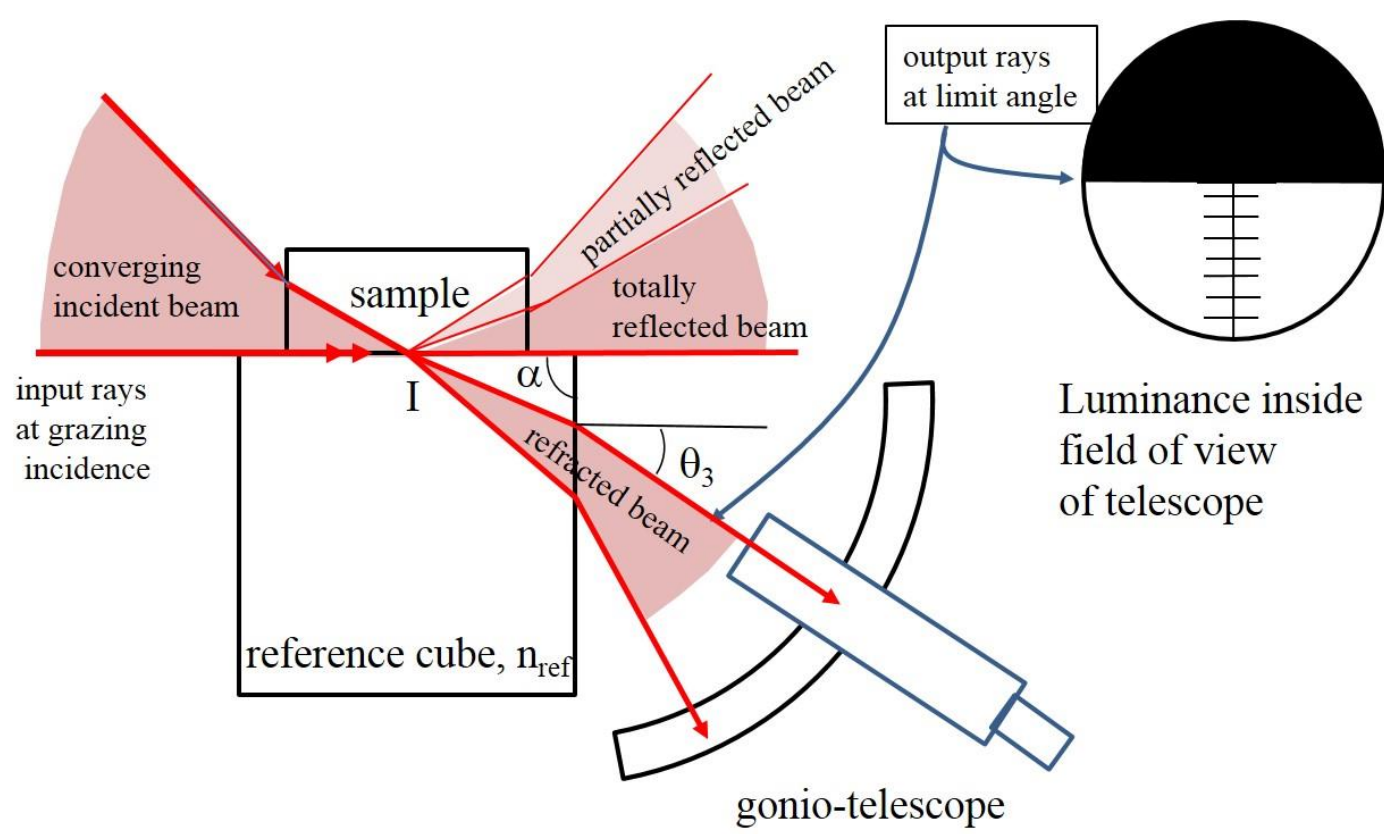

Figure 1.12: Configuration of Pulfrich refractometer

\section{Practical set-up: Pulfrich refractometer}

In a Pulfrich refractometer, the sample to be characterized is placed on top of the reference glass shaped as a cube, the horizontal and output faces of which being precisely polished and approximately normal to each other (angle $\alpha \approx 90^{\circ} \circ$ on Figure 1.12). In order to reduce stray light reflections from the interface, some immersion liquid index matched to the reference cube is also deposited in between the reference and the sample. The illuminating beam, containing rays that are parallel to the interface, is first refracted into the reference cube under the limit angle $\theta_{L}$ then refracted out of it under the angle of refraction $\theta_{3}$.

The refractive index of the sample, $n_{\text {sample }}$ may be obtained from $n_{\text {ref }}, \alpha$ and $\theta_{3}$ by the following relationship:

$$
\left.n_{\text {sample }}=\sin (\alpha) \sqrt{n_{\text {ref }}^{2}-\sin ^{2}\left(\theta_{3}\right.}\right)-\cos (\alpha) \sin \left(\theta_{3}\right) \text { (1.98), }
$$

where the angle $\theta_{3}$ is measured by means of the gonio-telescope also used as autocollimator, by pointing the normal to the output surface of the reference cube and the direction of the limit between dark and bright areas. In the visible domain, where the Pulfrich refractometer is exclusively used, the uncertainty that can be expected from it is of the order of $10^{-4}$.

\section{$\underline{\text { Total internal reflection }}$}

If a ray is incident from medium 1 , of index $n_{1}$ onto a medium 2 of index $n_{2}<n_{1}$, it is totally or partially reflected back towards the first medium if the angle of incidence is either greater or lower than the limit angle $\theta_{L}$, respectively. Hence, if the interface is illuminated by a beam 
converging at some point I (Figure 1.13) of the interface, there results by reflection back to the first medium, a separation line between a bright region and a dark one. This method may give rise to two different configurations; one of which does not necessitate that the illuminating light propagates through the sample. In this case, this method is of particular interest for measuring refractive indices of absorbing media that cannot be characterized by prism or grazing incidence methods.

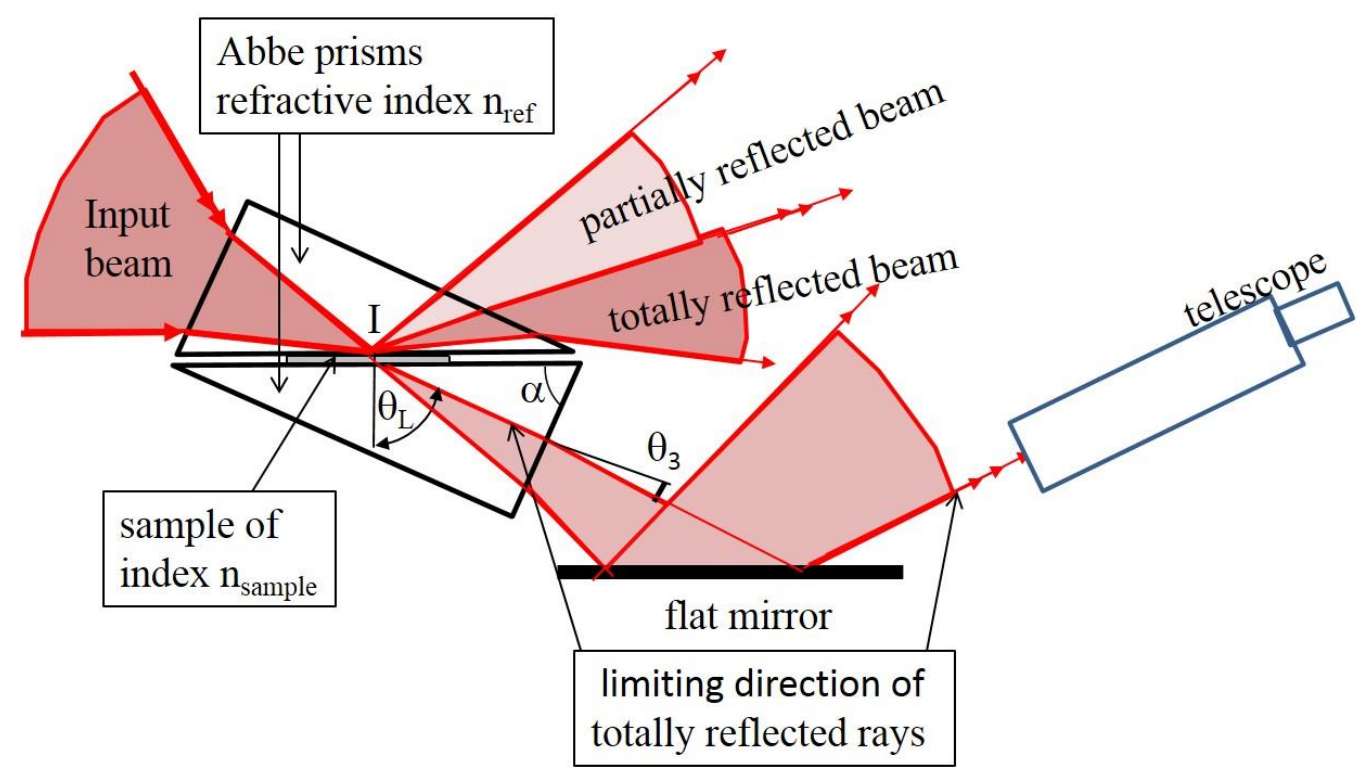

Figure 1.13: Abbe refractometer configuration for transparent media

The Abbe refractometer is meant essentially to measure the refractive index of liquids, viscous fluids, or gels. It is composed of two identical, reference prisms, one for illumination and the other one for measurement purposes. They are of known, high refractive index $\left(n_{\text {ref }}>1.7\right)$, and of standardized shape (right angle triangle, with angles $\alpha=60^{\circ}, \beta=90^{\circ}, \gamma=30^{\circ}$ ). The hypotenuse side of the measurement prism is set horizontal so that small amounts of liquids can be poured on top of it. If the liquid medium to be characterized is transparent, the illumination prism is deposited face to bottom on top of the measurement prism to spread the liquid uniformly in between the two prisms, so that the resulting layer behaves optically as a thin and parallel face plate (Figure 1.13).

The rays that propagate with an angle of incidence larger than the total internal reflection angle $\theta_{L}$ are totally reflected back into the upper prism, while the other ones are partially reflected, and partially refracted into the measurement prism below that angle $\theta_{L}$ :

$$
\sin \left(\theta_{L}\right)=\frac{n_{\text {sample }}}{n_{\text {ref }}}
$$

By adapting equation (1.98) to this configuration, one finds:

$$
2 n_{\text {sample }}=\sqrt{3\left(n_{r e f}^{2}-\sin ^{2}\left(\theta_{3}\right)\right)}-\sin \left(\theta_{3}\right)(1.100)
$$


One may notice that, in this configuration, the dark region in the output beam is perfectly black since no radiation is transmitted beyond the total reflection angle. Hence, the contrast between these two regions is quite good. If the liquid is opaque, the illuminating prism is of no use, and the sample is then illuminated from below, through the hypotenuse side of the measurement prism. In this case, the contrast is not as high as in the previous configuration, since the fraction of incident rays with incidence angles below the total reflection angle are partially reflected, but it is usually good enough to give access to the refractive index of opaque liquids, not measurable by other means. Abbe refractometers are extensively used in chemical or food industries.

\subsubsection{Brewster angle method}

In this method, the sample is shaped as a plane parallel face plate and illuminated by a collimated, linearly polarized beam, at the vicinity of the Brewster angle (Figure 1.14). If the electric vector of the incident beam is parallel to the plane of incidence, $R_{/ /}$drops down to a minimum value when the sample is rotated back and forth around this angle of incidence, $\theta_{1}=\theta_{\mathrm{B}}$, for which $n=\tan \left(\theta_{B}\right)$

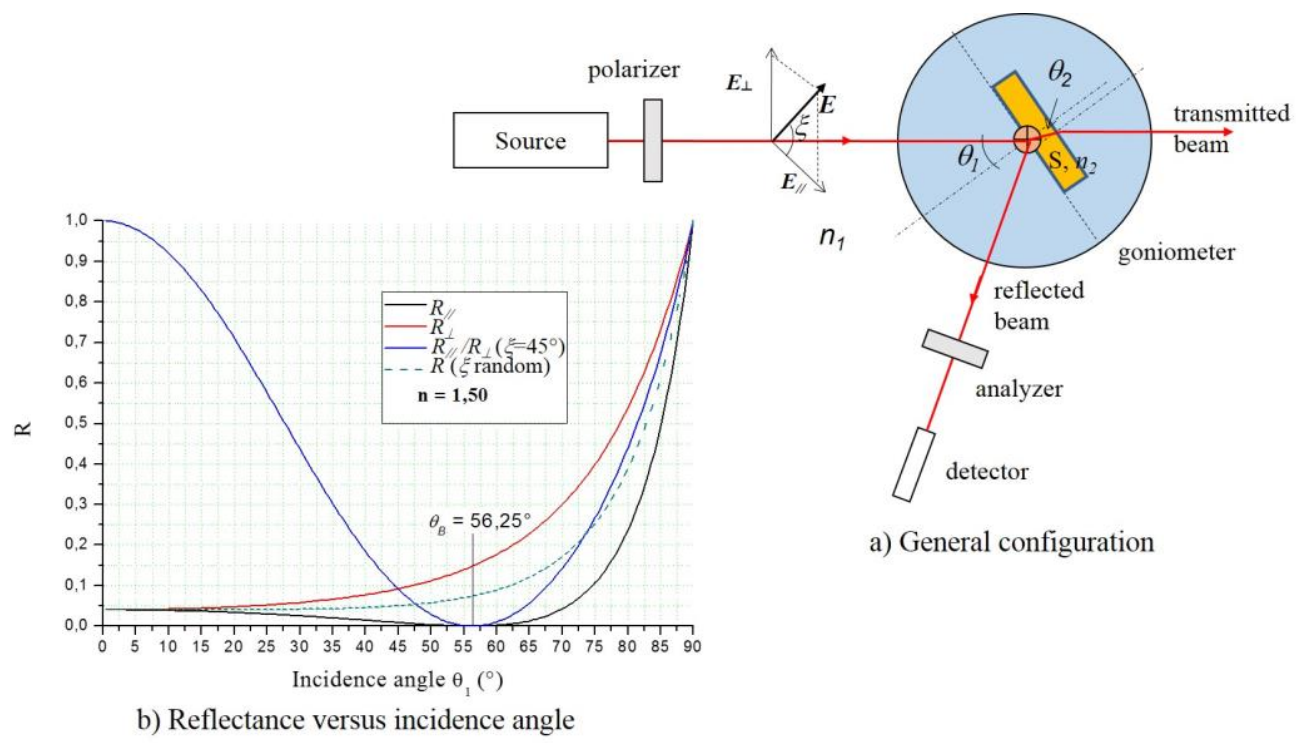

Figure 1.14: Brewster angle method: general configuration (a), and (b) reflectance versus incidence angle.

However the precision of this configuration is rather poor because the $R_{/ /}$curve is stationary around the Brewster angle of incidence. The accuracy may be somewhat improved by polarizing the input beam at $45^{\circ}$ with respect to the plane of incidence, and by recording the curves of both $R_{/ / /}$and $R_{\perp}$ with respect to the incident angle $\theta_{1}$ and solving for $n$ from the best fit between experimental and theoretical curves (Figure 1.14). A further improvement in accuracy may be obtained by applying a minor modulation e $d \theta_{1}$ around each angle of incidence and recording the resulting modulations $d R_{/ /}$ and $d R_{\perp}[14]$. As shown on Figure 1.15, the Brewster angle $\theta_{B}$ is then the value of the incidence angle for which there is a discontinuity of the first derivative of: 


$$
\frac{\partial \ln \left(\frac{R_{/ /}}{R_{\perp}}\right)}{\partial \theta_{1}}=0
$$

The uncertainty on refractive index of this method is of the order of $10^{-4}$.

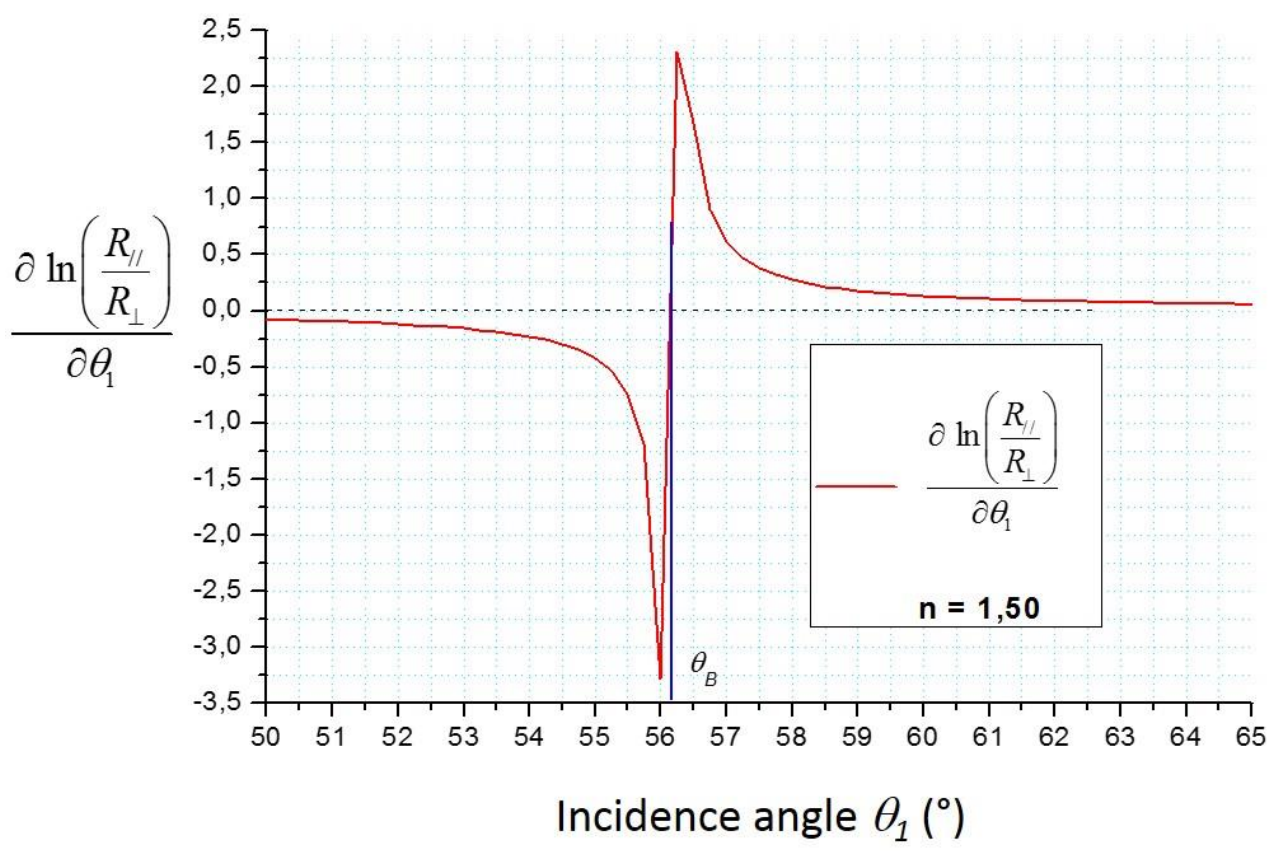

Figure 1.15: Possible improvement of refractive index measurement by Brewster angle determination

\subsubsection{Ellipsometric methods}

Ellipsometry is being widely used in refractive index measurements of bulk (or stacked) samples [1529]. It gives rise to a large number of methods and configurations that cannot be all covered here. That is why a selection has been made in this section that covers the operating principle and basic parameters of this technique, and is limited to the description of two classical configurations.

\subsubsection{Operating principle and measurement parameters}

Through the use of Fresnel formulae, equations (1.29) and (1.30) show how the refractive index $n(\omega)$ and extinction coefficient $\kappa(\omega)$ of a bulk material may be derived from spectroscopic measurements of the amplitude complex reflectance of the medium under normal incidence. In these equations, the phase change $\varphi(\omega)$ between reflected and incident waves at the boundary results from K-K relation (equation 1.28). 
In a more extensive use of Fresnel formulae, ellipsometry is based upon specific apparatuses ("ellipsometers") in which the sample (either bulk or stacked) is being illuminated under oblique incidence by a monochromatic beam of well controlled state of polarization. The refractive index of the sample is then computed from the measurement of the amplitudes and states of polarization of respectively the incident and reflected waves.

By expanding the amplitude reflection coefficient (equation 1.55) of an optical boundary, for the oblique incidence angle $\theta_{1}$ of a wave polarized perpendicularly to the incidence plane (TE polarization), one gets:

$$
r_{\perp}=\frac{\boldsymbol{E}_{T E, r}}{\boldsymbol{E}_{T E, i n c}}=-\left(\frac{\sin \left(\theta_{2}-\theta_{1}\right)}{\sin \left(\theta_{2}+\theta_{1}\right)}\right)=\left(\frac{n_{1} \cos \left(\theta_{1}\right)-\sqrt{n_{2}^{2}-n_{1}^{2} \sin ^{2}\left(\theta_{1}\right)}}{n_{1} \cos \left(\theta_{1}\right)+\sqrt{n_{2}^{2}-n_{1}^{2} \sin ^{2}\left(\theta_{1}\right)}}\right)
$$

and, similarly, for an incident linearly polarized wave parallel to the plane of incidence (TM polarization):

$$
r_{/ /}=\frac{\boldsymbol{E}_{T M, r}}{\boldsymbol{E}_{T M, i n c}}=-\left(\frac{\tan \left(\theta_{2}-\theta_{1}\right)}{\tan \left(\theta_{2}+\theta_{1}\right)}\right)=\left(\frac{-n_{2} \cos \left(\theta_{1}\right)+\frac{n_{1}}{n_{2}} \sqrt{n_{2}^{2}-n_{1}^{2} \sin ^{2}\left(\theta_{1}\right)}}{n_{2} \cos \left(\theta_{1}\right)+\frac{n_{1}}{n_{2}} \sqrt{n_{2}^{2}-n_{1}^{2} \sin ^{2}\left(\theta_{1}\right)}}\right)
$$

Hence, $r_{\perp}$ and $r_{/ /}$, which are real if both media are lossless $\left(\varphi \perp=\varphi_{/ /}=0\right.$ or $\left.\pi\right)$ and complex if either one of the media, or both, are absorbing $(\varphi \perp \neq \varphi / /)$, may be written as:

$$
r_{\perp}=\left|r_{\perp}\right| \exp \left(i \varphi_{\perp}\right) \text { and } r_{/ /}=\left|r_{/ /}\right| \exp \left(i \varphi_{/ /}\right)
$$

Let a monochromatic polarized beam of electric field $\boldsymbol{E}_{i n c}$ be incident upon the interface between two media of refractive indices $n_{1}$ and $n_{2}$ with the incidence angle $\theta_{1}$ :

$$
\boldsymbol{E}_{i n c}=\boldsymbol{E}_{T E} \cos \left(\omega t-\boldsymbol{k} \cdot \boldsymbol{r}+\varphi_{T E}\right)+\boldsymbol{E}_{T M} \cos \left(\omega t-\boldsymbol{k} \cdot \boldsymbol{r}+\varphi_{T M}\right)
$$

Where $\boldsymbol{E}_{T E}$ and $\boldsymbol{E}_{T M}$ are its components respectively perpendicular and parallel to the plane of incidence, with phases $\varphi_{T E}$ and $\varphi_{T M}$. The reflected electric field $\boldsymbol{E}_{r}$ is then:

$$
\boldsymbol{E}_{r}=\left|r_{\perp}\right| \boldsymbol{E}_{T E} \cos \left(\omega t-\boldsymbol{k} \cdot \boldsymbol{r}+\varphi_{T E}+\varphi_{\perp}\right)+\left|r_{/ /}\right| \boldsymbol{E}_{T M} \cos \left(\omega t-\boldsymbol{k} \cdot \boldsymbol{r}+\varphi_{T M}+\varphi_{/ /}\right)
$$

A comparison between equations (1.105) and (1.106), shows that, if the incident electric field is not perpendicular or parallel to the plane of incidence $\left(\left|\boldsymbol{E}_{T E}\right|\right.$ and $\left.\left|\boldsymbol{E}_{T M}\right| \neq 0\right)$, the states of polarization of the reflected and the incident beams differ from each other: for instance, if the input beam is plane polarized $\left(\varphi_{T E}=\mp \varphi_{T M}\right)$ and if the medium under test is non-absorbing $\left(n_{2}\right.$ real), the reflected beam 
is also plane polarized, but in a different direction, if the medium is non absorbing, and it is elliptically polarized if the medium is absorbing.

Since the angle of incidence $\theta_{1}$ and the refractive index $n_{1}$ of the first medium (usually air) are known parameters, the change in the state of polarization at reflection is a function of the unknown refractive index $n_{2}$ to be measured, and it is possible to deduce the refractive index $n_{2}$ by measuring $r_{\perp}$ and $r_{/ /}$. Since $n_{2}=n-i k$, there are two unknown, and two equations are necessary to fully characterize $n_{2}$.

In practice, ellipsometric techniques are devised to measure the following quantity, $\rho_{\square}$, the complex reflectance ratio, $r_{/ /}$over $r_{\perp}$ :

$$
\rho_{e}=\frac{r_{/ \prime}}{r_{\perp}}=\frac{\left|r_{/ \prime}\right|}{\left|r_{\perp}\right|} \exp \left[i\left(\varphi_{/ /}-\varphi_{\perp}\right)\right]=\tan \psi \exp (i \Delta)
$$

Where $\psi \in\left[0^{\circ}, 90^{\circ}\right]$ and $\Delta \epsilon\left[-180^{\circ},+180^{\circ}\right]$, the two basic parameters being measured, are such that $\tan \psi$ is the ratio between the (amplitude) attenuations of the TM and TE components of the electric vector, and $\Delta$ is the difference between their phase lags at reflection.

In the particular case of a bulk material, expansion of equation (1.107) leads to the following expression of $n_{2}$ :

$$
n_{2}=n_{1} \sin \theta_{1} \sqrt{1+\left(\frac{1+\rho_{e}}{1-\rho_{e}}\right)^{2} \tan ^{2} \theta_{1}}
$$

\subsubsection{Two main methods}

Among the numerous methods used in ellipsometry, this section describes only some of the more common, namely the "nulling technique" and the "modulation technique by rotatable components". More complete reviews, concerning for example the techniques of "multiple angle of incidence ellipsometry (MAIE)", "spectroscopic ellipsometry (SE)", or "variable angle spectroscopic ellipsometry (VASE)", may be found in references [15-18, 27-29].....

\section{Nulling technique:}

The configuration of the nulling technique (figure 1.16) comprises a monochromatic source (laser or filtered source), a polarizer, a compensator (such as a quarter wave plate) an analyzer, and a detector. The linearly polarized output wave from the polarizer is converted into an elliptically polarized wave by the compensator, which is oriented in such a way that the light reflected by the sample is linearly polarized. The analyzer is then oriented perpendicularly to the plane of polarization in order to obtain extinction ("nulling") of the reflected beam. 


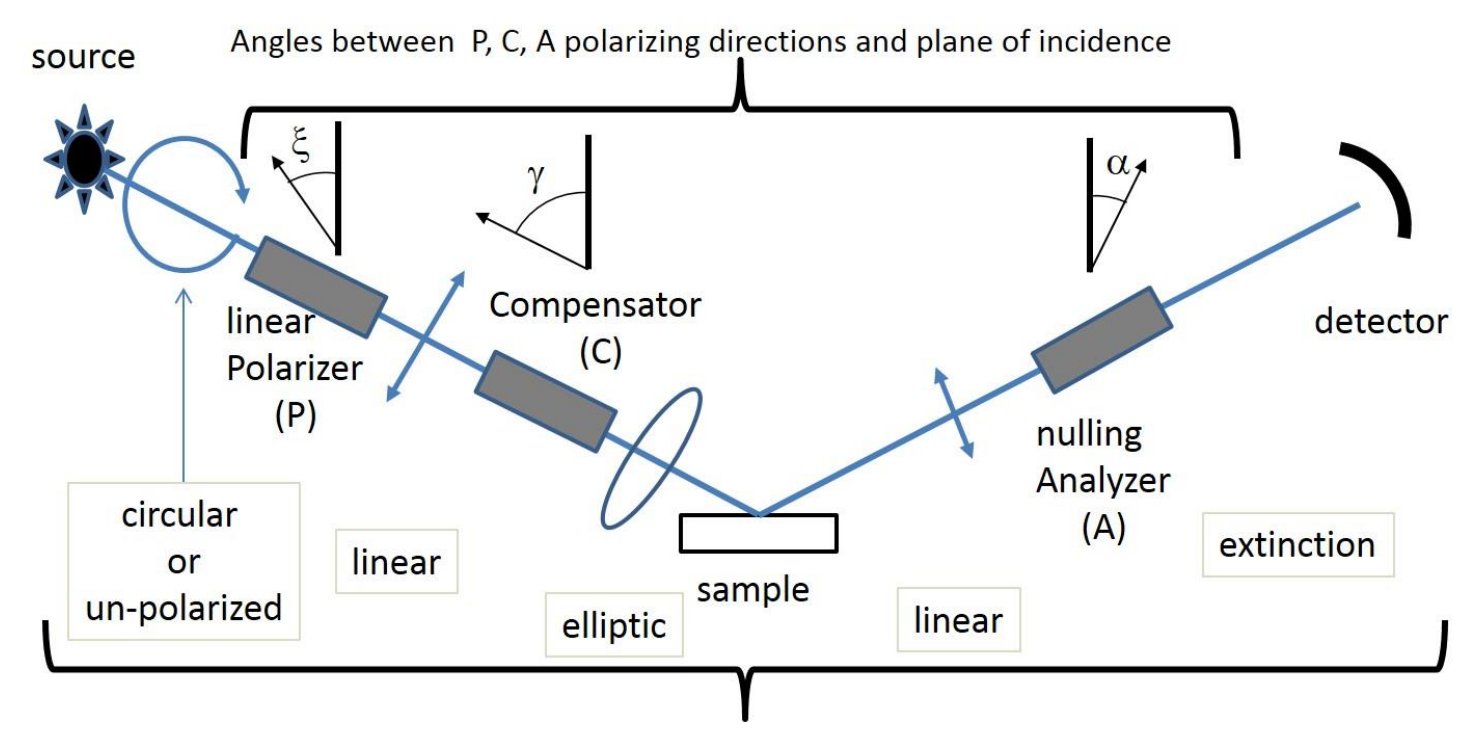

Figure 1.16: Configuration of a nulling ellipsometer

The ellipsometric parameters of the sample are directly derived from the respective orientation angles $\xi$ of the polarizer, $\gamma$ of the compensator and $\alpha$ of the analyzer, with respect to the plane of incidence, by means of the following relationship:

$$
\tan \psi \exp (i \Delta)=-\tan \alpha \frac{\tan \gamma-\tan (\xi-\gamma)}{1+i \tan \gamma \tan (\xi-\gamma)}
$$

Even when automatized, this method is rather slow because of the search for the minimum value of the output signal from the detector, but it is quite accurate

\section{- Modulation techniques by means of rotating components}

These techniques consist in periodically modulating the state of polarization of the beam by means of rotating either one of these components: the polarizer, the compensator, or the analyzer. If the rotating element is the polarizer, then the source must be circularly polarized, or un-polarized, as perfectly as possible. In case it is the analyzer, then the detector must not be polarization sensitive. If it is the compensator, these constraints on both the source and the detector are relaxed, but the compensator must be carefully calibrated spectrally and well aligned.

Time dependence of the detector output (case of an ellipsometer with rotating analyzer)

Let us consider the configuration of Figure 1.17 in which the (stationary) polarizer is linearly polarized along some axis at angle $\xi$ with the plane of incidence. 


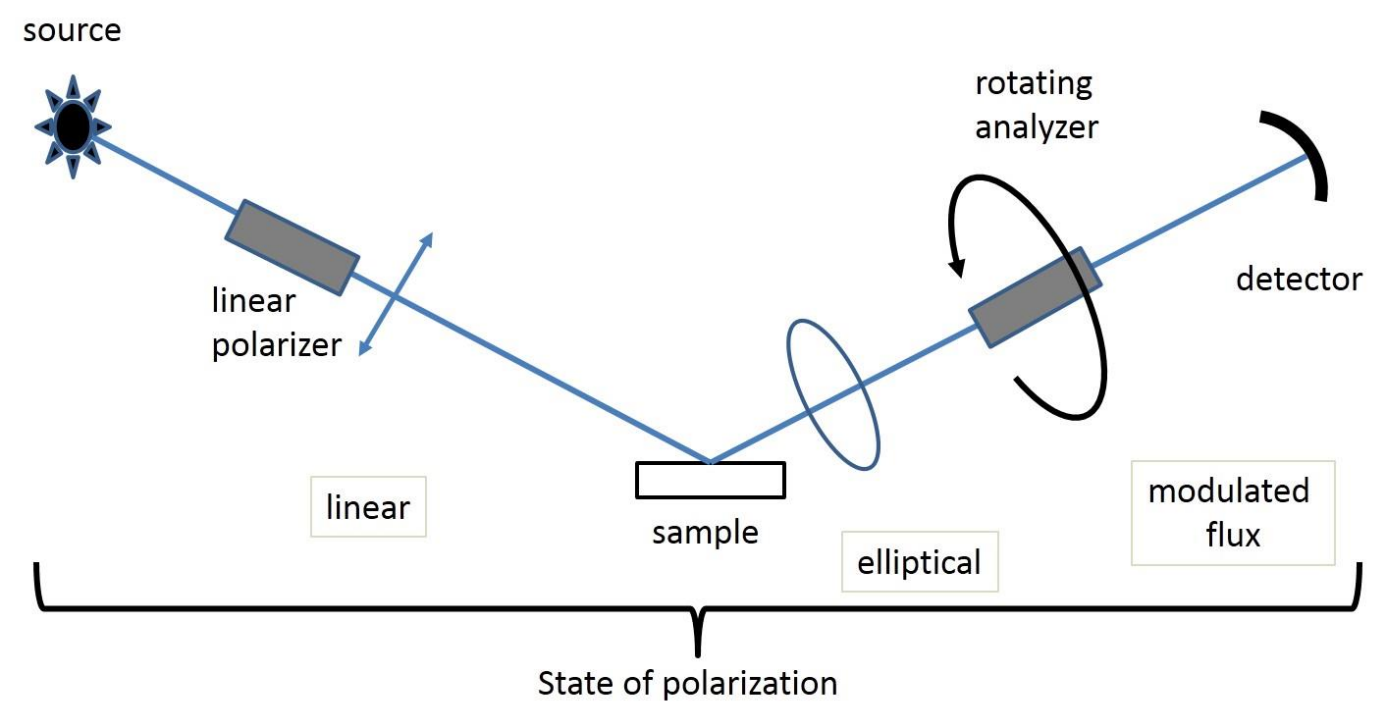

Figure 1.17: Modulation ellipsometer in the rotating analyzer configuration.

The parallel ( $p$ ) and perpendicular (s) components of the electric vector are then:

$$
E_{p}=E_{0} \cos \xi \text { and } E_{s}=E_{0} \sin \xi(1.110)
$$

After reflection of the wave from the sample, these components become:

$$
\begin{aligned}
& E_{p}^{\prime}=r_{/ /} E_{p}=r_{\perp} E_{0} \tan \psi \exp (i \Delta) \cos \xi \\
& E_{s}^{\prime}=r_{\perp} E_{s}=r_{\perp} E_{0} \sin \xi
\end{aligned}
$$

The complex amplitude a $(\mathrm{t})$ at the exit of the analyzer is obtained by summing the projections of the components $E_{p}^{\prime}$ and $E_{s}^{\prime}$ onto the polarizing direction of the analyzer (Figure 1.18), hence:

$$
a(t)=E_{p}^{\prime} \cos \Omega t+E_{s}^{\prime} \sin \Omega t(1.112)
$$

where $\Omega$ is the angular speed of rotation of the analyzer. 


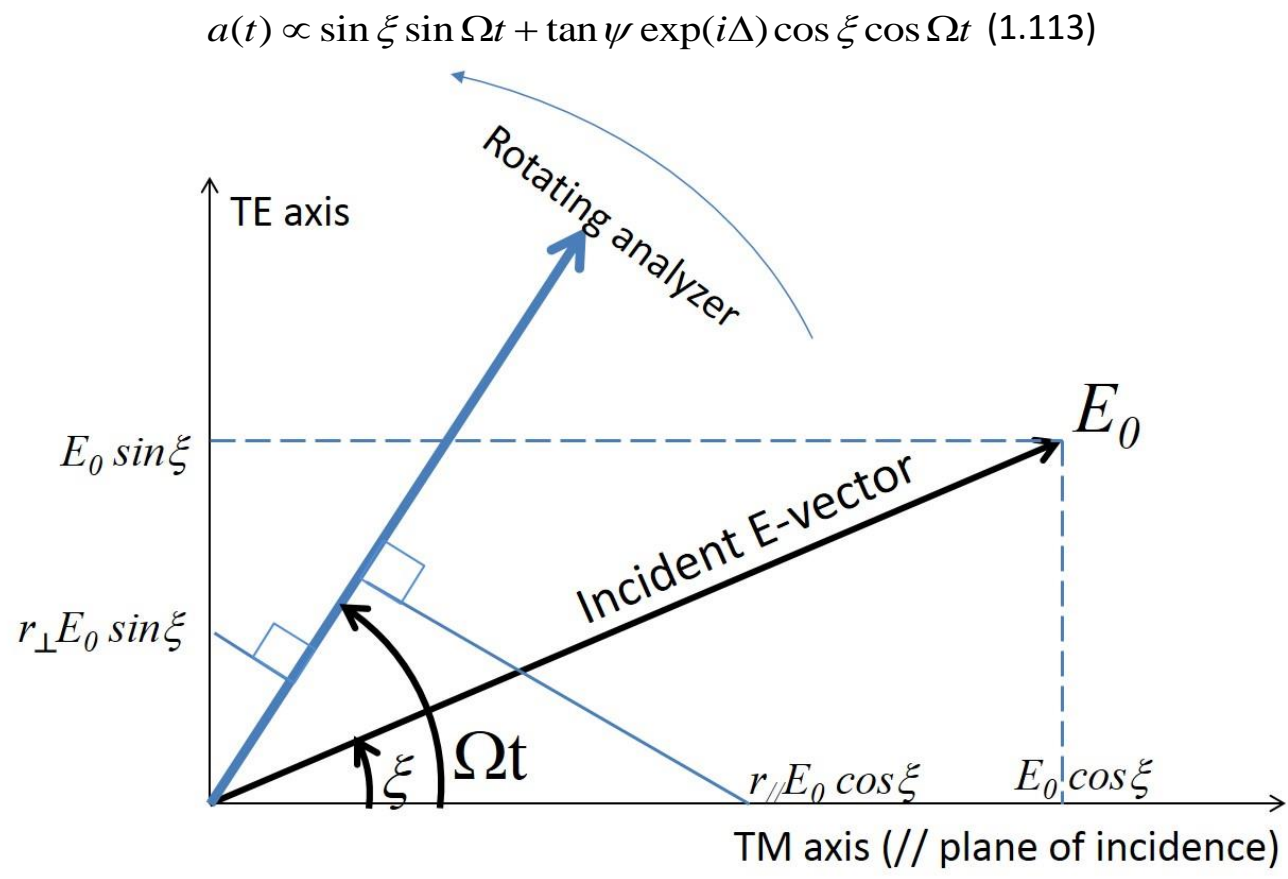

Figure1.18: States of polarization

Since the flux on the detector is proportional to the modulus square of the amplitude, one gets:

$$
F(t) \propto a(t) a^{*}(t)(1.114)
$$

$F(t) \propto[\sin \xi \sin \Omega t+\tan \psi \exp (i \Delta) \cos \xi \cos \Omega t][\sin \xi \sin \Omega t+\tan \psi \exp (-i \Delta) \cos \xi \cos \Omega t]$ (1.115)

After development and simplification, the following relationship is obtained:

$F(t) \propto \tan ^{2} \psi \cos ^{2} \xi+\sin ^{2} \xi+\cos 2 \Omega t\left[\tan ^{2} \psi \cos ^{2} \xi-\sin ^{2} \xi\right]+\sin 2 \Omega t \sin 2 \xi \tan \psi \cos \Delta$ (1.116)

Hence the signal flux upon the detector is a periodic function of time, which varies at twice the frequency of rotation of the analyzer. So does the output current $I(t)$ from the detector, which can be expressed as follows with respect to its average value $I_{0}$ :

$$
\frac{I(t)}{I_{0}}=1+\mathscr{Q} \cos 2 \Omega t+\mathscr{B} \sin 2 \Omega t(1.117)
$$

Where $\mathscr{A}$ and $\mathcal{B}$ are the Fourier coefficients of this function at its fundamental frequency, with:

$$
\mathscr{A}=\frac{\tan ^{2} \psi \cos ^{2} \xi-\sin ^{2} \xi}{\tan ^{2} \psi \cos ^{2} \xi+\sin ^{2} \xi}=\frac{-1+\tan ^{2} \psi \cot ^{2} \xi}{1+\tan ^{2} \psi \cot ^{2} \xi}
$$

and 


$$
\mathscr{B}=\frac{\sin 2 \xi \tan \psi \cos \Delta}{\tan ^{2} \psi \cos ^{2} \xi+\sin ^{2} \xi}=\frac{2 \tan \xi \tan \psi \cos \Delta}{1+\tan ^{2} \psi \cot ^{2} \xi}
$$

The ellipsometric angles $\psi$ and $\Delta$ of the sample are then derived from the above quantities by:

$$
\tan \psi=\left|\sqrt{\frac{1+\mathscr{Q}}{1-\mathscr{Q}}}\right| \tan \xi \mid \text { and } \cos \Delta=\mathscr{B}\left|\sqrt{\frac{1}{1-\mathscr{Q}^{2}}}\right|
$$

The sign of $\Delta$, which depends upon the (left or right hand) type of the elliptical polarization of the reflected beam, cannot be determined from the flux detected in this set-up. In order to solve this ambiguity, a complementary measurement is needed, by means of a compensator of known slow and fast axes.

\subsection{Temperature dependence of the refractive index}

\subsubsection{Basic considerations}

The temperature is one of the most important parameter affecting the value of the refractive index of bulk (dielectric) optical materials. At a macroscopic scale, we consider the material as a stack of identical molecules each one being linked to each other through some kind of binding forces. From Lorentz's theory mentioned in subsection 1.1.2.2, if $p$ is the elemental polarization associated to a

molecule placed in an electric field $\boldsymbol{E}$, we have $\boldsymbol{p}=\alpha_{p} \varepsilon_{0} \boldsymbol{E}$ where $\alpha_{p} \quad$ represents the molecular polarizability. The polarization $\boldsymbol{P}$ at the scale of the unit volume $V$ of material will be $\boldsymbol{P}=N \boldsymbol{p}=N \alpha_{p} \varepsilon_{0} \boldsymbol{E}, N$ being the density number of molecules. For one mole of matter for instance, we have $\boldsymbol{P}=\frac{N_{A} \rho_{m} \alpha_{p}}{M} \varepsilon_{0} \boldsymbol{E}, N_{A}$ being the Avogadro number, $M$ the molecular weight and $\rho_{m}$ the mass density. In that case the volume $V$ that is to be considered will be the molar volume. Both $\rho_{m}$ and $\alpha_{p}$ parameters are temperature dependent and, consequently, so it is for the refractive index.

Several attempts have been made to describe as accurately as possible the thermo-optic coefficient (TOC) $\frac{d n}{d T}$ of optical materials [30-36]. Predicting their thermal behavior over the whole spectral range of transparency is also of most importance for proper implementation in non linear optical devices where high power lasers are employed in frequency conversion arrangements.

One way to evaluate the TOC may be to start from the Clausius-Mossotti relationship (analogous to equation (1.35) which connects the dielectric permittivity $\varepsilon$ of a medium to its microscopic polarizability $\alpha_{p}$. It was first proposed for static electric fields and then extended to the case of 
alternating ones in the framework of the local field theory developed by $\mathrm{H}$. Lorentz. For one mole of matter, the formula is written as:

$$
\frac{\varepsilon-1}{\varepsilon+2}=\frac{N_{A} \rho_{m} \alpha_{p}}{3 M}
$$

Taking the derivative with respect to $T$ gives:

$$
\frac{3}{(\varepsilon+2)^{2}} \frac{\partial \varepsilon}{\partial T}=\frac{N_{A}}{3 M}\left\{\alpha_{p} \frac{\partial \rho_{m}}{\partial T}+\rho_{m}\left[\left(\frac{\partial \alpha_{p}}{\partial T}\right)_{T} \frac{d V}{d T}+\left(\frac{\partial \alpha_{p}}{\partial T}\right)_{V}\right]\right\}
$$

for which a dependence of $\alpha_{p}$ on both $V$ and $T$ is assumed. For an isotropic material and considering a volume $V$ as the one of a sphere of radius $R$, we have $\frac{d V}{d T}=3 V \alpha_{L}, \alpha_{L}$ being the usual linear thermal expansion coefficient: $\alpha_{L}=\frac{1}{R} \frac{d R}{d T}$. Recasting equation (1.122) then yields:

$$
\frac{1}{(\varepsilon-1)(\varepsilon+2)} \frac{\partial \varepsilon}{\partial T}=-\alpha_{L}\left[1-\frac{V}{\alpha_{p}}\left(\frac{\partial \alpha_{p}}{\partial V}\right)_{T}\right]+\frac{1}{3 \alpha_{p}}\left(\frac{\partial \alpha_{p}}{\partial T}\right)_{V}
$$

For materials exhibiting a high thermal expansion coefficient, like alkali halides for instance, the TOC is negative, while being positive in covalent compounds due to a pre-eminent contribution of the change in polarizability. It can be predicted also from equation (1.135) described further in subsection 1.3.3.1 that the thermo-optic coefficient in the transparent region is decreasing with angular frequency $\omega$ while becoming divergent in the vicinity of both UV and IR cutting edges.

Ramachandran [36] took also into account the change of density, which induces a net change in polarizability, added to a change in polarizability due only to the change in temperature. This is formally equivalent to what can be identified in formula (1.122). From this point of view, he derived the following equation describing the TOC of optical glasses:

$$
\frac{d n}{d T}=\frac{n^{2}-1}{2 n}\left(-\alpha_{\mathrm{v}}+\Lambda_{0} \alpha_{\mathrm{v}}+\tau_{0}\right)(1.124)
$$

after having shown that the polarizability $\alpha_{p}$ may exhibit a linear dependence on temperature $T$ and written as:

$$
\alpha_{p}(T)=\alpha_{p_{0}}\left[1+\left(\Lambda_{0} \alpha_{\mathrm{v}}+\tau_{0}\right) T\right]
$$

$a_{v}$ is the volume expansion coefficient, i. e. $\frac{1}{V} \frac{d V}{d T}$; the parameters $\Lambda_{0}$ and $\tau_{0}$ have been recast in the form:

$$
\Lambda_{0}=1-\frac{2 n}{n^{2}-1} \frac{\rho_{m} \partial n}{\partial \rho_{m}}
$$




$$
\tau_{0}=\frac{d\left[\ln \left(n^{2}-1\right)\right]}{d T}+\alpha_{\mathrm{v}} \frac{2 n}{n^{2}-1} \frac{\rho_{m} \partial n}{\partial \rho_{m}}
$$

Thermo-optic measurements were performed on several glasses and cubic ionic crystals. It appeared that for glasses, the change in polarizability due to pure temperature effect is dominant as compared to the contribution of volume variation. The converse was true for ionic crystals where the change in the lattice parameter becomes dominant, leading to a negative value of $d n / d T$. This behavior is illustrated in table 1.4 which gives for comparison $d n / d T$ and $\alpha_{\mathrm{v}}$ data known for ionic cubic crystals (alkali halides la - VIla and alkaline earth fluorides $\mathrm{CaF}_{2}, \mathrm{BaF}_{2}$ ) and for some typical Schott, Hoya, Hikari, Ohara and specialty IR glasses.

\begin{tabular}{|c|c|c|c|c|}
\hline Compound & Wavelength $(\mu \mathrm{m})$ & $d n / d \operatorname{Tx} 10^{6}\left(\mathrm{~K}^{-1}\right)$ & $\alpha_{\mathrm{v}} \times 10^{6}\left(\mathrm{~K}^{-1}\right)^{(1)}$ & References \\
\hline \multicolumn{5}{|c|}{ Ionic crystals } \\
\hline LiF & 0.633 & $-18^{\mathrm{a}, \mathrm{b}}$ & $\begin{array}{c}99.6^{\mathrm{a}} \\
102.9^{\mathrm{b}}\end{array}$ & $\begin{array}{c}\text { [42], [40], [44] } \\
{[38],[37]^{\mathrm{b}}}\end{array}$ \\
\hline $\mathrm{LiCl}$ & 0.633 & $-31.7^{a}$ & $131.4^{\mathrm{a}, \mathrm{b}}$ & {$[38],[37],[42]$} \\
\hline $\mathrm{LiBr}$ & 0.633 & $-38.8^{a}$ & $150^{a, b}$ & [38], [37], [43] \\
\hline Lil & 0.633 & $-48^{a}$ & $177^{\mathrm{a}, \mathrm{b}}$ & {$[38]$} \\
\hline $\mathrm{NaF}$ & 0.633 & $-16.8^{a}$ & $100.5^{a, b}$ & {$[38],[37],[42],[44]$} \\
\hline $\mathrm{NaCl}$ & 0.633 & $-32.2^{a}$ & $123.3^{a, b}$ & {$[38],[42],[44]$} \\
\hline $\mathrm{NaBr}$ & 0.633 & $-38.6^{a}$ & $125.4^{a, b}$ & {$[38],[42],[44]$} \\
\hline $\mathrm{Nal}$ & 0.633 & $-45.9^{a}$ & $134.1^{\mathrm{a}, \mathrm{b}}$ & [38], [42], [44] \\
\hline $\mathrm{KF}$ & 0.633 & $-23.2^{a}$ & $94.2^{a, b}$ & [38], [42], \\
\hline $\mathrm{KCl}$ & 0.633 & $-31.7^{a}$ & $109.5^{a, b}$ & [38], [42], \\
\hline $\mathrm{KBr}$ & 0.633 & $-36.6^{a}$ & $115.5^{a, b}$ & [38], [42] \\
\hline $\mathrm{KI}$ & 0.633 & $-43.75^{a}$ & $120.9^{a, b}$ & [38], [42] \\
\hline $\mathrm{RbF}$ & 0.633 & $-25.1^{\mathrm{a}}$ & $82.5^{a, b}$ & [42] \\
\hline $\mathrm{RbCl}$ & 0.633 & $-39.4^{a}$ & $108^{a, b}$ & [38] \\
\hline $\mathrm{RbBr}$ & 0.633 & $-44.9^{a}$ & $111^{a, b}$ & [38] \\
\hline $\mathrm{Rbl}$ & 0.633 & $-56.3^{a}$ & $117^{\mathrm{a}, \mathrm{b}}$ & [38], [37] \\
\hline CsF & 0.633 & $-41.7^{a}$ & $111^{a, b}$ & [40] \\
\hline $\mathrm{CsCl}$ & 0.633 & $-77.4^{a}$ & 135 & [38], [37] \\
\hline $\mathrm{CsBr}$ & 0.633 & $-84.75^{a}$ & $141.6^{\mathrm{a}, \mathrm{b}}$ & [37], [44] \\
\hline CsI & 0.633 & $-96.25^{a}$ & $145.8^{a, b}$ & [37], [44] \\
\hline $\begin{array}{c}\text { KRS } 5 \\
\text { (TIBr 48\%, TII 52\%) }\end{array}$ & 10.6 & -235 & 174 & [44] \\
\hline $\mathrm{CaF}_{2}$ & 0.633 & -10.4 & 56.7 & $\begin{array}{c}\text { [41], [43], [51], [52], } \\
{[44]}\end{array}$ \\
\hline $\mathrm{SrF}_{2}$ & 0.633 & -12.7 & 55.2 & {$[41],[44]$} \\
\hline $\mathrm{BaF}_{2}$ & 0.633 & -16 & 55.2 & {$[41],[52],[44]$} \\
\hline \multicolumn{5}{|c|}{ U. V. - Vis. Selected Glasses } \\
\hline (2) Lithosil $\mathrm{SiO}_{2}$ & 0.633 & 10 & 1.56 & [43] \\
\hline${ }^{(2)} \mathrm{N}-\mathrm{BK} 7$ & 0.633 & 2.5 & 21.9 & {$[46],[48],[49]$} \\
\hline${ }^{(2)} \mathrm{N}-\mathrm{SF} 6$ & 0.633 & 0.43 & 27 & {$[46],[48],[49]$} \\
\hline (2) LF5 & 0.633 & 2.3 & 24.9 & {$[46],[46],[49]$} \\
\hline
\end{tabular}




\begin{tabular}{|c|c|c|c|c|}
\hline $\begin{array}{c}\text { Clearceram-Z } \\
\text { regular }\end{array}$ & 0.633 & 13.9 & 6 & [45] \\
\hline \multicolumn{5}{|c|}{ Specialty IR Glasses } \\
\hline $\begin{array}{c}\text { Germanate glass } \\
9754\end{array}$ & 1.060 & 10.4 & 18.6 & [43] \\
\hline Amtir $6\left(\mathrm{As}_{40} \mathrm{~S}_{60}\right)$ & 5 & $\begin{array}{c}-8.6(25 \text { to }- \\
\left.78^{\circ} \mathrm{C}\right) ;+9.3(20 \\
\left.\text { to } 65^{\circ} \mathrm{C}\right)\end{array}$ & 64.2 & [51] \\
\hline $\begin{array}{l}\text { IRG } 6 \text { Schott } \\
\left(\mathrm{As}_{40} \mathrm{Se}_{60}\right)\end{array}$ & 5 & 33.5 & 62.4 & [46] \\
\hline Gasir 1 & 10.6 & 55 & 51 & [50] \\
\hline Gasir 5 & 10.6 & 32 & 23.5 & [50] \\
\hline IRG 22 & 5 & 67.7 & 36.3 & [46] \\
\hline $\begin{array}{c}\text { IG } 2 \\
\left(\mathrm{Ge}_{33} \mathrm{As}_{12} \mathrm{Se}_{55}\right)\end{array}$ & 3.39 & 67.7 & 36.3 & [47] \\
\hline IRG 23 & & 103.8 & 40.2 & [46] \\
\hline $\begin{array}{c}\text { IG } 3 \\
\left(\mathrm{Ge}_{30} \mathrm{As}_{13} \mathrm{Se}_{32} \mathrm{Te}_{25}\right) \\
\end{array}$ & 3.39 & 105.2 & 40.2 & [47] \\
\hline IRG 24 & 5 & 21.5 & 61.2 & [46] \\
\hline $\begin{array}{c}\text { IG } 4 \\
\left(\mathrm{Ge}_{10} \mathrm{As}_{40} \mathrm{Se}_{50}\right)\end{array}$ & 3.39 & 23 & 61.2 & [47] \\
\hline $\begin{array}{c}\mathrm{AMTIR} 1 \\
\left(\mathrm{Ge}_{33} \mathrm{As}_{12} \mathrm{Se}_{55}\right)\end{array}$ & 3.39 & 77 & 36 & [51] \\
\hline AMTIR 2 (AsSe) & 10.6 & 30 & 67.2 & [51] \\
\hline ZBLAN & 0.633 & -14.75 & 60 & {$[52]$} \\
\hline
\end{tabular}

Table 1.4: Thermo-optic coefficient $d n / d T\left(x 10^{6} K^{-1}\right)$ and volume expansion coefficient $\alpha_{v}\left(x 10^{6} K^{-1}\right)$ of some ionic crystals and selected glasses.

${ }^{(1)}$ Values deduced from referenced linear $\alpha_{\mathrm{L}}$ expansion coefficients: $\alpha_{\mathrm{v}}=3 \alpha_{\mathrm{L}}$

(2) Selected glasses are referred to Schott catalog denomination [46]; for the same glasses different names can be found in ref [45], [48] and [49]. A convenient comparative table is given in ref. [45].

${ }^{\text {a }}$ values calculated @ 0.6328 (He-Ne laser line) from recommended data of ref [40]

${ }^{b}$ from ref [37], [39]

\subsubsection{Measurement of the temperature dependence of the refractive index $n(\lambda, T)$}

\subsubsection{Direct measurement}


The temperature dependence of the refractive index $n(\lambda, T)$ can be obtained directly by using the minimum deviation method if the prism is mounted in an adequate temperature controlled cell and illuminated with an appropriate wavelength selecting set-up. Indeed, among the various methods described in section 1.2, it appears as the most suitable one to achieve best accuracy. However, if a wide temperature range is to be explored, this would require the use of an air-tight evacuated and thermal insulated enclosure, along with additional optical components allowing entering and leaving of the light beam, which obviously will alter the accuracy of measurements. Such problems have been overcome to some extent at NASA Goddard Space Flight Center with the development of the Cryogenic, High-Accuracy Refraction Measuring System (CHARMS) equipment which enables accurate measurements of $n(\lambda, T)$, mostly dedicated to proper implementation of optical materials for space applications at cryogenic temperatures $[10,11,53,54]$. Even appearing as well suited for isotropic, homogeneous and perfectly transparent materials, some disadvantages can still be mentioned:

- It is known that best accuracy is obtained with prisms of quite large dimension (say a few $\mathrm{cm}^{2}$ in aperture), leading to considerable thermal time constants and to an eventual occurrence of thermal gradients in the sample. The measurement procedure is of step by step type, with regularly spaced temperature intervals and suitable soaking times of the sample. These operating constraints induce unavoidable rather consuming time of measurements.

-Submitted to a rise in temperature, any (homogeneous) material would exhibit an increase in its absorption coefficient and, consequently, the beam could progressively lose more or less its original transverse symmetry of intensity while travelling inside the prism. This is particularly true for semiconductors like (cubic) germanium for instance. For large temperature excursion and/or rather high values of absorption such phenomenon could lead to a shift of the maximum of irradiance onto the pupil of the detection system and a slight widening of the diffraction pattern in the focal plane of the detection system. This qualitative description is illustrated figure 1.19 which shows that the location of the irradiance maximum is shifted towards the summit of the prism.

- Case of anisotropic materials: accurate measurements are to be performed in polarized light and require a set of prims which are to be precisely cut with respect to appropriate crystallographic directions which depend on the space group of the compound. This can be adequately realized at room temperature, say $T_{0}$, and the principal refractive indices could thus accurately be measured at $T_{0}$ using the minimum of deviation method. However, if the compound undergoes a temperature change from $T_{0}$ to $T$, its intrinsic anisotropic character will induce internal strains; these ones could distort the initial shape of the prism, depending on the symmetry class that it belongs to [55]. Thus, dealing with thermal behaviour of refractive indices, the minimum of deviation method appears not very convenient for this purpose.

Most of the above mentioned difficulties inherent to direct measurements of the refractive index as function of temperature can be overcome by using differential interferometric methods which are described in the next section 


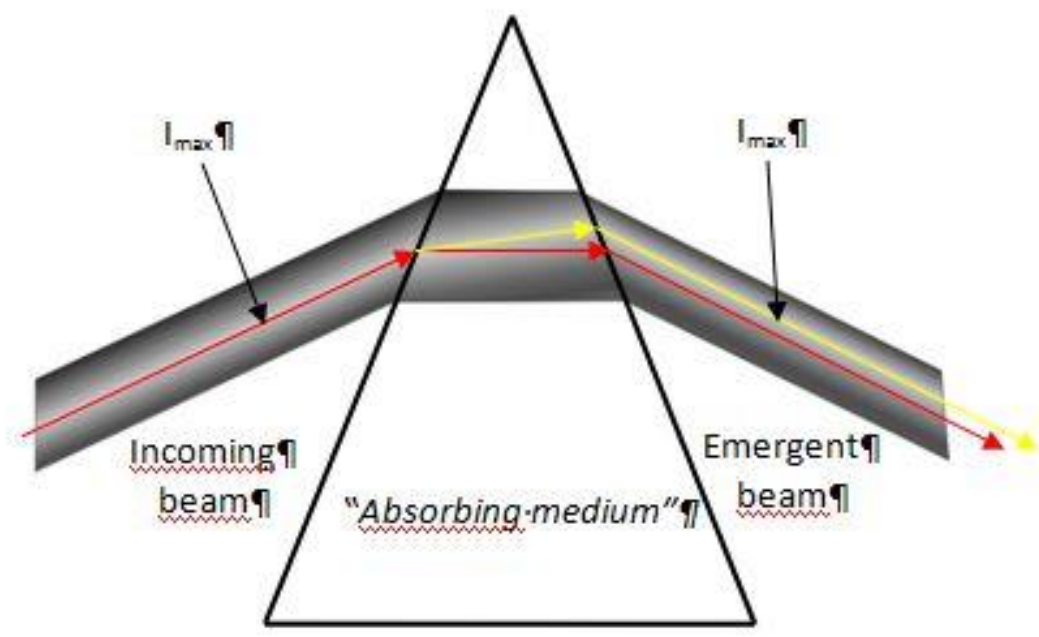

Figure 1.19: Prism method in the case of an "absorbing material". The transverse profile of intensity becomes asymmetric at the exit of the prism.

\subsubsection{Interferometric methods: normalized thermo-optic coefficients (NTOC)}

It is known that interferometric methods may be used advantageously to determine the thermo-optic coefficients with high accuracy. However they give only what we have called the "normalized thermooptic coefficient" [56, 57] (NTOC), namely

$$
\beta_{N T O C}=\frac{1}{n} \frac{d n}{d T}(1.128)
$$

instead of the usual and commonly admitted one TOC, defined as $d n / d T$. Temperature dependence measurements of NTOC's can be performed at several discrete laser wavelengths by using specifically developed interferometric arrangements that allow accuracy around a few in $10^{-6} \mathrm{~K}^{-1}$ ) [58]. Also data can be obtained on relatively small parallelepipedic shaped specimen (say a few $\mathrm{mm}^{2}$ in aperture and about $7 \mathrm{~mm}$ in length), that ensures for low thermal time constant while overcoming the problem of non-homogeneous absorption described above.

The first attempts to get accurate measurements using interferometric means could be attributed to set-ups developed at the U.S. National Bureau of Standards [59], improved for years and used to characterize the thermal behavior of numerous materials [60-62]. Of course, many techniques were then elaborated, employing various Fizeau, Fabry-Pérot, Michelson or Mach-Zehnder based interferometric arrangements. Some examples will be found in references [63-65]. In all cases, the principle is based on measuring the number of fringes $N$ passing in front of a detector when submitting 
a sample to a temperature change $\Delta T$. Regardless of the technique employed, the determination of the temperature dependence of the refractive index requires measurements of both thermal expansion and changes in optical path. For a sample of thickness $L$ and refractive index $n$, the linear thermal expansion and normalized thermo-optic coefficient (NTOC) are given by $\left.\alpha_{L}=d[\ln (L)]\right) / d T$ and $\beta_{\text {NTOC }}=d[\ln (n)] / d T$, respectively. Defining as well the coefficient $\gamma$ for "normalized thermal changes in optical path" (NTCOP), that is $\gamma \mathrm{NTCO}=\mathrm{d}[\ln (L n)] / \mathrm{dT}$, the NTOC is obtained from the straightforward relationship $\beta_{N T O C}=\gamma_{N T C O P}-\alpha_{L}$. As an example we describe hereafter an original arrangement that was specifically designed for the determination of the thermo-optic coefficients of bulk nonlinear optical (NLO) materials [58], for which an accuracy on $d n / d T$ of about $10^{-5}$ is required. Obviously, its use can easily be extended to any optical one, and particularly to (isotropic) glasses, seeing that in such case and for NTCOP measurements the polarization state of the beam with respect to dielectric axes of the medium is not so stringent.

\subsection{Dilatometric measurements}

Measurements of linear thermal expansion are performed by using an absolute laser interferometric dilatometer acting as an optical gauge. The corresponding modified Mach-Zehnder set-up is entirely mounted in a vacuum enclosure and provided with a thermostated base plate; it is depicted in Figure 1.20. The parallelepipedic-shaped sample $\mathbf{S}$ is located at the center of an oven in a nearly blackbody configuration to avoid any occurrence of thermal gradient. Two silica windows $W_{1}$ and $W_{2}$ allow entering and leaving of light from the vacuum enclosure. The beam of a frequency stabilized He-Ne laser is divided into two paths by the beam splitter $\mathrm{BS}_{1}$. The plane and parallel optical end faces of the specimen are gold metallized and act as mirrors $M_{5}$ and $M_{6}$ in the sample arm. After reflection on the front face $M_{5}$, the beam is sent by successive reflections on $B S_{1}, M_{2}, M_{4}$, and beam splitter $B S_{2}$ to the back surface $M_{6}$ of $\boldsymbol{S}$. The reflected beam overlaps then the one of the reference arm (path $B S_{1}, M_{1}, M_{3}$ and RP) at the recombination plate RP to give a fringe pattern which is recorded by photo-detector $\mathrm{D}_{1}$. Detection $D_{2}$, external to the enclosure, enables also convenient observation of the fringe pattern on a screen and verification of its spatial stability. Best accuracy of measurements is achieved by inserting a piezo-transducer PZT on mirror $\mathrm{M}_{1}$ that allows phase modulation and subsequent phase detection of the fringe shift induced by applying a linear ramp of temperature to the sample through temperature control TC (Pt 100 thermistor) of the oven. The temperature of the sample is given by a $\mathrm{Cu} /$ constantan thermocouple glued on one of its sides. The optical source is a frequency/intensity stabilized He-Ne laser emitting at wavelength $\lambda$; a phase shift of $2 \pi$ (i. e. fringe spacing), induced by a variation of temperature $\Delta T$, corresponds to a change in length $L$ of $\lambda / 2$. With phase modulation, two consecutive zero responses on the photodetector $D_{1}$ correspond to an optical path variation of $\lambda / 4$. Thus, if $N$ is the number of recorded fringes in the first case, the linear expansion coefficient will be given by $\alpha_{L}=\frac{N \lambda}{2 L \Delta T}$. In the second case we will get twice more fringes, and which are moreover detected unambiguously while crossing a zero response on $D_{1}$. With such improvement, the typical accuracy on linear dilatation coefficient measurements is close to $3 \times 10^{-7} \mathrm{~K}^{-1}$ for a sample of $5 \mathrm{~mm}$ in length examined over a $10 \mathrm{~K}$ temperature interval. It is temperature dependent, going down to zero at $T=0 \mathrm{~K}$. As long as the material does not exhibit strong structural modification within the explored temperature interval, $\alpha_{L}(T)$ may always be fitted accurately to a power series in $T$ as: 


$$
\alpha_{L}(T)=a_{0}+\sum_{k=1}^{m} a_{k} T^{k}
$$

A schematic lay-out and a picture of the apparatus are given figure 1.20 and 1.21 , respectively.

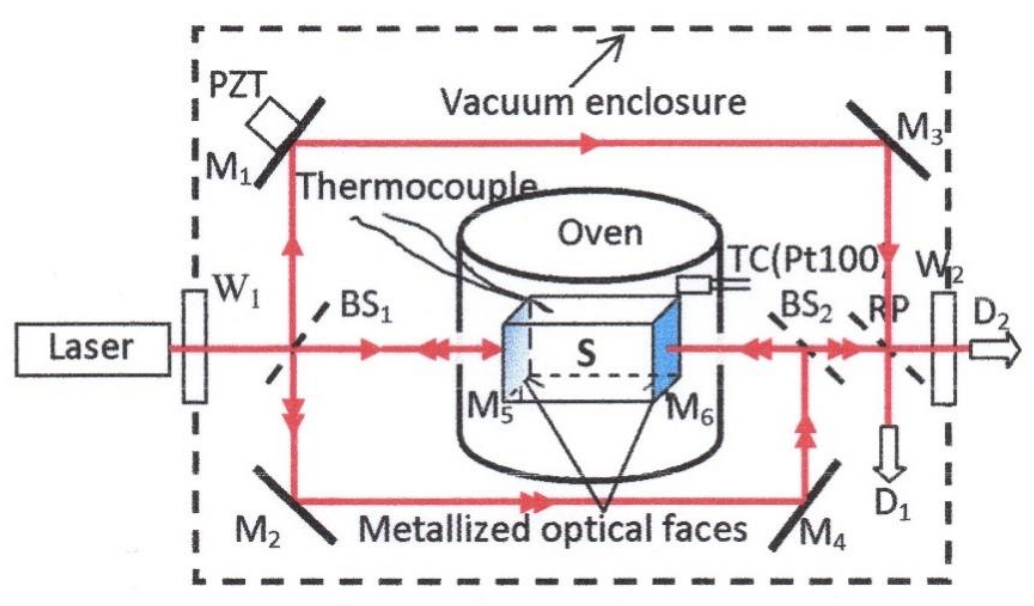

Figure 1.20: Lay-out of an absolute interferometric dilatometer used for thermal expansion measurements.

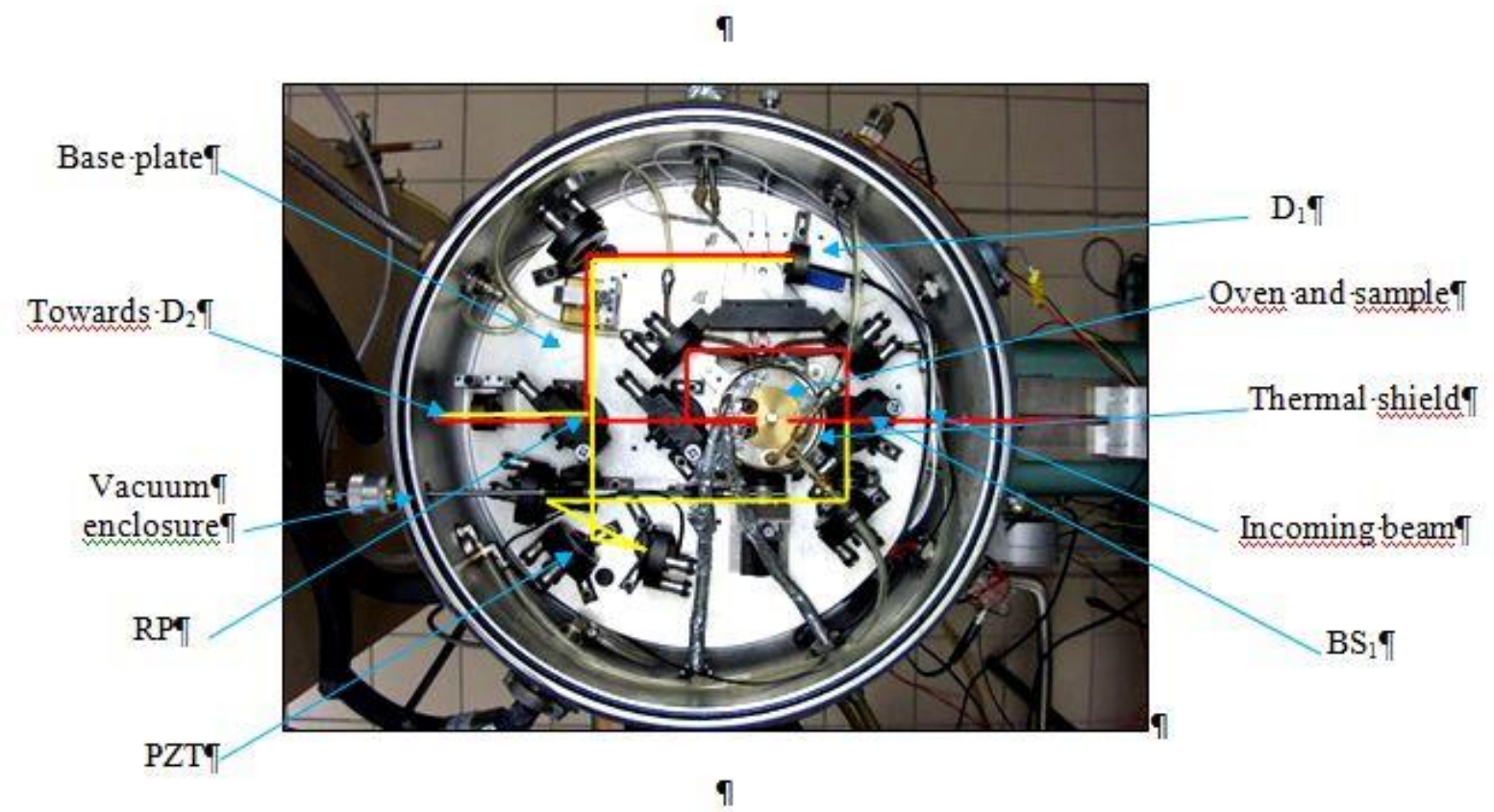

Figure 1.21: Photograph of an absolute interferometric dilatometer; red and yellow colored beams correspond to the sample and reference arms, respectively. Not shown: the laser source located at the 


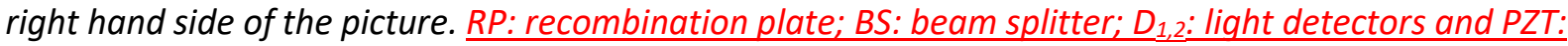
piezotransducer.

\subsection{Determination of normalized thermo-optic coefficients}

Normalized thermal changes in optical path NTCOP $\gamma_{\text {NTCOP }}$ are obtained by removing metallization $\mathrm{M}_{5}$ and $M_{6}$. The same sample $S$ is mounted in a specific vacuum cell which reproduces identical thermal working conditions as those used in the dilatometer. Appropriate translation and rotation stages enable accurate orientation of the sample with respect to the direction of the incident beam. A FabryPérot interference pattern is generated by multiple reflections on the optical end faces; the fringe shift induced by applying a linear ramp of temperature to the sample is observed by reflection and continuously recorded on photo-detector $D_{1}$ after being reflected by the semi reflecting plate $B S$, as shown in figure 1.22. An analyzer crossed with respect to the orientation of the front polarizer allows permanent control of the spatial stability and eventual structural changes of the sample all along heating runs by recording on detector $\mathrm{D}_{2}$ the intensity of the transmitted beam which is imaged outside the vacuum cell. $\mathrm{BaF}_{2}$ windows $\mathrm{W}_{1}$ and $\mathrm{W}_{2}$ enable convenient switching of laser sources from the UV$V$ is up to the IR spectral ranges. With such arrangement, the typical accuracy in the determination of NTCOP of a sample similar to the one described above and exhibiting a refractive index $n=1.5$ with an uncertainty around $10^{-4}$, is close to $2 \times 10^{-7} \mathrm{~K}^{-1}$. Accuracy in the NTOC determination $\beta_{T O C}=\gamma_{N T C O P}-\alpha_{L}$ may thus be expected to lay around less than a few in $10^{-6} \mathrm{~K}^{-1}$.

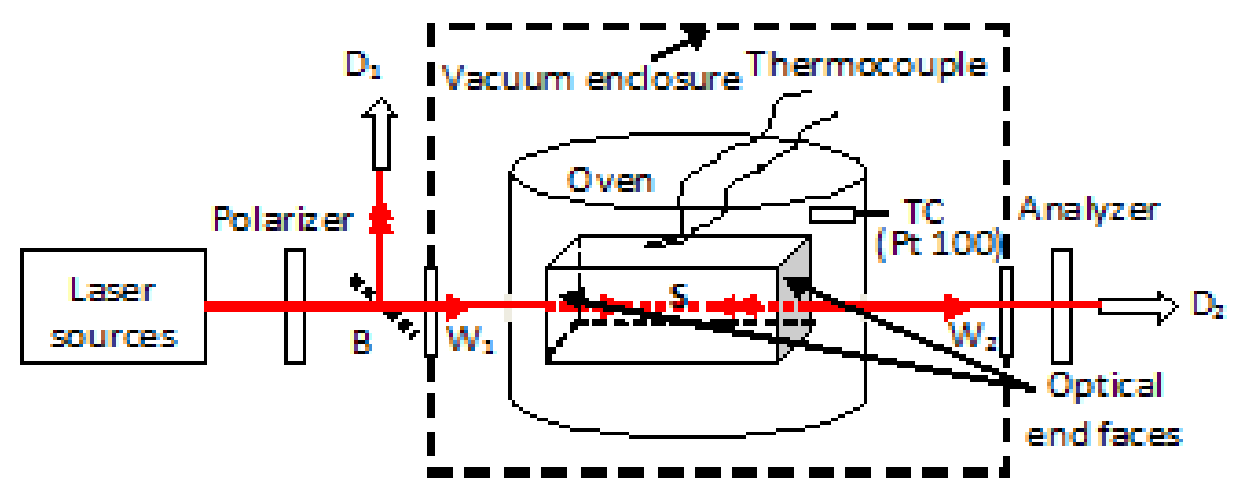

Figure 1.22: Optical lay-out used for measurements of thermal changes in optical path.

\subsubsection{Thermo-optic and refractive index dispersion}

\subsubsection{Theoretical considerations}


In a way somewhat similar to the works proceeding from a microscopic description of the matter, and which have lead for instance to the Lorentz-Lorenz and Sellmeier formula, G. Gosh [66] proposed a model based on a 3 energy levels of absorption between the valence band and the conduction band of the material. The characteristic energies associated to each process are $E_{\text {elec }}, E_{\text {isen }}$, and $E_{\text {excit }}$, corresponding to so-called average electronic, isentropic and excitonic absorption band gaps, respectively. $E_{\text {elec }}$ is the gap energy between the valence and the conduction bands, $E_{\text {excit }}$ corresponds to the energy of creation of an electron-hole entity while $E_{\text {isen }}$ is defined as a fitting gap that lies between the excitonic band and the conduction one. The energy levels are depicted in figure $1.23 \mathrm{In}$ the case of isotropic media, considering that the isentropic band is the geometric mean of the electronic absorption band and the excitonic one, further analysis gave the following temperature dependence of the refractive index $n$ linked to its thermo-optic coefficient $d n / d T$ :

$$
\begin{aligned}
& \qquad 2 n \frac{d n}{d T}=\left(n_{0}^{2}-1\right)\left(-3 \alpha_{L} \Gamma-\frac{1}{E_{\text {excit }}} \frac{d E_{\text {excit }}}{d T} \Gamma^{2}\right) \text { (1.130) } \\
& \alpha_{L} \text { is the linear thermal expansion coefficient, } \Gamma=\frac{\lambda^{2}}{\lambda^{2}-\lambda_{i}^{2}}
\end{aligned}
$$

$\Gamma$ is called "the normal dispersive parameter", $\lambda$ being the photon wavelength and $\lambda_{i}$ the isentropic band wavelength, lying in the UV region between the excitonic and the electronic absorption bands.

$n_{0}$ is the low-frequency refractive index in the IR region. The shift of lattice absorption with temperature is assumed to be negligibly small [66].

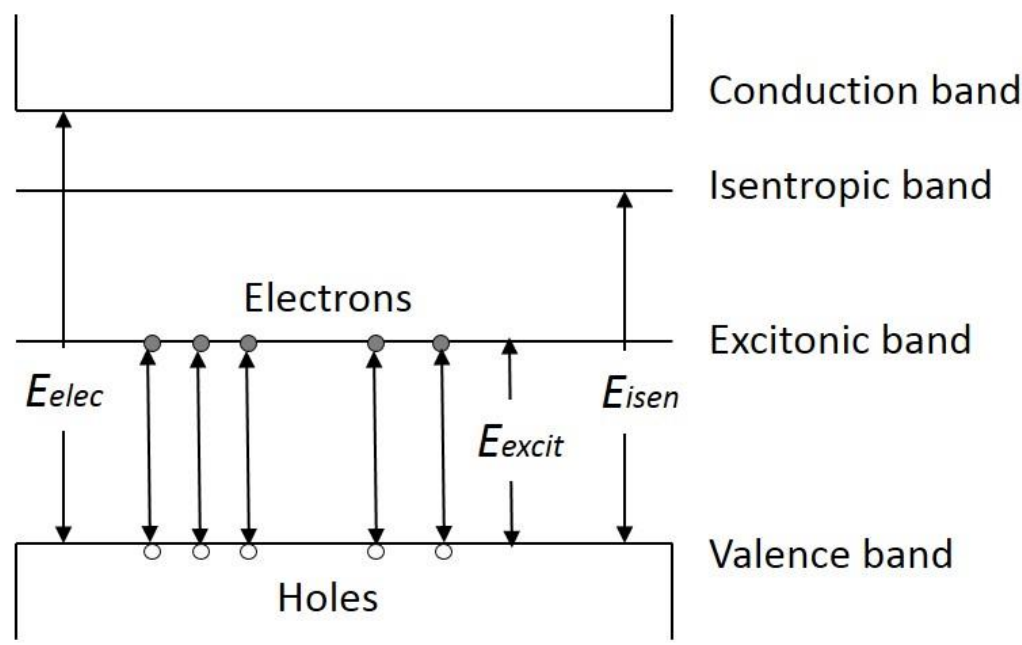

Figure 1.23: Schematic representation of the energy levels of optical glasses from [66]..

It has been shown quite recently that a rigorous description of the thermal behavior may be obtained in a much more comprehensive manner [57]. Despite being said as deduced from a "semi-empirical" model, Sellmeier equations have been proved for long to give the best representation of the refractive index dispersion of any optical material all over its transparency window. Thus, in accordance with other authors [67], we would recommend to fit recorded refractive index data by using such formulation. Direct measurements of the dispersion $n(\lambda)$ are usually obtained from various non 
coherent impinging light sources and at so-called "room temperature", say $T_{0}$, by employing prism methods performed in the minimum of deviation configuration. From a literature survey it can be estimated that mean values of best affordable accuracies lie around $10^{-5}$ in the visible spectral range and a few in $10^{-4}$ in the mid-IR region and for samples of large aperture. At a fixed temperature $T_{0}$, the data may be fitted to the simple Sellmeier's relationship 1.47 mentioned in § 1.1.2.2 and written in the slightly modified form:

$$
\left.n^{2}(\lambda)\right|_{T_{0}}=A+\sum_{i=1}^{l} \frac{B_{i}}{\lambda^{2}-\lambda_{i}^{2}}
$$

$A$ is a constant and the summation extends over a finite number $l$ of oscillators $i$ resonant at wavelengths $\lambda_{i}=\frac{2 \pi c}{\omega_{i}}, c$ being the speed of light. Parameters $B_{i}$ depends on the strength of each oscillator that is to be taken into account to achieve best fitting procedure. Two sets of terms are introduced formally to account for UV and IR cutting edges, beyond which the material becomes opaque.

Now, considering that formula 1.132 is also temperature dependent and taking then the logarithmic expression we get:

$$
\ln [n(\lambda, T)]=\frac{1}{2} \ln \left[A(T)\left(1+\sum_{i=1}^{l} \frac{\left(B_{i} / A\right)(T)}{\lambda^{2}-\lambda_{i}^{2}(T)}\right)\right]
$$

Over the range of transparency, far enough from cutting edges, the dispersion is represented by the summation term which figures only slight and monotonically wavelength-dependent deviations from the mean value $A(T)$, that is:

$$
\sum_{i=1}^{l} \frac{\left(B_{i} / A\right)(T)}{\lambda^{2}-\lambda_{i}^{2}(T)}<<1
$$

Having defined the normalized thermo-optic coefficient $\beta_{N T O C}=\frac{1}{n} \frac{d n}{d T}$, we may write:

$$
\beta_{N T O C}(\lambda, T)=\frac{1}{\left.2 n_{\lambda_{i}}^{2}\right|_{T_{0}}}\left[\frac{d A(T)}{d T}+\sum_{i=1}^{l} \frac{1}{\left[\lambda^{2}-\lambda_{i}^{2}(T)\right]} \frac{d B_{i}(T)}{d T}+\sum_{i=1}^{l} \frac{2 B_{i}(T) \lambda_{i}(T)}{\left[\lambda^{2}-\lambda_{i}^{2}(T)\right]^{2}} \frac{d \lambda_{i}(T)}{d T}\right]
$$

Now $\beta_{N T O C}(T)$ data may always be accurately fitted to a power series of temperature down to better than $10^{-7} \mathrm{~K}^{-1}$ accuracy:

$$
\beta_{\text {NTOC }}(\lambda, T)=c_{0}(\lambda)+c_{1}(\lambda) T+\ldots . .+c_{m}(\lambda) T^{m}=\sum_{0}^{m} c_{j}(\lambda) T^{j}
$$

Determined experimentally, the $c_{j}$ 's must be consistent with equation (1.135) and will therefore be written according to: 


$$
c_{j}(\lambda)=\frac{1}{\left.2 n_{\lambda}^{2}\right|_{T_{0}}}\left[X_{1}+\sum_{i=1}^{l} \frac{1}{\left[\lambda^{2}-\lambda_{i}^{2}(T)\right]^{\prime}} X_{i}+\sum_{i=1}^{l} \frac{2 B_{i}(T) \lambda_{i}(T)}{\left[\lambda^{2}-\lambda_{i}^{2}(T)\right]^{2}} X_{i}^{\prime}\right]
$$

obtained from (1.135) by putting $X_{1}=\frac{d A(T)}{d T}, X_{i}=\frac{d B_{i}(T)}{d T}$ and $X_{i}^{\prime}=\frac{d \lambda_{i}(T)}{d T}$

Equation (1.137) represents a set of $(2 l+1)$ linear equations with unknown parameters $X_{1}, X_{i}$ and $X_{i}^{\prime}$ , which can readily be solved by using a simple vectorial formalism if NTOC data are obtained at a number of $(2 l+1)$ laser wavelengths suitably chosen in the transparency window of the material. A set of $(2 l+1)$ experimental values of the $c_{j}$ 's will allow to determine the temperature-dependent dispersion formula (1.132), which is obtained by integrating equation (1.136) and knowing the dispersion equation at room temperature $T_{0}$. This gives:

$$
n(\lambda, T)=n\left(\lambda, T_{0}\right) \exp \left[\sum_{j=0}^{m} \frac{c_{j}(\lambda)}{j+1}\left(T^{j+1}-T_{0}^{j+1}\right)\right]
$$

The refractive index could as well be written in a Sellmeier like formulation:

$$
n^{2}(\lambda, T)=(A+\Delta A)+\sum_{i=1}^{l} \frac{B_{i}+\Delta B_{i}}{\lambda^{2}-\left[\lambda_{i}^{2}+\Delta\left(\lambda_{i}^{2}\right)\right]}
$$

where $\Delta A, \Delta B_{i}$ and $\Delta\left(\lambda_{i}^{2}\right)$ are functions of temperature $T$ and obtained by integrating equations (1.138):

$$
\Delta A=\int_{T_{0}}^{T} X_{1} d T, \Delta B_{i}=\int_{T_{0}}^{T} X_{i} d T \text { and } \Delta\left(\lambda_{i}^{2}\right)=\int_{T_{0}}^{T} X_{i}^{\prime} d T
$$

Expression 1.140 is reminiscent of the pure empirical ones proposed in the case of $\mathrm{LiNbO}_{3}$ [68-69] and $\mathrm{MgO}$ doped $\mathrm{LiTaO}_{3}$ crystals [70].

\subsubsection{Accuracy: temperature dependence of an $\mathrm{ONL}$ interaction in $\mathrm{RbTiOPO}_{4}$}

If $\sigma_{\alpha_{L}}$ and $\sigma_{\gamma_{N T C O P}}$ are the uncertainties associated to measurements of thermal expansion and changes in optical length of a sample of length $L$ examined over a temperature interval $\Delta T$, the uncertainty on NTOC is given by:

$$
\sigma_{\beta_{N T O C}}=\sigma_{\alpha_{L}}+\sigma_{\gamma_{N T C O P}}=\left(\alpha_{L}+\gamma_{N T C O P}\right)\left[\frac{\sigma_{L}}{L}+\frac{\sigma_{\Delta T}}{\Delta T}\right]+\gamma_{N T C O P} \frac{\sigma_{n}}{n}+\frac{1}{L} \frac{\sigma_{\Delta L}}{\Delta T}+\frac{1}{n L} \frac{\sigma_{\Delta(n L)}}{\Delta T}
$$


From experience, estimates of all sources of uncertainty lead us to conclude that the order of magnitude of $\sigma_{\beta_{\text {NTOC }}}$ is of a few in $10^{-6} \mathrm{~K}^{-1}$ in most cases. Notice that such assertion comes out from linear optics measurements and "classical" uncertainty calculation (i. e. without any statistical consideration). However, confirmation of this has been clearly evidenced through a nonlinear optics experimentation that is described hereafter.

The temperature dependence of the second harmonic generation (SHG) $1.0642 \mu \mathrm{m} \rightarrow 0.532 \mu \mathrm{m}$ was studied in the case of an $\mathrm{RbTiOPO}_{4}$ (RTP) single crystal [71]. Apart from the requirement of an accurate knowledge of the dispersion equations at a fixed temperature $T_{0}$, it is known that any temperature variation of the crystal will alter the doubling efficiency. In such critical process, refractive indices modifications are to be characterized at least down to the fifth decimal place. RTP belongs to the anisotropic orthorhombic $\mathrm{mm} 2$ symmetry class; therefore three Sellmeier equations analogous to formula (1.132) have to be considered to characterize the dispersion of the refractive indices $n_{x}, n_{y}$ and $n_{z}$ along the three principal dielectric axes $\mathrm{Ox}, \mathrm{Oy}$ and $\mathrm{Oz}$ of the crystal, respectively. Interferometric measurements of the corresponding three principal thermal expansion coefficients of RTP have been obtained from $-30^{\circ} \mathrm{C}$ to $+130^{\circ} \mathrm{C}$ by using a frequency stabilized He-Ne laser. The NTOC's were determined over the same temperature interval from measurements of normalized coefficients of changes in optical path performed at four CW laser wavelengths and with appropriate polarization direction of the light with respect to the $X, Y$ and $Z$ axes. Single mode $C W$ lasers were used: an argon ion tuned at $0.4578 \mu \mathrm{m}$, two He-Ne emitting respectively at 0.6328 and $3.39 \mu \mathrm{m}$ and a Nd:YAG at $1.0642 \mu \mathrm{m}$. A linear behavior of $\alpha_{L}(T)$ coefficients was observed, with $\alpha_{z}<0$, while the NTOC's exhibit a quadratic one; they are given in fig (1.24) for illustration.

Type II eo-e Second Harmonic Generation (SHG) is a nonlinear optical interaction where an extraordinary photon combines with an ordinary one at the fundamental wavelength $\lambda$ to give an extraordinary wave at $\lambda / 2$. The SHG $1.0642 \mu \mathrm{m} \rightarrow 0.5321 \mu \mathrm{m}$ is governed by the phase mismatch $\Delta k$ written as:

$$
\Delta k(T)=\frac{2 \pi}{\lambda}\left[2 n^{\lambda / 2}(T)-\left(n^{\lambda}(T)+n_{z}^{\lambda}(T)\right)\right]
$$

$n^{\lambda / 2}(T)$ and both $n^{\lambda}(T), n_{z}^{\lambda}(T)$ are the temperature and orientation-dependent refractive indices at the second harmonic and fundamental waves, respectively. In the $(X, Y)$ crystallographic plane and for an impinging internal angle $\varphi$ referred to the $X$ axis, the refractive index $n$ is given by:

$$
\frac{1}{n^{2}(\varphi)}=\frac{\cos ^{2} \varphi}{n_{y}^{2}}+\frac{\sin ^{2} \varphi}{n_{x}^{2}} \text {. }
$$




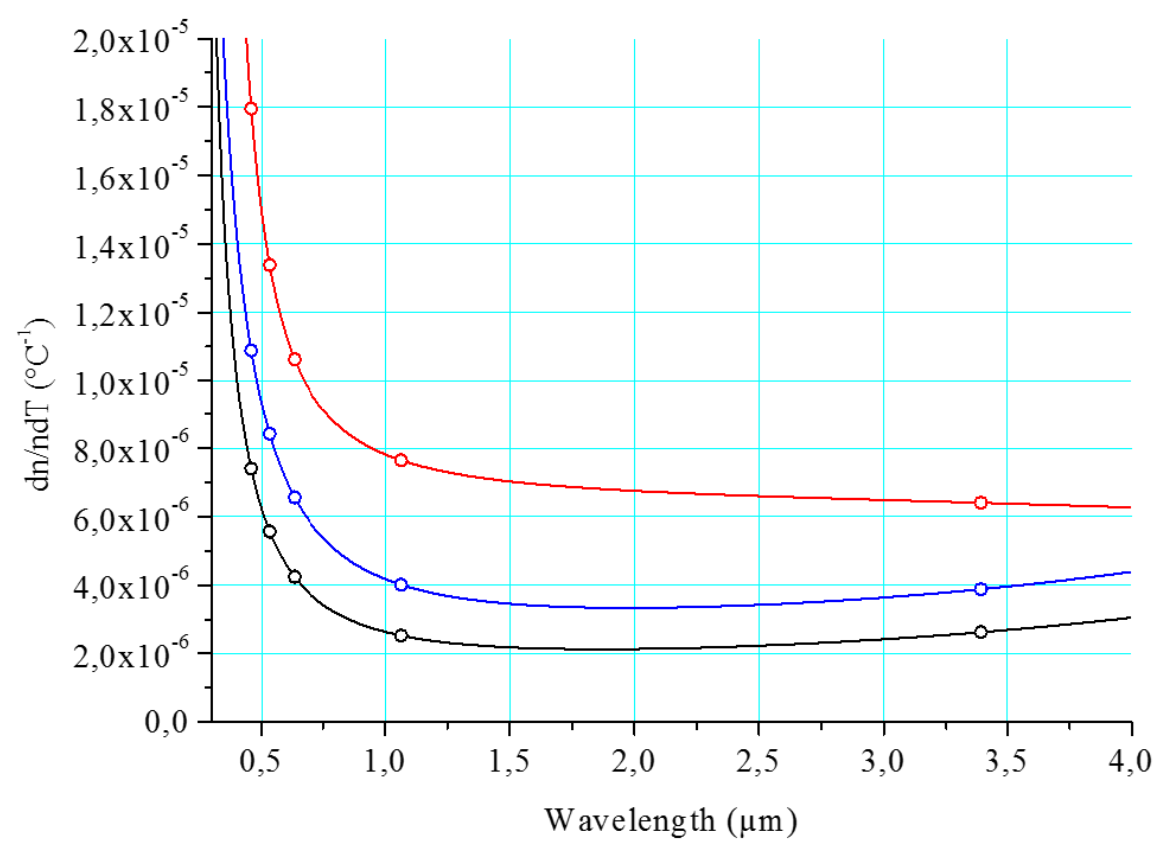

Figure 1.24: NTOC's dispersion obtained for RTP at room temperature $\left(20^{\circ} \mathrm{C}\right)$ and starting from refractive index dispersion given in ref [71]. Open circles correspond to experimental data and full lines to fitting results; black, blue and red colors refer to $X, Y$ and $Z$ polarizations, respectively.

Phase matched interaction corresponds to $\Delta k=0$, which is for:

$$
n^{\lambda / 2}(T)=\frac{1}{2}\left[n^{\lambda}(T)+n_{z}^{\lambda}(T)\right] \text { (1.145) }
$$

In biaxial RTP and starting from the dispersion equations given in ref [71], this occurs at $T_{0}=20^{\circ} \mathrm{C}$ for phase-matching angle $\varphi_{\mathrm{pm}}=\varphi_{0}=55.82^{\circ}$. The temperature dependence of $\varphi_{\mathrm{pm}}$ is determined by solving numerically formula (1.145) and by using the $n(T)$ values derived from NTOC's measurements following the procedure described in section 1.3.3. The result is drawn as full curve in figure 1.25 which gives the predicted evolution of the phase-matching angle $\varphi_{\mathrm{pm}}$ from $-20^{\circ} \mathrm{C}$ up to $230^{\circ} \mathrm{C}$. 


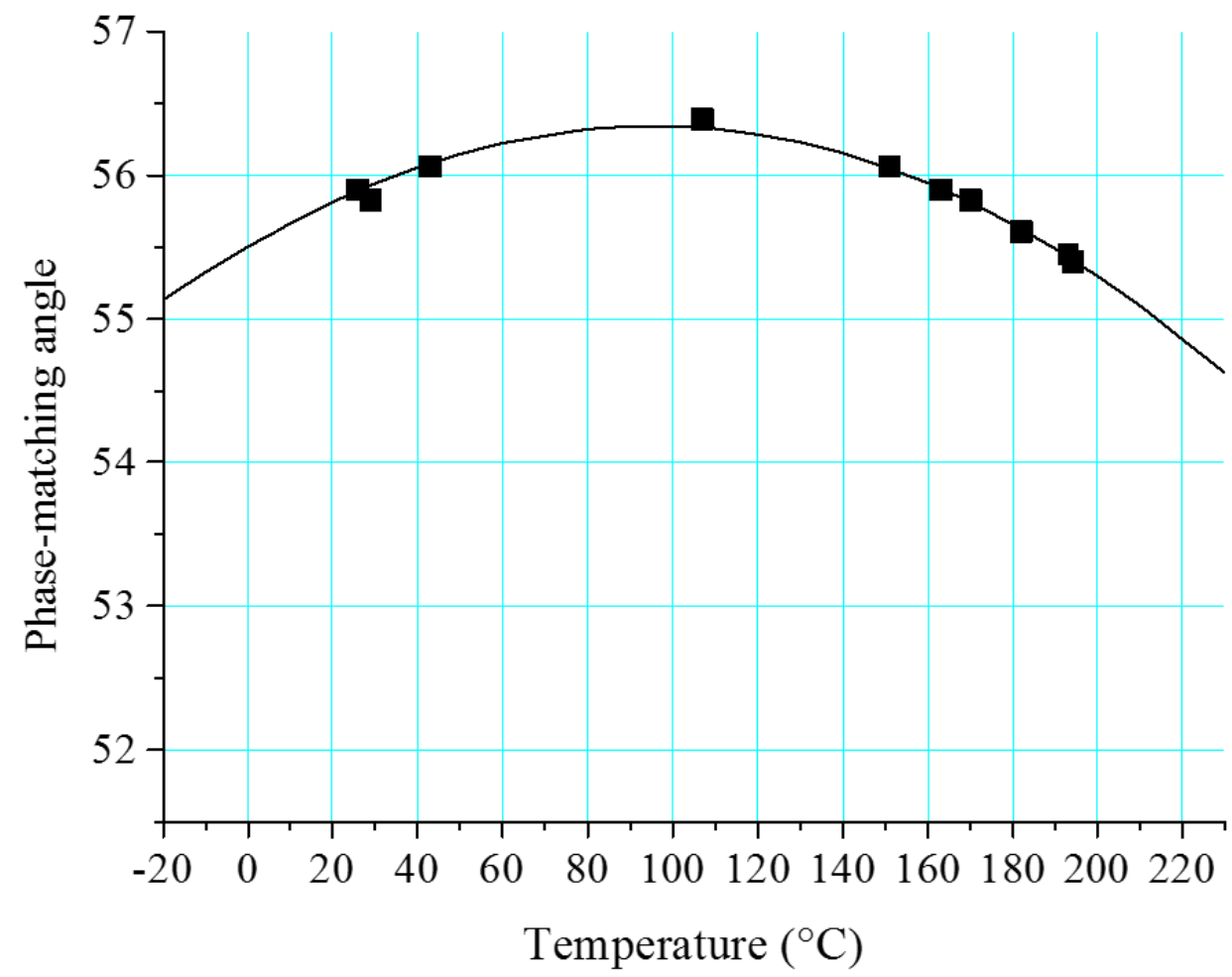

Figure 1.25: Predicting the temperature dependence of the phase-matching angle in the $(X, Y)$ plane of RTP for the SHG of Nd:YAG lasers. Full curve: theory, from formula (1.145); the black squares correspond to recorded experimental maxima of efficiency.

\subsection{Spectrophotometric determination of refractive indices}

Spectrophotometers are widely used in industry and in research laboratories for determining the spectral transmittance, reflectance and absorptance of optical materials. This section starts by recalling some of their basic characteristics and methods, estimated to be useful for the understanding of the way they can be used for the measurement of the refractive index and extinction coefficient of bulk and coated samples. The following cases are then successively covered: bulk materials, homogeneous thin films, inhomogeneous thin films, metallic films on transparent media, and optical constant determination by the bilayer "metal-dielectric" method.

\subsubsection{Some useful properties of spectrophotometers}

This sub-section deals with some of the basic characteristics of spectrophotometers, starting by a description of their main constituents. It then defines their "coherence length" and the measurable 
quantities that are of particular interest for characterizing the refractive indices of films and of coated or uncoated samples. The "V-W" procedure being very commonly used, its configuration is presented here, along with some typical spectral results in transmission and reflection.

\subsubsection{Structure and main components of a spectrophotometer}

The main components of standard spectrophotometers are the following:

a) The light source, which is chosen in connection with the spectral domain of interest. In the UV, deuterium lamps operate from 195 to $380 \mathrm{~nm}$; in the visible and near IR, the sources are usually of the tungsten halogen type (from 320 to $1000 \mathrm{~nm}$ ) or Xenon lamps (from 190 to 1100 $\mathrm{nm})$, and blackbodies are used to cover the IR.

b) A monochromator, in charge of filtering the input light inside a narrow bandwidth (or spectral resolution) $\Delta \lambda$ around some wavelength of interest $\lambda$ and of scanning (or not) this selection over the spectral domain of investigation.

c) A telescopic goniometer in charge of adjusting the size, the direction, and in some cases, the polarization of the beam of light that illuminates the sample. This component monitors the angle of incidence of the beam upon the optical sample under test.

d) The sample to be characterized, generally shaped as a plane parallel plate. In most spectrophotometers the test sample is characterized by comparing the fluxes collected from it with those obtained in the same configuration from a reference sample (specially designed for the task). Hence, the test and reference samples are alternately illuminated in similar conditions.

e) The detection and its associated signal processing electronics, that convert the different (reflected, transmitted, scattered) collected amounts of flux into recordable electric signals.

Hence, it is important to notice that most of the values delivered by a spectrophotometer of optical parameters, such as transmittance, or reflectance, are dimensionless and are the ratios between the same quantities (fluxes) respectively measured from the test and reference channels_under similar configurations.

The reference sample (or channel) plays an important role since it is used as a scale and defines the so-called "baseline" of the instrument. 


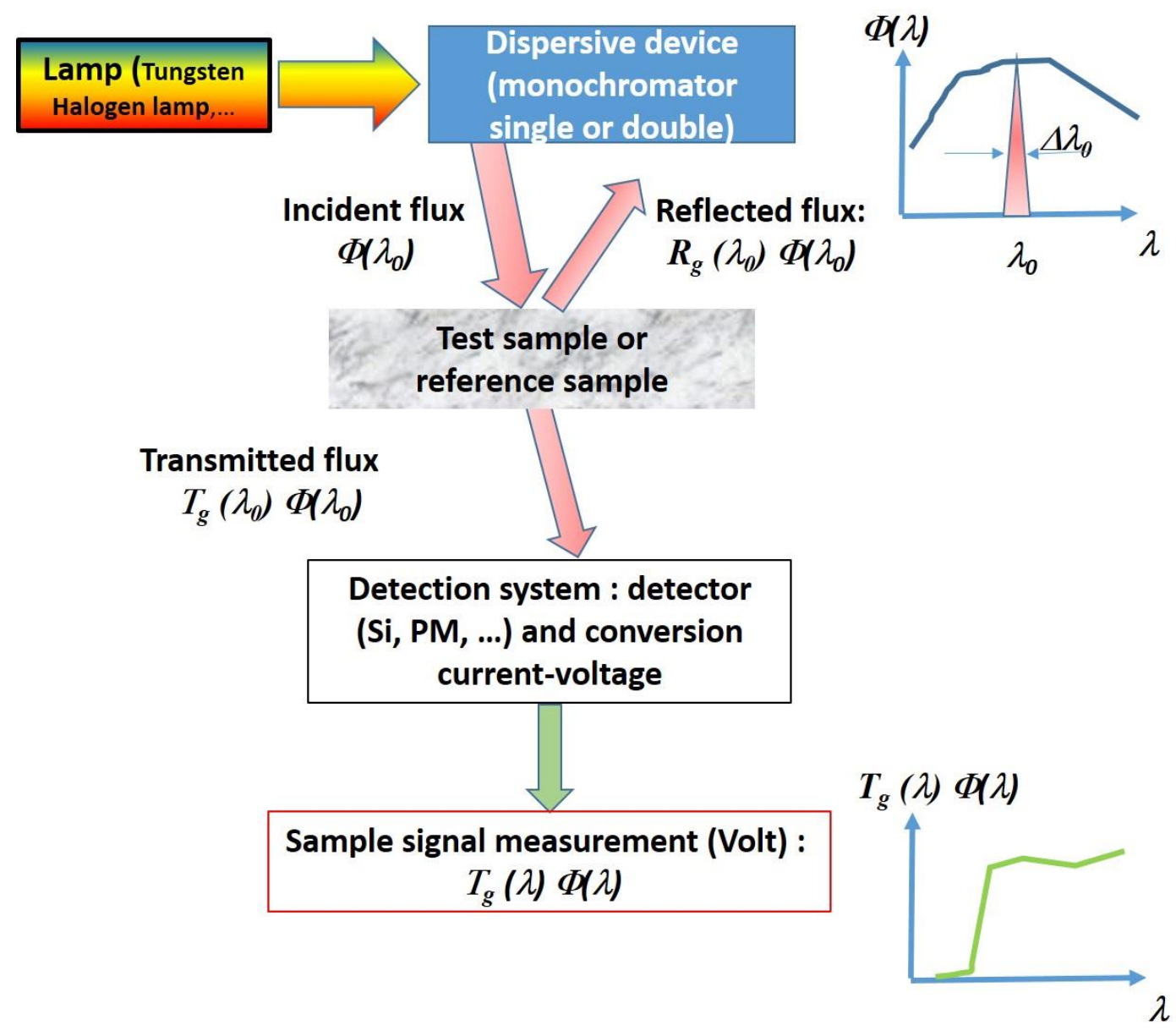

Figure 1.26: Operating principle of a spectrophotometer used for measuring the transmitted flux of a sample defined by $T_{g}(\lambda) \Phi(\lambda)$, where $T_{g}(\lambda)$ is the global spectral transmittance (respectively global spectral reflectance $\left.R_{g}(\lambda)\right)$ and $\Phi(\lambda)$ the incident flux. .

\subsubsection{Coherence length of the spectrophotometer}

The coherence length $\left(\mathrm{L}_{\mathrm{c}}\right)$ of the spectrophotometer is defined by equation 1.146 below:

$$
L_{c}=\frac{\lambda_{0}^{2}}{\delta \lambda_{0}}=\frac{1}{\delta \text { i\& }}(1.146)
$$

Where $\lambda_{0}$ is the mean wavelength of the spectrally filtered beam, and $\delta \lambda_{0}$ or $\delta 1 \&$ its full widths at half maximum (FWHM), expressed in wavelength $(\delta \lambda)(\mu \mathrm{m}$ or $\mathrm{nm})$ or in wavenumber $\left(\delta \&\left(\mathrm{~cm}^{-1}\right)\right.$ units. The value of this coherence length is of prime importance because the shape of the output spectral data (such as spectral reflectance or transmittance) from the spectrometer depends upon the ratio between the sample thickness and $L_{\mathrm{c}}$. As will be shown in subsection 1.4.1.3, the incident beam gets separated into two or more beamlets that recombine on the sensitive area of the detector after bouncing back and forth from the sample boundaries. If the corresponding optical path difference (OPD $\approx 2 n d$ for quasi-normal incidence) is much less than the coherence length, these interfering waves retain some mutual coherence, and their recombination gives rise to interference phenomena, 
converted as ripples on the spectral graphs. The lower the ratio $\left(\mathrm{OPD} / L_{\mathrm{c}}\right)$, the higher the mutual coherence beam, and the higher the ripple contrast. Conversely, if $O P D \geq L_{\mathrm{c}}$, the interfering beams are un-correlated. Their recombination does not give rise to static interferences, and the corresponding spectral graphs are smooth.

Figure 1.27 shows the graph, over the $900-940 \mathrm{~cm}^{-1}$ domain, of the spectral transmittance of a $514 \mu \mathrm{m}$ thick plate of polycrystalline isotropic diamond $\left(n_{\text {diamond }} \approx 4\right)$ obtained from a Bomem Fourier Transform Infrared spectrometer, of spectral resolution $\delta \& \&=0.04 \mathrm{~cm}^{-1}$. In this example, $L_{c}=1 / \delta 1 \&=25 \mathrm{~cm}$ $>2 n_{\text {diamond }} d \approx 4 \mathrm{~mm}$, which explains the rather high contrast $\left(C=\left(T_{\max }-T_{\min }\right) /\left(T_{\max }+T_{\min }\right) \approx 25 \%\right)$ of the ripples. The fringe spacing, or free spectral range $\Delta \&$ (i.e. the difference between two adjacent wavenumbers of identical state of interference), given by equation (1.146b) equals about $4 \mathrm{~cm}^{-1}$ and the resulting number of oscillations (ratio between $40 \mathrm{~cm}^{-1}$, the covered spectral range, and $4 \mathrm{~cm}^{-1}$, the free spectral range), is around 10, as confirmed on Figure $1.27[5,73]$. The black curve comes out from experimentation and the red one corresponds to the theoretical fit obtained by using a one pole Cauchy's model for the dispersion of the refractive index $[5,73]$.

In practice, it is advisable to choose a substrate of appropriate thickness (i.e. $>>\mathrm{L}_{c}$ ) with respect to the resolution $\delta \lambda_{0}$ in order to better highlight the interference features specific to multilayer.

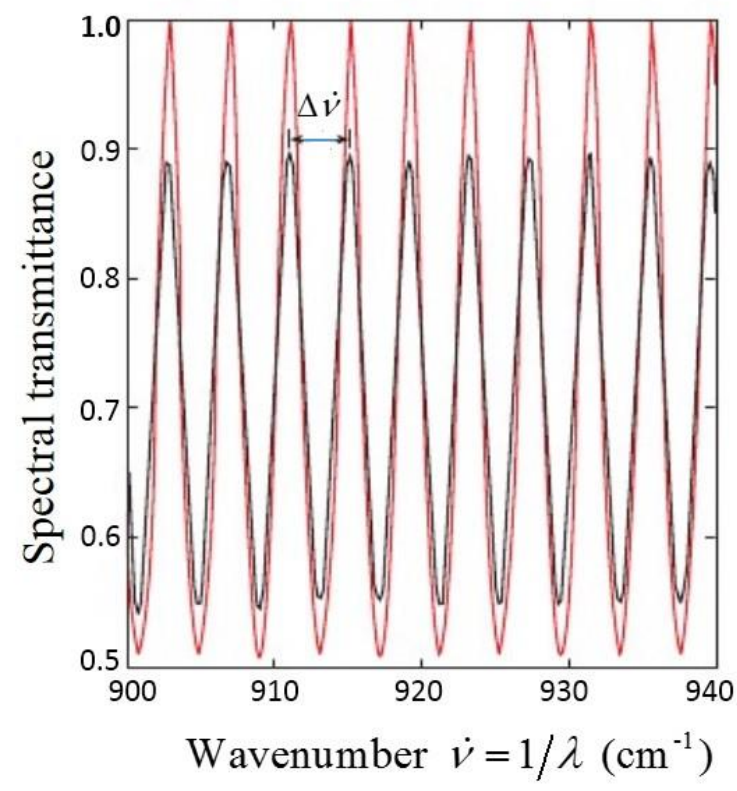

Figure 1.27: Spectral transmisttance of a $514 \mu \mathrm{m}$ thick plate of diamond from $900 \mathrm{~cm}^{-1}$ to $940 \mathrm{~cm}^{-1} . \Delta \mathrm{l} \&$ is the nominal free spectral range (i.e. fringe spacing) in the explored spectral interval from [73].

\subsubsection{Measurable quantities}

As mentioned in sub-section 1.4.1.1, the sample under test (either bare or coated substrate) transmits, reflects and/or absorbs parts of the incident pencil of light which is spectrally filtered at some 
wavelength $\lambda$ (scanned over the spectral domain of interest), and the spectrophotometer outputs are the detector signals in response to the corresponding collected fluxes. This subsection briefly describes the methodology that allows deriving the optical parameters of the sample, i.e. the refractive index and extinction coefficient of the substrate and of its coating, as well as the film thickness, from the measured values of the global spectral reflectance, denoted $R_{g}(\lambda)$ and of the global spectral transmittance, denoted $T_{g}(\lambda)$, of the sample.

For this purpose, the sample is generally shaped as a plane parallel plate of high quality, with polished surfaces, in order to avoid any deviation or other type of modification of the reflected and transmitted beam spots onto the sensitive area of the detector and hence to ensure an optimized detection of all fluxes of interest. Figure 1.28 shows the case of a non-absorbing sample, of refractive index $n_{s}(\lambda)$ and thickness $d_{s}$, illuminated under quasi-normal incidence; its upper boundary is only coated over one half of its surface by the thin film to characterize, and the lower one is not coated at all. The surrounding medium is supposed to be air, of refractive index $n_{0}(\lambda) \approx 1$.

In usual spectrophotometric experiments, three configurations of sample illumination are necessary in order to provide the expected information, according to Figure 1.28a: in configuration 1, on the left hand side, the downward propagating incident beam hits the bare part of the sample (both interfaces of the substrate are uncoated). At center, the sample is translated in such a way that the same downward propagating incident beam firstly hits the coated part of the upper surface of the sample and then its uncoated rear surface. At the right hand side of the figure1.28a, the incident beam propagates upwards and firstly hits the (uncoated) lower surface of the sample and then the coated surface (in practice, the sample is turned upside down).

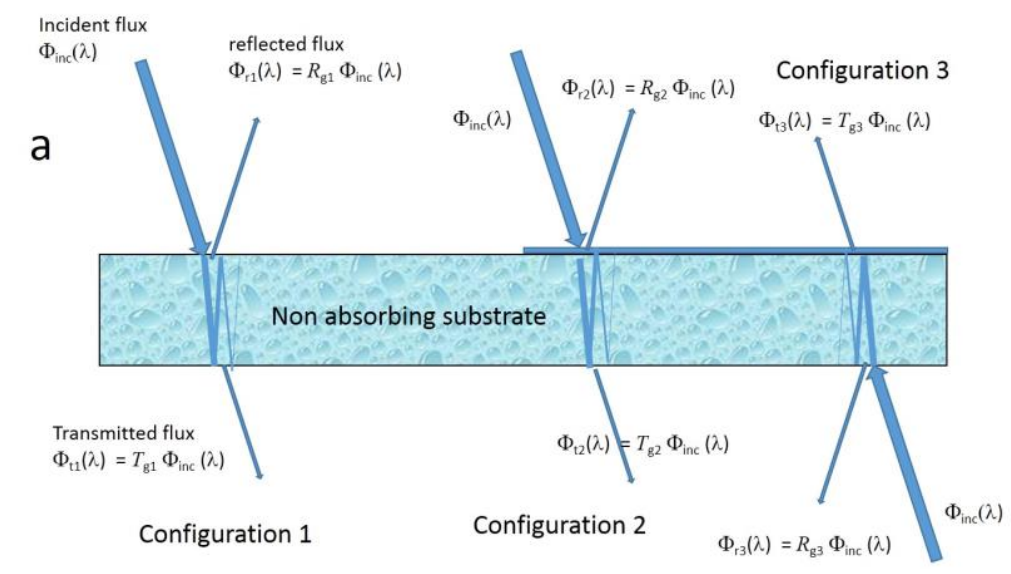

b

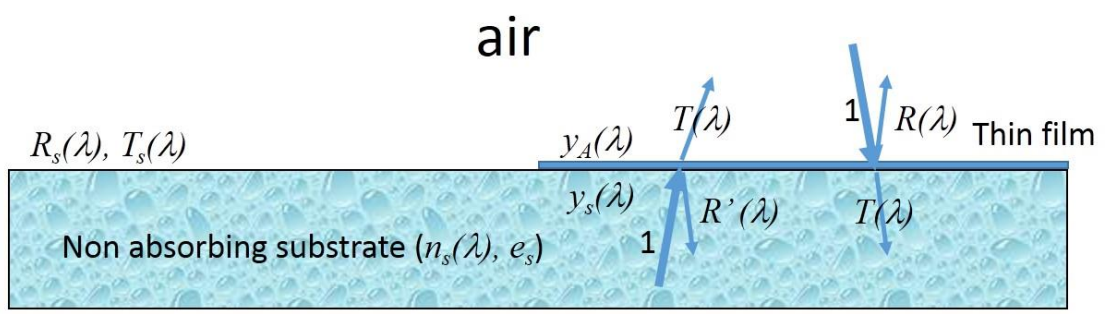

$R_{s}(\lambda), T_{s}(\lambda)$

air 
Figure 1.28: Typical illuminating configurations of the sample with corresponding measured quantities (fluxes $\Phi)(1.28 a)$, and parameters of the sample that can be extracted from these measurements (1.28b)

Let the (unknown) spectral reflectance and transmittance of the film (as deposited on the substrate) be denoted $R(\lambda)$ and $T(\lambda)$ for a downward propagation of the light (i.e. for rays incident from air to film), and $R^{\prime}(\lambda)$ and $T^{\prime}(\lambda)(=T(\lambda))$ for an upward direction of propagation (i.e. for an incident beam propagating from the substrate to the film); the spectral reflectance and transmittance of each uncoated boundary are respectively denoted $R_{\mathrm{s}}(\lambda)$ and $T_{s}(\lambda)$ (Figure $\left.1.28 \mathrm{~b}\right)[5,7,74,75]$.

Without getting into detailed calculations, one shows that the flux that is reflected by the sample is the summation of the flux reflected from the first encountered interface and the multiple ones carried by the beams that propagate inside the substrate and bounce back and forth from its interfaces (coated or not). This summation of fluxes (and not of field amplitudes is justified by the fact that the coherence length of the incident beam is supposed to be much less than the sample thickness. Similarly, the flux that is transmitted by the sample is the summation of the directly transmitted flux and the multiple contributions that have been bouncing back and forth inside the substrate. Table 1.5 gives the theoretical expressions of the global spectral reflectance $R_{g}(\lambda)$ and global transmittance $T_{g}(\lambda)$ of the sample corresponding to the reflected and transmitted fluxes as measured in the three configurations of Figure 1.28a:

\begin{tabular}{|c|c|c|}
\hline Configuration & Spectral reflectance & Spectral transmittance \\
\hline 1 & $R_{g 1}(\lambda)=\frac{2 R_{s}(\lambda)}{1+R_{s}(\lambda)}(1.147)$ & $T_{g 1}(\lambda)=\frac{1-R_{s}(\lambda)}{1+R_{s}(\lambda)}(1.150)$ \\
\hline 2 & $R_{g 2}(\lambda)=R(\lambda)+\frac{T^{2}(\lambda) R_{s}(\lambda)}{1-R_{s}(\lambda) R^{\prime}(\lambda)}(1.148)$ & $T_{g 2}(\lambda)=\frac{T(\lambda)\left(1-R_{s}(\lambda)\right)}{1-R_{s}(\lambda) R^{\prime}(\lambda)}(1.151)$ \\
\hline 3 & $R_{g 3}(\lambda)=R_{s}(\lambda)+\frac{T_{s}^{2}(\lambda) R^{\prime}(\lambda)}{1-R_{s}(\lambda) R^{\prime}(\lambda)}(1.149)$ & $T_{g 3}(\lambda)=\frac{T(\lambda)\left(1-R_{s}(\lambda)\right)}{1-R_{s}(\lambda) R^{\prime}(\lambda)}(1.151)$ \\
\hline
\end{tabular}

Table 1.5: Computed values of global spectral reflectance and transmittance for the three illuminating configurations of Figure 1.28 (case of a non absorbing bulk material)

The equivalence between the respective ratios between the experimentally measured reflected or transmitted fluxes and the incident flux, and the corresponding expressions of the reflectance or transmittance of the sample as expressed in Table 1.5 yield the optical parameters of the sample:

For a transparent substrate, the spectral reflectance $R_{s}(\lambda)$ and transmittance $T_{s}(\lambda)$ of each uncoated boundary, given by the Fresnel formulas under normal incidence, lead to the refractive index $n_{s}(\lambda)$ of the bulk material: 


$$
R_{s}(\lambda)=\left(\frac{n_{s}(\lambda)-n_{0}(\lambda)}{n_{s}(\lambda)+n_{0}(\lambda)}\right)^{2} \quad \text { and } \quad T_{s}(\lambda)=1-R_{s}(\lambda)
$$

The experimental verification of the equality at the right hand side of equation (1.152) confirms the fact that the material of the substrate is transparent.

At each wavelength, if the optical coating and the substrate are neither absorbing nor diffuse, the energy conservation law may be written for each configuration in the following manner:

$$
T_{g}(\lambda)+R_{g}(\lambda)=1(1.153)
$$

If there are light losses along the optical path inside the sample, due to absorption and/or scattering, the energy conservation law becomes:

$$
T_{g 1}(\lambda)+R_{g 1}(\lambda)+\Lambda_{1}(\lambda)=T_{g 2}(\lambda)+R_{g 2}(\lambda)+\Lambda_{2}(\lambda)=T_{g 3}(\lambda)+R_{g 3}(\lambda)+\Lambda_{3}(\lambda)=1
$$

Where the $\Lambda$ 's are the spectral loss coefficients including the absorptance $(A)$ and scattering (TIS, defined in subsection 1.4.1.4), with: $\Lambda(\lambda)=A(\lambda)+T I S(\lambda)$

If scattering is negligible, for each wavelength, the values of the three unknown $R(\lambda), R^{\prime}(\lambda)$ and $T(\lambda)$ can be extracted from the three experimental values of $R_{\mathrm{g} 2}(\lambda)$, of $R_{\mathrm{g} 3}(\lambda)$ and of $T_{\mathrm{g} 2}(\lambda)$, and hence allow to compute the film admittances $Y_{A}(\lambda)$ and $Y_{s}(\lambda)$, defined in subsection 1.1.6.2 and considered here at the respective film interfaces with air and with the substrate by the following expressions :

$$
R(\lambda)=\left|\frac{n_{0}(\lambda)-Y_{A}(\lambda)}{n_{0}(\lambda)+Y_{A}(\lambda)}\right|^{2}(1.155) \text { and } R^{\prime}(\lambda)=\left|\frac{n_{s}(\lambda)-Y_{s}(\lambda)}{n_{s}(\lambda)+Y_{s}(\lambda)}\right|^{2}
$$

It must be noted that the computation of $R(\lambda), R^{\prime}(\lambda)$ and $T(\lambda)$ differs from that of $R_{\mathrm{g}}(\lambda)$ and $T_{\mathrm{g}}(\lambda)$ because the evaluation of $Y_{A}(\lambda)$ and $Y_{S}(\lambda)$ results from the vector sum of the field amplitudes of the beams bouncing back and forth from the boundaries of the coating, since the film thickness is supposed to be much smaller than the coherence length of the incident light.

At wavelengths for which the substrate is slightly absorbing $\left(\kappa_{s} \neq 0\right)$, these parameters may be evaluated in a similar manner, at the condition, though, that the substrate thickness $d_{s}$ be taken into account. At this condition, the expressions of the measured transmittance and reflectance become:

$$
\begin{gathered}
T_{g 2}(\lambda)=\frac{T(\lambda) T_{s}(\lambda) \exp \left(-\frac{\alpha_{s} d_{s}}{2}\right)}{1-R_{s}(\lambda) R^{\prime}(\lambda) \exp \left(-\alpha_{s} d_{s}\right)} \\
R_{g 2}(\lambda)=R(\lambda)+\frac{T^{2}(\lambda) R_{s}(\lambda) \exp \left(-\alpha_{s} d_{s}\right)}{1-R_{s}(\lambda) R^{\prime}(\lambda) \exp \left(-\alpha_{s} d_{s}\right)} \\
R_{g 3}(\lambda)=R_{s}(\lambda)+\frac{T_{s}^{2}(\lambda) R^{\prime}(\lambda) \exp \left(-\alpha_{s} d_{s}\right)}{1-R_{s}(\lambda) R^{\prime}(\lambda) \exp \left(-\alpha_{s} d_{s}\right)}
\end{gathered}
$$


where $\alpha_{s}=\frac{4 \pi}{\lambda} \kappa_{s}$ is the absorption coefficient.

\subsubsection{The " $V$ " and " $W$ " configurations}

In order to correctly measure the above-mentioned quantities, it is essential to use a unique and stable source and the same detector to collect the flux. It is also important that the configurations for the test and the reference samples be as similar as possible, hence the interest of the so-called V-W configuration which is described in this sub-section.

Figures 1.29a and 1.29b show the optical set-up of this method that can be modified from its " $V$ » configuration for measuring the spectral transmittance of the sample into its " $W$ " configuration for the spectral reflectance, by means of a retractable mirror, that stops the transmitted beam and redirects the reflected beam towards the final mirror without any modification in the external path. The angle of incidence is generally around $8^{\circ}$.

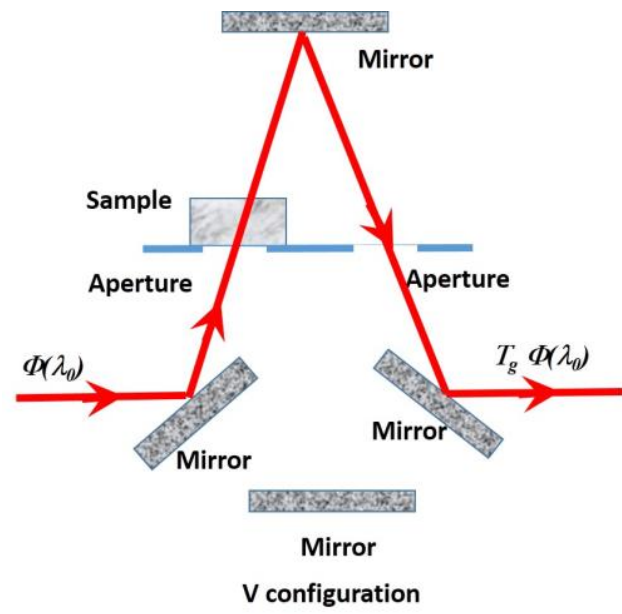

Figure 1. 29a: "V"-Configuration

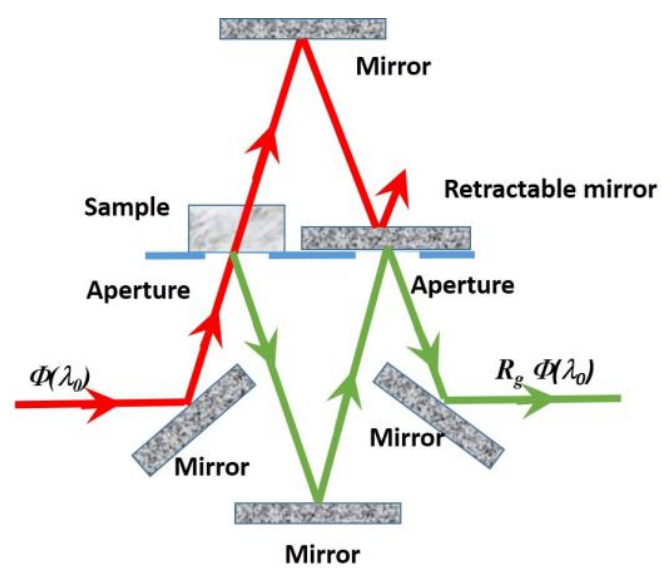

W configuration

Figure 1. 29b: "W"-Configuration

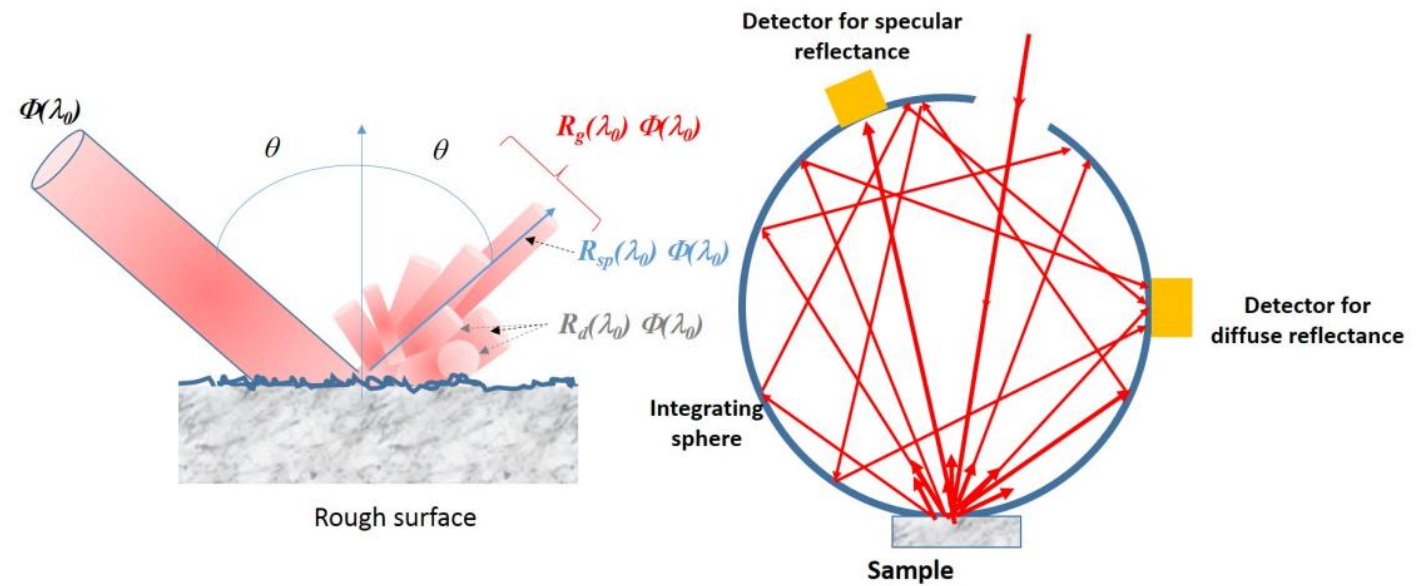


Figure 1.29 c: Integrating sphere used for measuring the specular and diffuse reflectance of a rough surface.

As shown on Figure 1.29c, the fraction $R_{\mathrm{sp}}(\lambda)$ of the incident light reflected from a surface propagates along the direction specified by the Snell-Descartes law and corresponds to the specular reflection of that surface. The other fraction $R_{\mathrm{d}}(\lambda)$ of the reflected light, which is scattered all over the other directions in space corresponds to the diffuse reflection of the surface. Consequently, the global reflectance $R_{\mathrm{g}}(\lambda)$ of the surface is the sum of these two contributions:

$$
R_{g}(\lambda)=R_{s p}(\lambda)+R_{d}(\lambda)(1.160)
$$

It can be shown that scattering of light originates from the surface roughness: if the surface is modelled as a Gaussian random surface of root mean square $(\mathrm{rms})$ roughness $\sigma_{r}$ (supposed to be small compared to the wavelength of the incident beam), its specular reflectance is related to its global reflectance and to the $r m s$ roughness of the surface by the following relationship [76-78]:

$$
R_{s p}(\lambda)=R_{g}(\lambda) \exp \left[-\frac{16 \pi^{2} \sigma_{r}^{2} \cos ^{2} \theta}{\lambda^{2}}\right](1.161),
$$

where $\theta$ is the angle of incidence of the illuminating beam. There results that the diffuse reflectance of such a rough surface is:

$$
R_{d}(\lambda)=R_{g}(\lambda)-R_{s p}(\lambda) \approx \frac{16 \pi^{2} \sigma_{r}^{2} \cos ^{2} \theta}{\lambda^{2}} R_{g}(\lambda)
$$

Usually, the percentage of the scattering in the light being reflected by the surface is specified by the "Total Integrated Scattering", or TIS of the surface, equal to:

$$
T I S=\frac{R_{d}}{R_{g}}=\frac{16 \pi^{2} \sigma_{r}^{2} \cos ^{2} \theta}{\lambda^{2}}
$$

\subsubsection{Typical transmittance and reflectance outputs}

It has been shown in section 1.4.1.4 that the spectral reflectance $R_{g}(\lambda)$ and transmittance $T_{g}(\lambda)$ of plane parallel samples can be obtained from the V-W configuration of conventional spectrophotometers This is illustrated in Figures 1.30a and 1.30b by the spectral transmittance graphs of several $2 \mathrm{~mm}$ thick samples. Figure 1.30 a concerns slabs of TAS $\left(\mathrm{Te}_{20} \mathrm{As}_{30} \mathrm{Se}_{50}\right)$ and $2 \mathrm{~S} 1 \mathrm{G}\left(\mathrm{Ge}_{15} \mathrm{Sb}_{20} \mathrm{~S}_{65}\right)$, and Figure $1.30 \mathrm{~b}$ a silicon plane parallel plate, polished on both sides. The curve on Figure $1.30 \mathrm{~b}$ shows the presence of the silicon cut-off wavelength at $1000 \mathrm{~nm}$, below which the light is completely absorbed before reaching the rear interface. In the transparency domain $(\lambda>1000 \mathrm{~nm})$, some light reaches the second interface and rebounds from it, so that the sample reflectance is very close to $\frac{2 R_{s}}{1+R_{s}}$,where $R_{s}$ is the reflectance at the interface air/sample. 

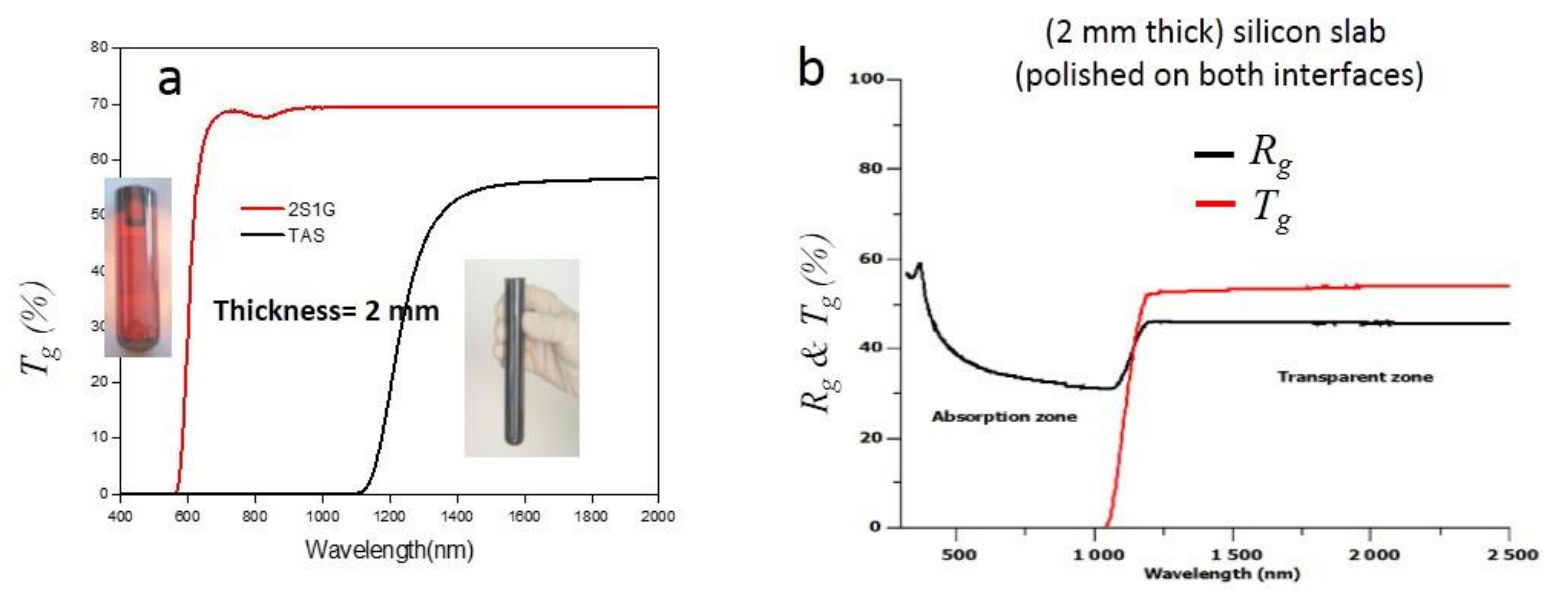

Figure 1. 30: a) Spectral transmittance of two infrared bulk materials (2S1G and TAS) measured with the $V$ configuration for normal incidence with air as reference. b) Spectral reflectance and transmittance of a thick silicon sample.

\subsubsection{Case of bulk materials}

\subsubsection{Sample with only one flat (polished) surface}

In this sub-section, it is supposed that the front surface of the sample is flat and well polished, and that the rear one is rough (diffuse). The V-W method does not have access to the transmittance of this kind of sample and consequently it can only deliver the spectral reflectance $R_{s}(\lambda)$ of its front surface, without any information on its transmission and absorption of the sample itself. However, if the Sellmeier formula of the constitutive material is known, it should be consulted to help check the experimental values of $R_{s}(\lambda)$, because this formula is a valuable tool for characterizing the dispersion of glasses and crystals. In fact, most optical glass manufacturers mention the Sellmeier coefficients of their materials in their catalogs.

If one does not know the material of the sample, the K-K relationships (equations 1.28, 1.29, and 1.30) may be used to deduce its refractive index and extinction coefficient from the experimental values of the spectral reflectance (section 1.1.2.1) obtained by the $W$ method. Since the reflectance measurements are realized inside a limited range $\left(\omega_{1}-\omega_{2}\right)$, in order to correctly apply the K-K relationships, one must analytically extrapolate the experimental reflectance values outside of this range, by means of an adjusted formula, such as the following one:

$$
\omega^{\prime}>\omega_{2} \quad R_{s}\left(\omega^{\prime}\right)=R_{s}\left(\omega_{2}\right)\left(\frac{\omega^{\prime}}{\omega_{2}}\right)^{a}
$$

where $a$ is an adjusted parameter $(0<a<4)$.

Alternatively, another extrapolation can be used [79]. Computational method to calculate $\varphi(\omega)$ must converge with high accuracy. Since it must take into account the singularity and to divide into a number of domains adapted to the $\omega$-broadband, for the integration calculation, the result of $\varphi(\omega)$ give directly the values of $n_{s}$ and $\kappa_{s}$ mentioned in section 1.1.2.1: 


$$
n_{s}=\frac{n_{0}\left(1-R_{s}(\omega)\right)}{1+R_{s}(\omega)-2 \cos (\varphi(\omega)) \sqrt{R_{s}(\omega)}} \text { and } \kappa_{s}=\frac{2 n_{0} \sin (\varphi(\omega)) \sqrt{R_{s}(\omega)}}{1+R_{s}(\omega)-2 \cos (\varphi(\omega)) \sqrt{R_{s}(\omega)}}
$$

The absolute uncertainties are the following:

$$
\begin{aligned}
& \sigma_{n_{s}}=\left|\frac{\left(1+R_{s}\right) \cos \varphi-2 \sqrt{R_{s}}}{\left(1+R_{s}-2 \sqrt{R_{s}} \cos \varphi\right)^{2} \sqrt{R_{s}}}\right| \sigma_{R_{s}}+\left|\frac{2 R_{s}\left(R_{s}-1\right) \sin \varphi}{\left(1+R_{s}-2 \sqrt{R_{s}} \cos \varphi\right)^{2} \sqrt{R_{s}}}\right| \sigma_{\varphi} \\
& \quad \text { and } \\
& \sigma_{\kappa_{s}}=\left|\frac{\left(R_{s}-1\right) \sin \varphi}{\left(1+R_{s}-2 \sqrt{R_{s}} \cos \varphi\right)^{2} \sqrt{R_{s}}}\right| \sigma_{R_{S}}+\left|\frac{2 R_{s}\left(\left(1+R_{s}\right) \cos \varphi-2 \sqrt{R_{s}}\right)}{\left(1+R_{s}-2 \sqrt{R_{s}} \cos \varphi\right)^{2} \sqrt{R_{s}}}\right| \sigma_{\varphi}(1.166)
\end{aligned}
$$

The accuracy of the K-K method is directly linked with the accuracy and value of $\varphi(\omega)$ for the extinction coefficient $\kappa_{s}$.

If we assume that the contribution of the light reflected from the (diffuse) second surface is negligible, it is found that the uncertainty upon the value of the refractive index is of the order of $\sigma_{n}=2 \%$ if $\sigma_{R s}=$ $1.10^{-3}$

\subsubsection{Plane parallel plate with two polished surfaces.}

In this case, the light having traversed the plate may be efficiently reflected back from the rear surface towards the front one, as shown in Figure 1.30b, and consequently, some information can be collected concerning the absorption and transparency spectral domains of the material.

The expressions 1.157 and 1.158 respectively become 1.167 and 1.168:

$$
\begin{gathered}
R_{g}=\frac{R_{s}\left[1+\left(T_{s}^{2}-R_{s}^{2}\right) \exp \left(-\alpha_{s} d_{s}\right)\right]}{1-R_{s}^{2} \exp \left(-\alpha_{s} d_{s}\right)}(1.167) \\
T_{g}=\frac{T_{s}^{2} \exp \left(-\frac{\alpha_{s} d_{s}}{2}\right)}{1-R_{s}^{2} \exp \left(-\alpha_{s} d_{s}\right)}(1.168)
\end{gathered}
$$

with

$$
\alpha_{s}=\frac{4 \pi}{\lambda} \kappa_{s}(1.169)
$$

where $d_{s}$ denotes the sample thickness.

In the transparency domain where $T_{g} \neq 0$, the refractive index $n_{s}$ is given by the analytic formulation below: 


$$
n_{s}=\frac{n_{0}\left(1+\sqrt{2 R_{g}-R_{g}^{2}}\right)}{R_{g}-1}
$$

The associated absolute uncertainty is:

$$
\sigma_{n_{s}}=\frac{n_{0}\left(1+\sqrt{2 R_{g}-R_{g}^{2}}\right)}{\left(1-R_{g}\right)^{2} \sqrt{2 R_{g}-R_{g}^{2}}} \sigma_{R_{g}}
$$

So it is found that $\sigma_{n_{s}}$ is around $0.5 \%$ and the absolute error $\sigma_{R_{g}}$ is of the order of $10^{-3}$.

In the totally absorbing zone, where $T_{\mathrm{g}}(\lambda)=0$, one can use the $\mathrm{K}-\mathrm{K}$ relation mentioned in section 1.5.1.1. For materials of low absorption such that $T_{\mathrm{g}}(\lambda)+R_{\mathrm{g}}(\lambda) \approx 1$ and for samples of known thickness $d_{s}$, analytical expressions cannot be obtained, and a computational method must be used in order to extract the values of the optical constants $\left(n_{s}\right.$ and $\left.\kappa_{s}\right)$.

It is important to note that the sample reflectance yields information on the optical band-gap $\left(E_{g}\right)$ of the material. The value of the corresponding cut-off wavelength $\lambda_{g},\left(\lambda_{g}(\right.$ in $\left.\mu \mathrm{m})=1.234 / E_{g}(\mathrm{in} \mathrm{eV})\right)$ is obtained by solving $\left(\frac{\partial^{2} R_{g}}{\partial \lambda^{2}}\right)_{\lambda=\lambda_{g}}=0$ [80].

We will see later how the optical constants $(n, \kappa)$ of a non-transparent substrate (or opaque metal layers) can be determined at normal incidence with the help of a transparent dielectric.

\subsubsection{Refractive index measurement of homogeneous dielectric thin films}

The determination of the optical parameters of weakly absorbing thin films is a problem often encountered in various sectors of applied physics, particularly in the micro-electronics and thin film technologies [81]. In order to optimize the design and fabrication of multilayers, it is essential to know precisely the refractive indices and extinction coefficients of the coatings. We will consider here the case of systems that are made up of a non-absorbing substrate coated with an absorbing thin film, as shown in the figure 1.31a and specified in the following way:

1) Its materials are isotropic and homogeneous, i.e. there is no variation of the refractive index throughout the film;

2) Both boundaries of the film are ideally flat, smooth and infinitely thin, i.e. without any transition layer;

3) The ambient medium is non-absorbing and of refractive index $n_{0}=1$

4) The (non-absorbing) substrate is a plane parallel plate; $R_{s}(\lambda)$ and $T_{s}(\lambda)$ are respectively the spectral reflectance and transmittance of each boundary; 
5) The global spectral quantities $T_{g}(\lambda)$ and $R_{g}(\lambda)$ measured in the $\mathrm{V}-\mathrm{W}$ configuration yield the theoretical reflectance $R(\lambda)$ and $R^{\prime}(\lambda)$ and the transmittance $T(\lambda)$ values of the coating defined by the equations 1.87 and 1.88 .

Figure $1.31 \mathrm{~b}$ shows the spectral transmittance $T_{\mathrm{g} 1}(\lambda)$ (equation 1.150) of the bare substrate and the spectral transmittance $T_{\mathrm{g} 2}(\lambda)$ of the coated substrate. $T_{\mathrm{g} 2}(\lambda)$ oscillates between two envelopes corresponding to its maximal $\left(T_{\max }\right)$ and minimal $\left(T_{\min }\right)$ values. In the same figure $1.31 \mathrm{~b}$ one notes the presence of four zones which are defined by the value of the ratio $z_{A}$ between the maximum transmittance of coated substrate and the transmittance of the bare substrate $\left(z_{A}=\frac{T_{\max }}{T_{g 1}}\right)$ [82]. These zones correspond to high $\left(z_{A}<0.4\right)$, moderate $\left(0.4<z_{A}<0.8\right)$, weak $\left(0.8<z_{A}<0.99\right)$ and null $\left(z_{A}=1\right)$ absorption of the sample (o). As far as reflectance is concerned, a similar behavior is observed, showing alternate maxima and minima. As previously described in sub-section 1.4.1.2, the oscillations observed between the two envelopes are interference fringes, and the spacing of these fringes is directly linked to the physical thickness of the film with respect to the wavelength, as is their number, which is also linked to the bandwidth of the covered domain.

We also note that the envelopes of $T_{\max }$ (or $R_{\min }$ ) respectively converge towards the transmittance (or reflectance) of the bare substrate, in the case of films of weak or no absorption at all. This characteristic is in general the signature of a homogeneous layer.
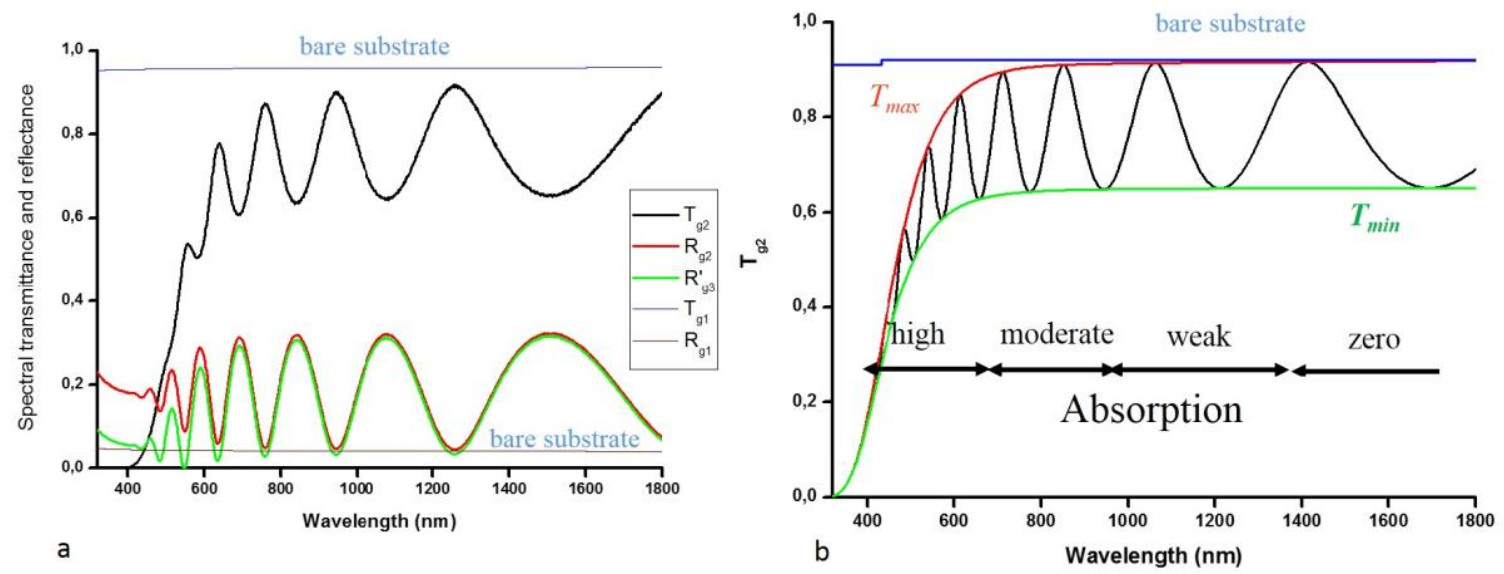

Figure 1.31: a) Spectral reflectance and transmittance of a bare and then coated substrate. b) Spectral transmittance $T_{\mathrm{g} 2}(\lambda)$ of a substrate with a homogeneous film, showing the envelopes of its maximum and minimum values. 
Over the spectral domain without any absorption, the theory [7] demonstrates that the locations of minima of transmittance (or maxima of reflectance) correspond to the value $Y_{\max }=\frac{n^{2}}{n_{s}}$ of the optical admittance with: $R_{\max }=\left|\frac{n_{0}-\frac{n^{2}}{n_{s}}}{n_{0}+\frac{n^{2}}{n_{s}}}\right|^{2} \quad$ (1. 172).

And the expression of the refractive index is then:

$$
n=\sqrt{\frac{n_{0} n_{s}\left(1+\sqrt{R_{\max }}\right)}{\left(1-\sqrt{R_{\max }}\right)}}
$$

In the weak absorption spectral domain of the film, the maximum value $T_{\max }$ of the transmittance and the minimum value $R_{\min }$ of the reflectance are affected. Manifacier et al. [82] demonstrated that the solution to the refractive index is expressed in terms of $T_{\max }$ and $T_{\min }$ :

$$
n=\sqrt{\varepsilon_{1}+\sqrt{\left(\varepsilon_{1}^{2}-\left(n_{0} n_{s}\right)^{2}\right)}} \text { (1. 174) with } \varepsilon_{1}=\frac{n_{0}^{2}+n_{s}^{2}}{2}+2 n_{0} n_{s} \frac{T_{\max }-T_{\min }}{T_{\max } T_{\min }} \text { (1. 175). }
$$

This method consists in detecting (Fig 1.31) the wavelengths for which reflectance is maximal and transmittance minimal. Ohlidal [83] suggests that the index of refraction is equal to:

$$
n=\sqrt{n_{s} \frac{\left(1+\sqrt{R_{\max }}\right)^{2}+n_{s} T_{\min }}{n_{s}\left(1-\sqrt{R_{\max }}\right)^{2}+T_{\min }}}
$$

Once the values of the refractive index are known at the wavelengths of two successive maximum $\left(T_{\max }\right)$ or minimum $\left(T_{\min }\right)$ values of the spectral transmittance, the film thickness $d$ is given by:

$$
d=\frac{\lambda_{1} \lambda_{2}}{2\left[n\left(\lambda_{2}\right) \lambda_{1}-n\left(\lambda_{1}\right) \lambda_{2}\right]}
$$

And the extinction coefficient of the film is given by:

$$
\kappa=\frac{\lambda}{4 \pi d} \ln \left(\frac{1-R}{T}\right)
$$

As far as uncertainties are concerned, if $\sigma_{n s}=\sigma_{R \max }=\sigma_{T \min }=1.10^{-3}$, and if $\sigma_{\lambda 1}=\sigma_{\lambda 2}=1 \mathrm{~nm}$, then the relative uncertainties are $\sigma_{n} / n=0.2 \%$ on the refractive index of the film and $\sigma_{d} / d=2 \%$ on its thickness. Since the optical constants $(n, k)$ are functions of wavelength, an iterative process involving smoothing steps of reflectance and transmittance and interpolation of the envelopes $R_{\max }$ and $T_{\min }$ is necessary to extract their values. These steps are important since the value of the wavelength corresponding to $R_{\max }$ (or $T_{\min }$ ) is the key for determining the thickness of the film. In fact, in order to extract the refractive index, the extinction coefficient and the thickness of the film from the measurements of the reflectance and transmittance of the system, this method, called the "R-T method", is an inverse problem. The analytical expressions of $n, \kappa$, and $d$ given by equations $(1.176,1.177$, and 1.178$)$ are being used as starting computational solutions to extract $R(\lambda)$ and $T(\lambda)$. 
The advantage of this method is that it is not based upon any approximation law for the refractive index (Cauchy, Sellmeier,...). However, it is necessary that the spectral reflectance graph shows at least one maximum in order to determine the film thickness. It must be noted that there is an infinite number of solutions for the refractive index values at the wavelengths corresponding to $T_{\max }$ and $R_{\min }$, hence a very large error bar in the vicinity of these wavelengths. In contrast, the resolved value of the refractive index is unique at the wavelengths corresponding to $T_{\min }$ and $R_{\max }$.

In order to increase the sensitivity of the results, choosing a high refractive index for the substrate can be useful when characterizing low index films. The terms: "high" and "low" indices are defined in relationship with the reflectance of the un-coated substrate: one refers to a film as being of high index if the reflectance of the film is higher than that of the substrate, and vice versa. Figure 1.32 shows the spectral curve of the refractive index of a Zinc sulfide (ZnS) thin film in the visible range, as obtained by means of the R-T method. The green curve corresponds to the average value of the refractive index. On either side of this curve, one finds the upper and lower limits $\left(+/-\sigma_{n}\right)$ of the uncertainty of the method, computed on the basis of a homogeneous layer with uncertainty values on reflectance and transmittance measurements equal to $\sigma_{R}=\sigma_{T}=1.10^{-3}$. However, we do observe that this uncertainty becomes much larger in the vicinity of wavelengths where the bare and coated substrates have similar reflectance.

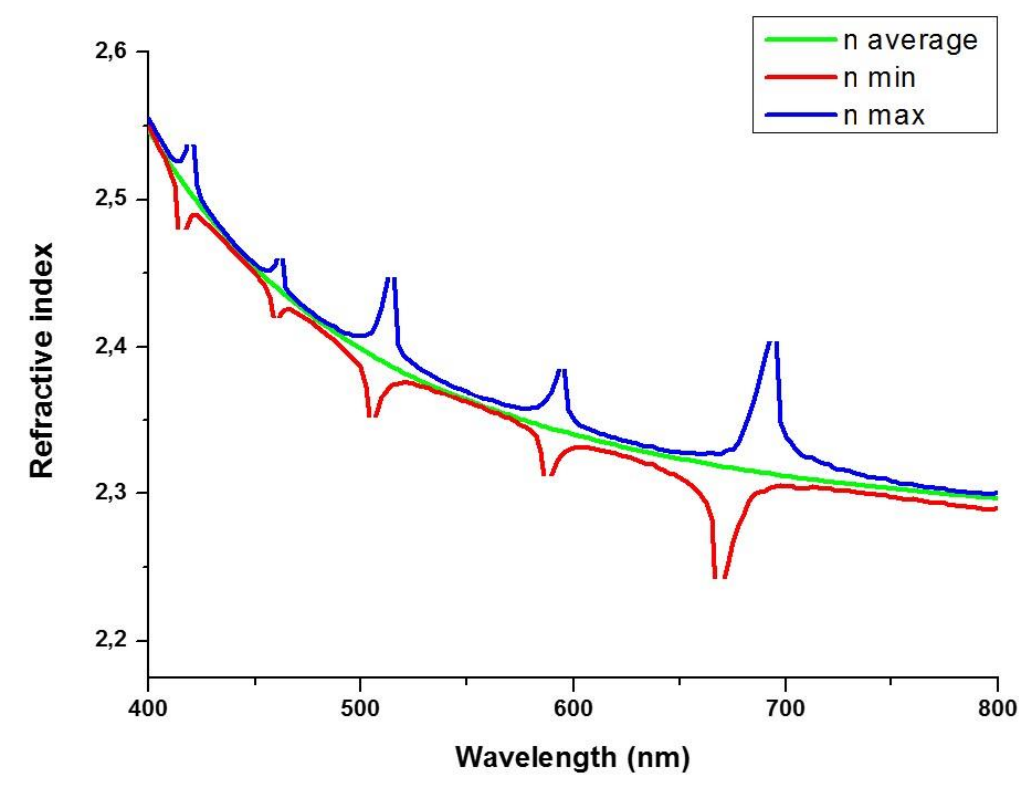

Figure 1.32: Typical result given by the $R$-T method about the refractive index of a ZnS thin film.

\subsubsection{Case of inhomogeneous dielectric thin films}

Depending upon the deposition process and conditions the spectrophotometric results may show that the maximal transmittance and minimal reflectance values of the coated substrate do not coincide with the transmittance or reflectance of the un-coated substrate (fig 1.33), whereas it is found that the energy conservation law: $R(\lambda)+T(\lambda)=1$, is verified. Spectrophotometry is utilized for the characterization of inhomogeneous films, particularly in the cases of columnar, or porous films, or of films with a graded composition. 


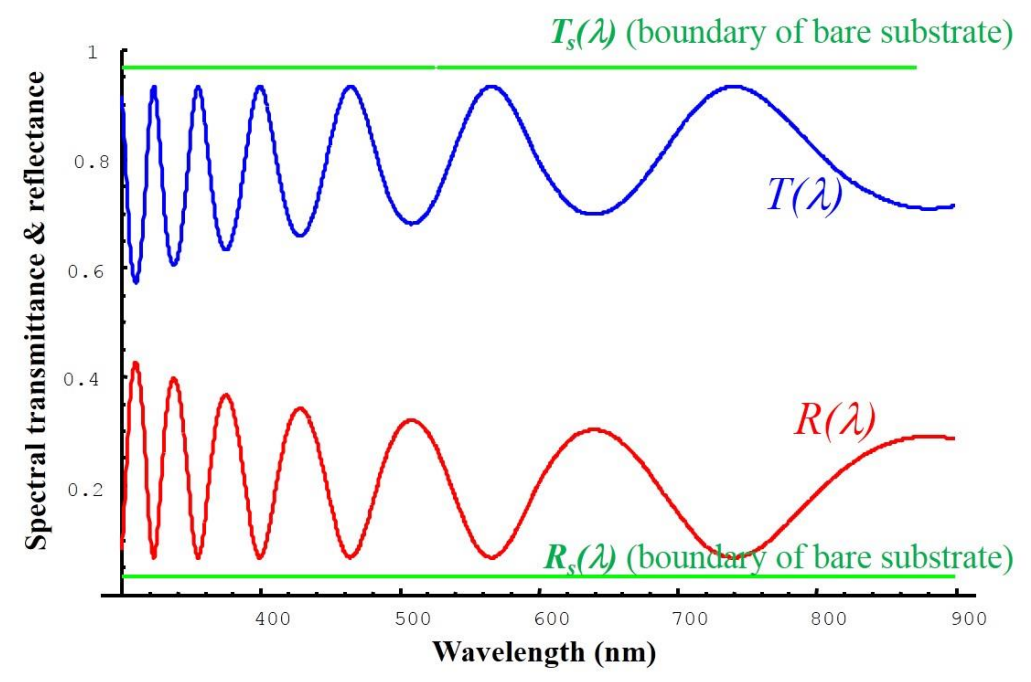

Figure 1.33: Spectral reflectance and transmittance of an inhomogeneous film deposited on a transparent substrate
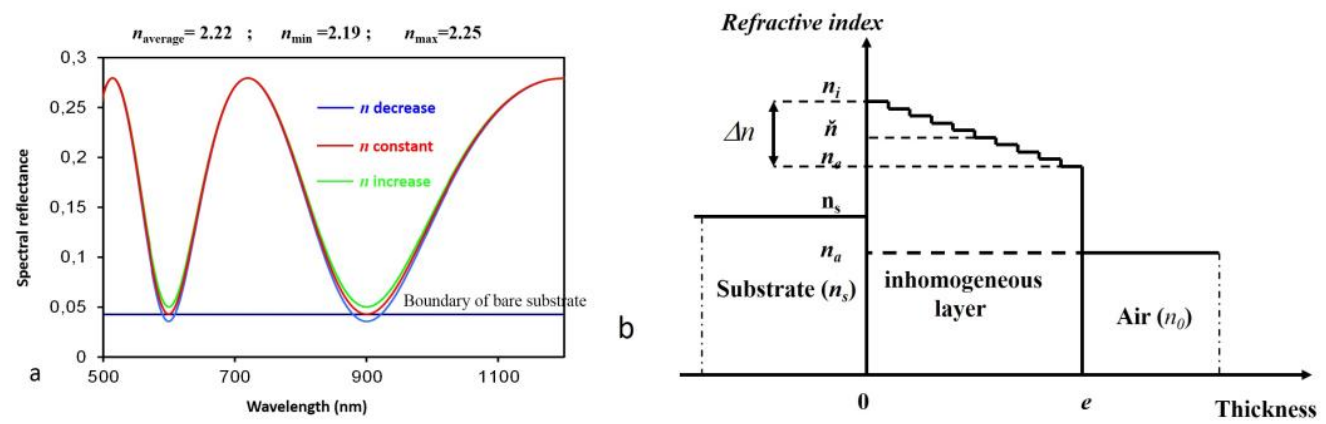

Figure 1.34:a) Comparison between the spectral reflectance of a transparent inhomogeneous layer with that of a layer with a linearly graded index (positive or negative gradient) b) Simulation of a linearly graded index by $N$ homogeneous layers for the case of a linear decreasing gradient (from substate to air).

In order to account for the inhomogeneity of a film, one may either consider the coating as being made up of $\mathrm{N}$ homogeneous sublayers with a linear index variation, or use the R. Jacobsson method [84], which is based upon_a matrix that takes into account the index variation with thickness. For normal incidence, the characteristic transfer matrix that links the electric field from one boundary to another is the following:

$$
M=\left[\begin{array}{cc}
\sqrt{\frac{n(\beta)}{n(0)}} \cos (\beta) & i \frac{\sin (\beta)}{\sqrt{n(\beta) n(0)}} \\
i \sqrt{n(\beta) n(0)} \sin (\beta) & \sqrt{\frac{n(0)}{n(\beta)}} \cos (\beta)
\end{array}\right]
$$

with $\beta=\frac{2 \pi}{\lambda} \int_{0}^{e} n(z) d z$ (1.180) 
The values of minimal reflectance are given by:

$$
R_{\text {min }}=\left(\frac{n_{0} n(\beta)-n(0) n_{s}}{n_{0} n(\beta)+n(0) n_{s}}\right)^{2}
$$

It must be noticed that the $R_{\max }$ values of the inhomogeneous film spectral reflectance differ from the $R_{s}(\lambda)$ values of the bare boundary. This criterion is to be used for differentiating inhomogeneous from homogeneous films.

The optical admittance $Y_{\max }$ at $R_{\max }$ is: $Y_{\max }=\frac{n(\beta) n(0)}{n_{s}}$

So the R-T method is well adapted to determining the refractive index of inhomogeneous films. With the starting solution to the relationship (1.176) established by Ohlidal [83], the numerical method explains optical properties of the film and extracts its optical constants and thickness. This method has been developed by J-P Borgogno and B. Lazaridès [85]. Other models exist such as that of linear gradient films which have been developed by Jacobsson [84] or by an approach of inverse synthesis of optical coating [86].

\subsubsection{Case of metallic films deposited on a transparent substrate}

This section deals with moderately or highly absorbing metallic films. In the case of a completely opaque layer $\left(T_{s}=0\right)$, since film admittance is equal to $n_{m}-i \kappa_{m}$, it is sufficient to determine $R_{s}(\lambda)$ and $R_{s}^{\prime}(\lambda)$ in order to compute the refractive index and extinction coefficient $\left(n_{m}\right.$ and $\left.\kappa_{m}\right)$ of the film. If the thin metallic film is not quite opaque, $R_{s}(\lambda), R_{s}{ }^{\prime}(\lambda)$ and $T_{s}(\lambda)$ are required. Analytical expressions of the optical properties $\left(T_{s}, R_{s}, R_{s}{ }^{\prime}, \frac{1+R_{s}}{T_{s}}, \frac{1-R_{s}}{T_{s}}, \frac{1+R_{s}{ }^{\prime}}{T_{s}}, \frac{1-R_{s}{ }^{\prime}}{T_{s}}(1.183)\right)$ can be found in papers of Heavens [74], F. Abeles [87] and S.G Tomlin [88].

These terms (1.183) are a priori sufficient to extract the optical constants $\left(n_{m}\right.$ and $\left.k_{m}\right)$ and the thickness of the metallic film $\left(d_{m}\right)$, assumed to be homogeneous. If a very thin metallic film is deposited, the search for its parameters can be based upon a power series of $\left(d_{m} / \lambda\right)$. If $d_{m} / \lambda<<1$ (1.184), a first order development [89], under normal incidence, allows to compute the product of the metallic layer parameters: $2 n_{\mathrm{m}} \kappa_{\mathrm{m}} d_{\mathrm{m}}$ in equations $1.185 \mathrm{a}, 1.185 \mathrm{~b}$ and $1.185 \mathrm{c}$ depending of experimental measurements:

$$
\begin{aligned}
& 2 n_{m} \kappa_{m} d_{m}=\frac{\lambda}{2 \pi} \frac{n_{s}(\lambda)}{n_{s}(\lambda)-n_{0}(\lambda)} \frac{R_{s}(\lambda)-R_{s}{ }^{\prime}(\lambda)}{T_{s}(\lambda)} \text { (1.185a) } \\
& 2 n_{m} \kappa_{m} d_{m}=\frac{\lambda}{2 \pi} n_{s}(\lambda) \frac{1-R_{s}(\lambda)-T_{s}(\lambda)}{T_{s}(\lambda)}=\frac{\lambda}{2 \pi} n_{s}(\lambda) \frac{A_{s}(\lambda)}{T_{s}(\lambda)} \text { (1.185b) } \\
& 2 n_{m} \kappa_{m} d_{m}=\frac{\lambda}{2 \pi} n_{0}(\lambda) \frac{1-R_{s}{ }^{\prime}(\lambda)-T_{s}(\lambda)}{T_{s}(\lambda)} \text { (1.185c) }
\end{aligned}
$$

where $R_{s}{ }^{\prime}\left(A_{s}^{\prime}\right)$ and $R_{\mathrm{s}}\left(A_{s}\right)$ are the film reflectance (absorptance) respectively on the substrate and air sides, and $T_{\mathrm{s}}$ is its transmittance. 
This development shows how it is difficult to correctly evaluate the optical constants of a very thin metallic layer. Although the method described above delivers a partial solution, which is the product of $n_{m}, \kappa_{m}$, and $d_{m}$, it remains inadequate.

\subsubsection{Optical constant determination by the bilayer « metallic- dielectric » method}

Since metallic layers can be inhomogeneous, depending upon their deposition process and conditions, there is a need for a more efficient method dedicated to determine their optical properties. That is the goal of the bilayer "metallic -dielectric" method, exposed below in two modes: the "Rs-Rc method" for opaque metallic films, and the "RsTsRcTc method" for non opaque ones.

\subsubsection{The "Rs-Rc method" for opaque metallic films}

Let the thick and opaque metallic layer to be characterized, of optical constants $n_{m}$ and $k_{m}$, be deposited upon a transparent substrate, be considered as a substrate. The spectral reflectance of its front surface $R_{s}(\lambda)$ is measured, and the spectral transmittance of its front and rear surfaces, $T_{s}(\lambda)$, is set equal to 0 . Then, a dielectric film is deposited on top of the metallic film, and the spectral reflectance $R_{c}(\lambda)$ of the coated metallic film is measured under the hypothesis that there is no physico-chemical interaction at the interface metal-dielectric. Figure 1.35a shows the two phases of the $R_{s}-R_{c}$ method, and Figure $1.35 \mathrm{~b}$ the typical corresponding spectral reflectance graphs.
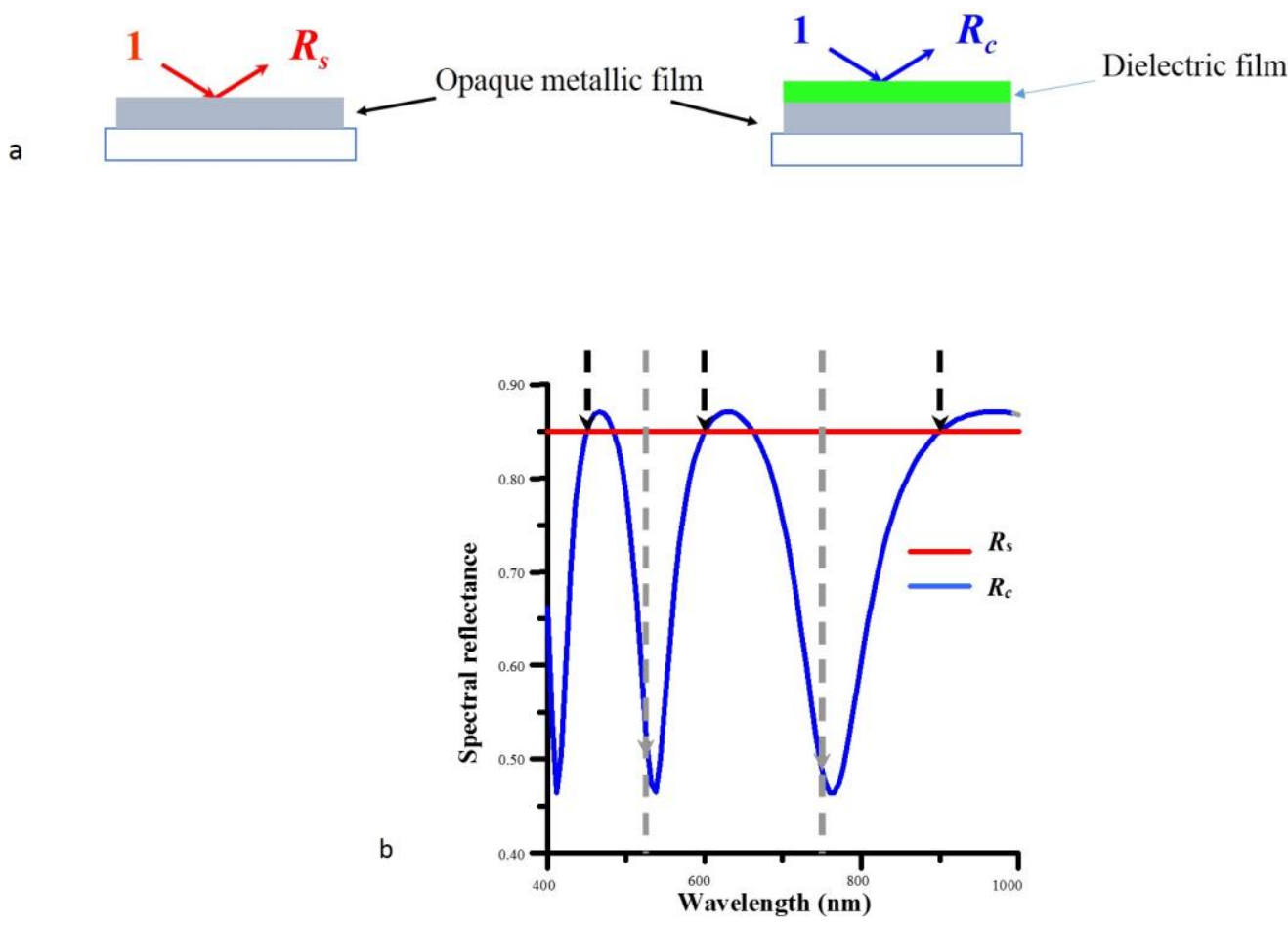

Figure 1.35: a) The two configurations of the $R_{s} \cdot R_{c}$ method b) corresponding spectral reflectance $R_{s}(\lambda)$ of metallic film alone, and $R_{c}(\lambda)$ of the same metallic film coated with a dielectric film.

At each wavelength, one can write the following set of equations: 


$$
\begin{gathered}
\left(n_{m}-n_{0} \frac{1+R_{s}}{1-R_{s}}\right)^{2}+\kappa_{m}^{2}=4 n_{0}^{2} \frac{R_{s}}{\left(1-R_{s}\right)^{2}} \text { (1. 186) } \\
\kappa_{m}=\frac{n_{d}^{2}+n_{0}^{2}}{2 n_{d}} \operatorname{tg} \varphi_{d}+\frac{2 n_{0}}{n_{d}\left(n_{d}^{2}-n_{0}^{2}\right)} \frac{1}{\sin 2 \varphi_{d}}\left\{\frac{1+R_{s}}{1-R_{s}}\left(n_{d}^{2} \cos ^{2} \varphi_{d}+n_{0}^{2} \sin ^{2} \varphi_{d}\right)-n_{d}^{2} \frac{1+R_{c}}{1-R_{c}}\right\} n_{m}
\end{gathered}
$$

with $\varphi_{d}=\frac{2 \pi}{\lambda} n_{d} d_{d}$ the phase shift introduced by the dielectric film of thickness $d_{\mathrm{d}}$.

One will notice that, in the $n_{\mathrm{m}}, \kappa_{\mathrm{m}}$ plane, equation (1.186) represents a circle centered the point of coordinates $n_{m}=n_{0} \frac{1+R_{s}}{1-R_{s}}$ and $\kappa_{m}=0$, having a radius equal to $\quad 2 n_{0} \frac{\sqrt{R_{s}}}{1-R_{s}}$; equation 1.187 is that of a straight line $\left(\kappa_{m}=\kappa_{m_{0}}+A_{\kappa} n_{m}\right)$ representative of a linear dependence of $\kappa_{\mathrm{m}}$ with respect to $n_{m}$ of slope $A_{\kappa}$ The solution to the problem of index determination is given by the coordinates of the intersection points between the circle and the straight line. As shown by Figure 1.36, there may be several solutions. One way to eliminate this ambiguity is to make measurements on two systems coated with layers of the same metal but of different dielectric thicknesses, then the solution is unique.

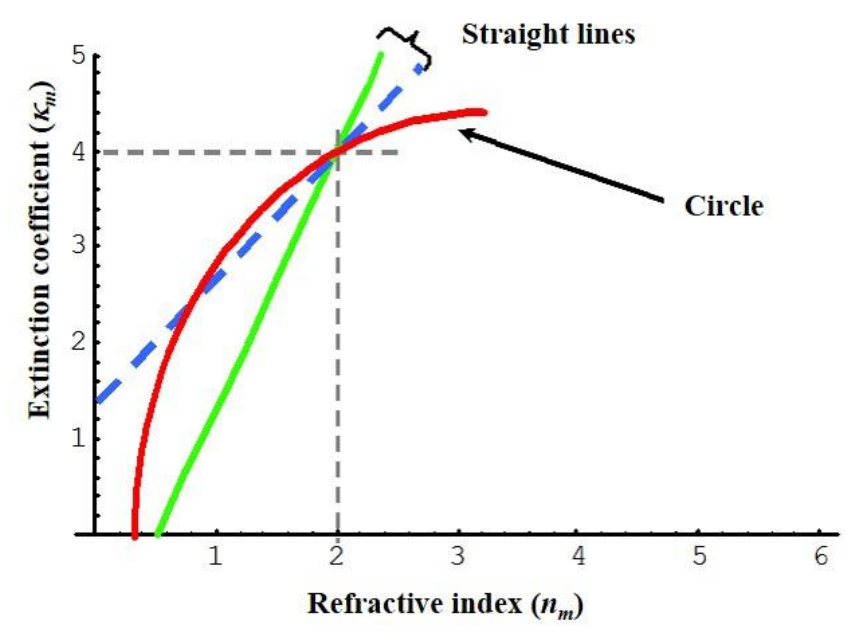

Figure 1.36: Graphical representation of the search for solutions of the optical constants of a metallic substrate or opaque metal layer $\left(n_{m}=2, \kappa_{m}=4\right)$ and $n_{d}=2.35$ for two phase retardations $\varphi_{d}=\pi / 4$ and $\varphi_{d}$ $=3 \pi / 4$.

The measurements should be done for a large number of wavelengths over the spectral range of interest.

It can been shown, at the wavelengths where $R_{s}=R_{c}$, that $\varphi_{d}=p \pi$ ( $p$ integer) for the wavelengths of intersection where the slope of the $R_{c}$ curve is positive (figure 1.35b). For these wavelengths, the equation 1.187 shows that the extinction coefficient $\kappa_{m} \rightarrow \infty$.

If the wavelength values for which $\varphi_{d}=(p+1 / 2) \pi$, the formulae 1.187 allows to determine the refractive index $n_{m}$ and the extinction coefficient $\kappa_{m}$ by: 


$$
n_{m}=\frac{\left(n_{d}^{4}-n_{0}^{4}\right)}{2 n_{0}\left(n_{d}^{2} \frac{1+R_{c}}{1-R_{c}}-n_{0}^{2} \frac{1+R_{s}}{1-R_{s}}\right)}(1.188 \mathrm{a}) \quad \text { and } \quad \kappa_{m}^{2}=\frac{\left(n_{0}-n_{m}\right)^{2}-R_{s}\left(n_{0}+n_{m}\right)^{2}}{\left(R_{s}-1\right)}
$$

These expressions of refractive index and extinction coefficient can be considered as the starting solutions for iterative calculations.

Considering realistic values of uncertainties upon the reflectance, the reflective index and the thickness of the dielectric layer (such as: $\sigma_{R_{s}}=\sigma_{R_{c}}=0.1 \%, \sigma_{n_{d}}=0.2 \%$ and $\sigma_{d}=1.5 \mathrm{~nm}$ ), the resulting $r m s$ uncertainties on $n_{m}$ and $\kappa_{m}$ are similar, between $1 \%$ and $2 \%$.

If this method is less accurate than the one based upon the K-K relationships, it has the following advantages:

- The investigated spectral range can be limited at will

- No analytical extrapolation, as is the case for K-K relationships

- No use of approximate laws for the optical constants, as is the case in R-T method

- In situ reflectance measurements $\left(R_{s}\right.$ and $\left.R_{c}\right)$ can be performed

- Method applicable to materials with strong to moderate absorption zones

The disadvantages of this method are:

- The dielectric film must be homogeneous

- The dielectric layer parameters $\left(n_{d}\right.$ and $\left.d\right)$ must be precisely characterized

- Two reflectance values must be measured

- The method accuracy is low for weakly absorbing layers

- The method is critical if one needs to know the wavelength values accurately

- Improving the accuracy necessitates the use of high index dielectrics

This " $R_{s} R_{c}$ method" has been successfully used to measure the refractive indices of materials such as aluminum, chromium, hafnium, and nickel [92]. Figure 1.33 shows the experimental characterization of these opaque metallic films [92] with this method.

For each of these metals, a high refractive index $(>2)$ dielectric layer was associated with the metallic film. In the case of aluminum, nickel and chromium, the films were deposited via the electron beam deposition (EBD) technology and a BAK 600 coating system, and then covered by a ZnS dielectric coating. In the case of Hafnium, the dielectric coating was in Hafnium dioxide $\left(\mathrm{HfO}_{2}\right)$, both films being deposited with the Reactive Low Voltage Ion Plating (RLVIP) technology and a BAK 800 coating system. The optical measurements were carried out using the $\mathrm{V}-\mathrm{W}$ configuration (incidence angle of $8^{\circ}$ ) of a Perkin-Elmer spectrophotometer model LAMBDA-19. Figure 1.38 shows the spectral curves for each one of these bilayers, i.e. $R_{s}(\lambda)$ (metallic layer alone) and $R_{c}(\lambda)$ (metal + dielectric layers), along with the optical constants $\left(n_{m}\right.$ and $\kappa_{m}$ ) of the opaque metallic films resulting from the use of the $R_{s} R_{c}$ method. These results are in agreement with the ones obtained on bulk materials [Hass [94], Palik[21]]. 

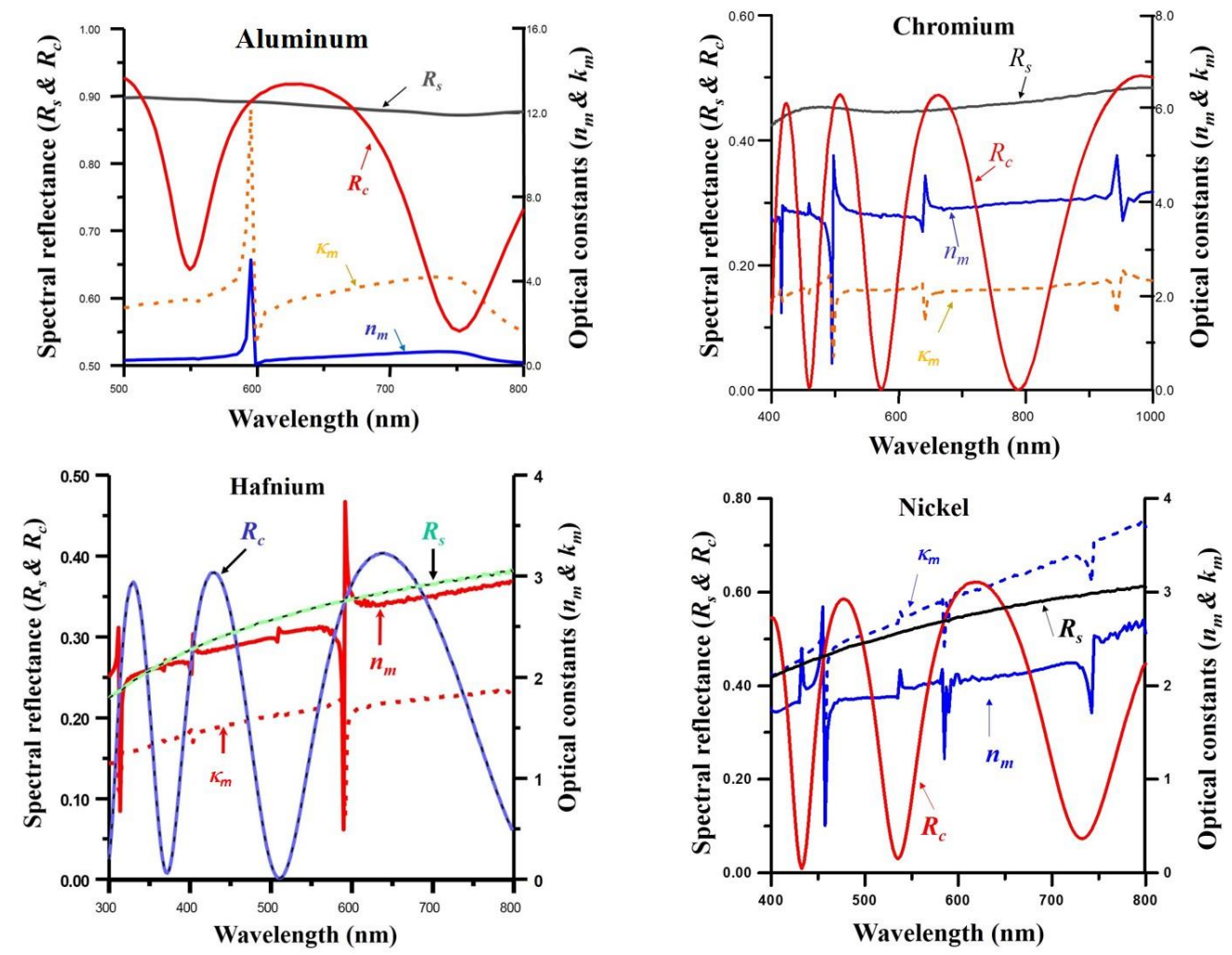

Figure 1.37: Experimental characterization of opaque metallic films by the $R_{s}-R_{c}$ method: Aluminum, Chromium, Hafnium, Nickel

\subsubsection{Case of metal-dielectric bilayer with non-opaque metallic layers}

In this case, the metallic film may be traversed by some fraction of the illuminating beam, and its thickness $d_{m}$ plays an important role.

In constrast to the $R_{s}-R_{c}$ method, as described by figure $1.38,4$ spectral measurements $\left(R_{s}(\lambda), T_{s}(\lambda)\right.$, $\left.R_{c}(\lambda), T_{c}(\lambda)\right)$ are necessary to obtain the 3 unknown parameters (i.e. $n_{m}, \kappa_{m}$, and $d_{m}$ ). Figure 1.39 shows the corresponding schematic diagram.

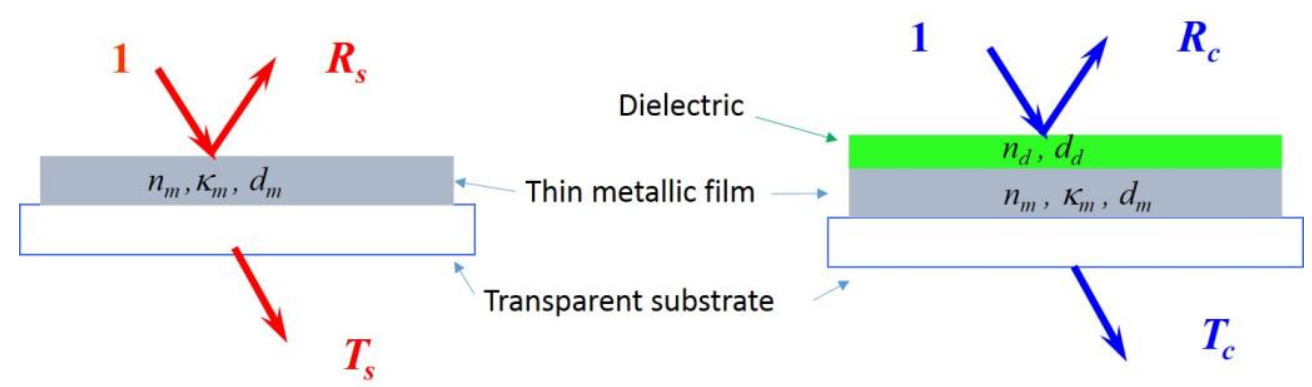


Figure 1.38: Schematic diagram showing the parameters and measured quantities of a bilayer system: a) transparent substrate coated with a non totally opaque metallic film (b) same system covered with an additional dielectric layer.

Electromagnetic theory shows that the potential transmittance, $\psi^{T}$, of any element of a coating system is defined as the ratio of the output to the input irradiances, the input being the net irradiance rather than the incident: $\psi^{T}=\frac{T}{1-R}$. So:

a) Potential transmittance is equal to $100 \%$ only if all constituents (coatings and substrate) are non absorbing (which is the case of dielectric film).

b) If the system is made up of transparent substrate with of non opaque metallic layer: $\psi_{s}^{T}=\frac{T_{s}}{1-R_{s}}$ where $T_{s}$ and $R_{s}$ are respectively the transmittance and the reflectance of the system (fig1.38a).

c) After deposition of the non absorbing dielectric, for the bilayer (metal+dielectric) the potential transmittance is given by $\psi_{c}^{T}=\frac{T_{c}}{1-R_{c}}=\psi_{s}^{T}$ (figure 1.38b)

Now, if one considers the case of a thin metallic film, $\frac{d_{m}}{\lambda}<<1$ the Wolter relationships (equation 1.185) give important information on the optical constants and thickness of the metallic film.

$$
2 n_{m} \kappa_{m} d_{m}=\frac{\lambda}{2 \pi} n_{s} \frac{A_{s}}{T_{s}}=\frac{\lambda}{2 \pi} n_{s} \frac{A_{c}}{T_{c}}
$$

where $A_{s}$ and $A_{c}$ are respectively the absorptances of the following coatings: metal alone and metal + dielectric. The most important information from relation 1.190 is that, if $\psi_{s}^{T} \neq \psi_{c}^{T}$, one may consider that there exists some "chemical interaction" between metallic and dielectric film, at interface and/or bulk film.

Now, if it is assumed that there is no such interaction, on may consider that optical admittance of thin metallic film is , $Y_{m}=X_{m}-i Z_{m}$, where $X_{m}$ and $Z_{m}$ are functions of the optical constants and thickness of the metallic film. In that case $X_{m}$ stands for $n_{m}$ and $Z_{m}$ stands for $\kappa_{m}$ in equations 1.186 and 1.187 .

In the case of very thin metallic films, it is more difficult to dissociate the three parameters $n_{m}$, $\kappa_{m}$ and $d_{m}$. This can be done by numerical calculations [95] with a complex strategy using different merit functions of $T_{s}, R_{s}, R_{c}$, and $T_{c}$ [91-92], where:

- $R_{s}$ and $T_{s}$ are the air-side reflectance and transmittance of the single metallic film;

- $R_{c}$ and $T_{c}$ are the air-side reflectance and transmittance after deposition of the dielectric layer on metallic film.

For information, the strategy to determine optical constants is based on the use of the expressions of $(1-R) / T$ and $(1+R) / T$ [7-88-90-92].

This strategy has been used for nickel films of different thicknesses, obtained by an electron beam deposition technique: the results delivered by the numerical algorithm for these three parameters are satisfactory for all thicknesses. 
However, a notable discrepancy is observed for each parameter $\left(n_{m}, \kappa_{m}\right)$ if one compares the results obtained for different thicknesses, which means that metallic films are inhomogeneous [9192].

A simulation (figure 1.39) based upon the change in compactness inside a metallic layer [91], including the Maxwell-Garnett model, has been successfully developed to fabricate a broadband absorber (absorptance $A>99 \%$ ) which operates over the visible [91-94].

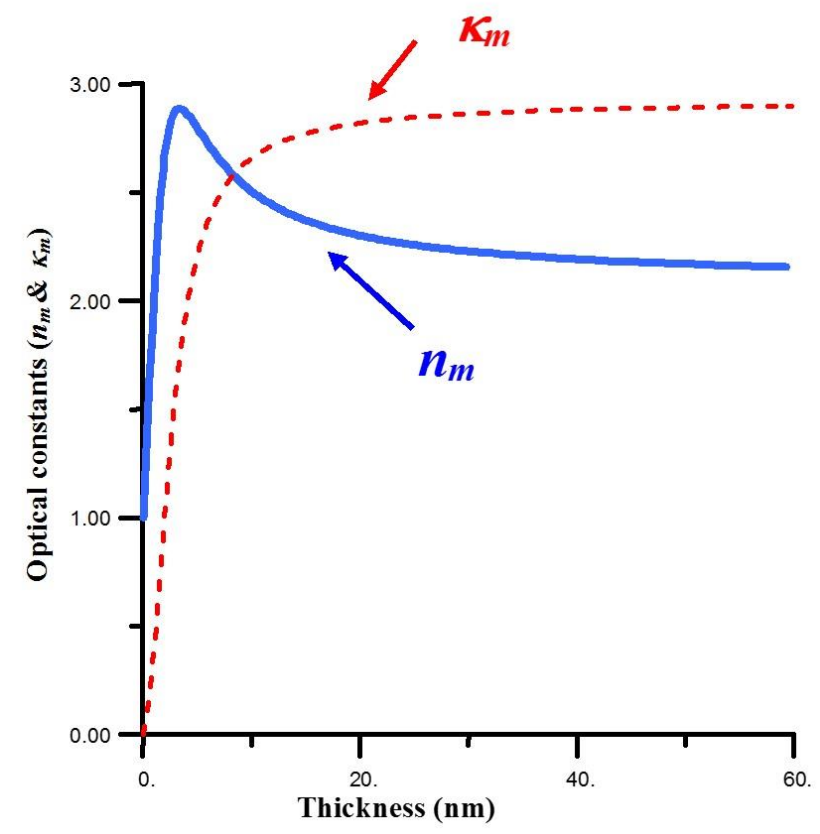

Figure 1.39: Simulation of optical constants, versus thickness of a Nickel layer at $\lambda=600 \mathrm{~nm}$ [91]

In conclusion of this section, measuring the refractive index is a more difficult task for a non-opaque metallic film than for a dielectric film. Furthermore, it is important to note that the layer model must be chosen in connection with its deposition technology, and that the best results are obtained with homogeneous layers.

Spectrophotometry is a simple tool to use, well adapted to the ex situ and in situ characterization of thin films and for the determination of optical constants. It provides a guidance for choosing the appropriate layer model. Every three years, at its Optical Interference Coatings conference, international optical coating community launches a measurement challenge [96-99]. 


\section{Bibliography}

[1] www.schott.com

[2] N. G. Van Kampen, F. Lurçat, "Causalité et relations de Kramers-Kronig," Le journal de Physique et le Radium, 22, 179-191 (1961). Trad. Nederlands Tidschrift voor Natuurkunde, 24, 1-14 en 29-42 (1958).

[3] V. Lucarini, J. J. Saarinen, K.-E.Peiponen, M. E. Vartiainen, Kramers-Kronig relations in optical materials (Springer, Verlag, Berlin Heidelberg 2005).

[4] J. F. Ogilvie, G. J. Fee, "Equivalence of Kramers-Kronig and Fourier transforms to convert between optical dispersion and optical spectra," MATCH Comm. Math. Comput. Chem. 69, 249-262 (2013).

[5] M. Born, E. Wolf, Principles of optics: electromagnetic theory of propagation, interference and diffraction of light (Cambridge University Press, 1999).

[6] A. Hadni, Essentials of modern physics applied to the study of the infrared (Pergamon Press, Oxford 1967)

[7] H. A Macleod, Thin-film optical filters Fourth Edition (CRC Press, Taylor\&Francis Group, Boca Raton London New-York, 2010)

[8] F. Sh, Forman, AV Tikhonravov, Basics of optics of multilayer systems (Gif-sur-Yvette, Edition Frontieres, 1992)

[9] PTB Website, https://www.ptb.de/cms/en.html (2015)

[10] D.Tentori, J.R. Lerma: "Refractometry by minimum deviation accuracy analysis", Opt. Eng. 29(2), $160-168$ (1990)

[11] D.B. Leviton, B.J. Frey, T.K. Kvamme: "High accuracy, absolute, cryogenic refractive index measurements of infrared lens materials for JWST NIRcam using CHARMS", Proc. SPIE 5904 (2005)

[12] B.J. Frey, D.B. Leviton: "CHARMS: the cryogenic, High accuracy refraction measuring system NIST High accuracy refractometry-UV/vis", http://www.nist.gov/ (2015)

[13] C. Véret: Réfractométrie, Article R 6300 Techniques de l'ingénieur, Volume " Mesures et contrôle » (1995).

[14] J. Mangin, "Indice de réfraction des matériaux optiques massifs", CNRS/ROP Workshop on metrology of refractive indices, Paris, 24-25/11/2008 The corresponding original lecture has been integrally reproduced one year later by one of the present co-authors (J.-L. Meyzonnette) at URL http://www.rop.cnrs.fr/IMG/pdf/Indices de refraction.pdf.(2009).

[15] R.M.A. Azzam, N.M. Bashara: Ellipsometry and polarized light. (Elsevier, Amsterdam, 1987) 
[16] G. Tompkins, and W. A. McGahan, Spectroscopic Ellipsometry and Reflectometry: A User's Guide, (Wiley, New York, 1999).

[17] H. G. Tompkins and E. A. Irene (eds.), Handbook of Ellipsometry, (William Andrew, Norwich, New York, 2005).

[18] A. Rothen, "The ellipsometer, an apparatus to measure thicknesses of thin surface films", Rev. Sci. Instrum., 16, 26-30 (1945) htpp://doi.org/10.1063/1.1770315

[19] F. Abeles, "Surface electromagnetic waves ellipsometry", Surface Science 56: 237-251 (1976)

[20] D. E. Aspnes, J. B. Theeten, and F. Hottier. "Investigation of effective-medium models of microscopic surface roughness by spectroscopic ellipsometry." Physical Review B 20.83292 (1979)

[21] E.D Palik, Handbook of Optical Constants of Solids, (Academic Press, New-York, 1985)

[22] J. A. Faucher, George M. McManus, and Hans J. Trurnit: "Simplified Treatment of Ellipsometry" JOSA Vol. 48, Issue 1, pp. 51-54 (1958)

[23] S. Huard, Polarisation de la lumière (Ed Masson 1994)

[24] R.C Jones, "A new calculus for the treatment of optical systems Part I", JOSA, 31, 486-493 (1941)

[25] R.C Jones, "A new calculus for the treatment of optical systems Part III", JOSA, 31, 500-503 (1941)

[26] R.C Jones, "A new calculus for the treatment of optical systems Part IV", JOSA, 32, 488-493 (1942)

[27] D.E. Aspnes, "Spectroscopic ellipsometry - Past, present, and future", Thin Solid Films 571 (2014) 334-344

[28] JM Frigerio:"Détermination des indices par ellipsométrie : Principes théoriques et limitations" Workshop MRCT-CNRS http://www.rop.cnrs.fr/spip.php?article349 (2008 and 2009)

[29] F. Bernoux, J. P. Piel, B. Castellon, C. Defranoux, J. H. Lecat, P. Boher, \& J. L. Stehle, EllipsométrieThéorie. Traité Mesures et Contrôles, Techniques de l'Ingénieur, 1-16. (2003)

[30] L. Prod'homme, "A new approach to the thermal change in the refractive index with temperature," Phys. Chem. Glasses 1, 145-153 (1960).

[31] A. J. Bosman, E. E Havinga, "Temperature dependence of dielectric constants of cubic ionic compounds," Phys. Rev. 129, 1593-1600 (1963).

[32].E. E. Havinga, A. J. Bosman, "Temperature dependence of dielectric constant of crystals with $\mathrm{NaCl}$ and CsCl structure," Phys. Rev. 140, A292-A303 (1965).

[33] J. M. Jewell, "Model for the thermo-optic behavior of sodium borate and aluminosilicate," J. NonCryst. Solids,. 146, 145-153 (1992). 
[34] G. Gosh, "Sellmeier coefficients and dispersion of thermo-optic coefficients for some optical glasses," Appl. Opt. 36, 1540-1546 (1997).

[35] Zhang Ting, Wu Meng-Qiang, Zhang Shu-Ren, Xiong Jie, Wang Jin-Ming, Zhang Da-Hai, He FengMei, Li Zhong-Ping, "Permittivity and its temperature dependence in hexagonal structure $B N$ dominated by the local electric field," Chin. Phys. B 21, 077701-1_077701-8 (2012).

[36] G. N. Ramachandran, "Thermo-optic of solids," Proc. Indian Acad. Sci., 25A, 498-515 (1947); Ibidem, 25A, 280-286 (1947).

[37] J. E. Rapp, H. D. Merchant, "Thermal expansion of alkali halides from 70 to 570 K," J. Appl. Phys. 44, 3919-3923 (1973).

[38 ] S. Kumar, "Thermal expansion of simple ionic crystals," Proc. Nat. Inst. Sc. India A25 44, 364-372 (1959).

[39] D. B. Sirdeshmukh, L. Sirdeshmukh, K. G. Subhadra, Alkali halides: a handbok of physical properties, (Springer-Verlag, Berlin Heidelberg, 2001).

[40] H. H. Li, "Refractive index of alkali halides and its temperature derivatives," J. Phys. Chem. Ref. Data 5, 329-528 (1976).

[41] M. Lallemand, J. Martinet, "Influence de la température sur le coefficient thermo-optique des fluorures alcalino-terreux," Rev. Phys. Appl. 17, 111-117 (1982).

[42] K. F. Trost, "Die thermische Ausdehnung der Alkalihalogenide vom NaCl-Typ bei hohen und tiefen Temperaturen," Z. Naturforschg. 18b, 662-664 (1963).

[43] https://www.corning.com/

[44] http://www.korth.de/

[45] www.ohara-inc.co.jp

[46] www.schott.com/advanced optics

[47] www.vitron.de/english/

[48] http://www.hoyaoptics.com

[49] www.hikari-g.co.jp

[50] www.opticalmaterials.umicore.com

[51] www.amorphousmaterials.com

[52] https://www.fiberlabs-inc.com/

[53] D. B. Leviton, B. J. Frey, "Temperature dependent absolute refractive index measurements of fused silica," Proc.SPIE 6273, 6273K (11p.) (2006). 
[54] B. D. Frey, D. B. Leviton, "Automation, operation and data analysis in the cryogenic, high accuracy, refraction measuring system (CHARMS)," Proc. SPIE 5904,212-221 (2005).

[55] J. F. Nye, Physical properties of crystals (Oxford, Clarendon Press, 1976).

[56] S. Fossier, S. Salaün, J. Mangin, O. Bidault, I. Thénot, J.-J. Zondy, W. Chen, F. Rotermund, V. Petrov, J. Heningsen, A. Yelisseiev, L. Isaenko, S. Lobanov, O. Balachninaite, G. Slekys, V. Sirutkaitis, "Optical, vibrational, thermal, electrical, damage and phase-matching properties of lithium thioindate," J. Opt. Soc. Am. B, 21, 1981-2007 (2004).

[57] J. Mangin, G. Mennerat, G. Gadret, V. Badikov, J.-C. de Miscault, "Comprehensive formulation of the temperature dependence dispersion of optical materials; illustration with case of temperature tuning of a mid-IR $\mathrm{HgGa}_{2} \mathrm{~S}_{4}$ OPO," J. Opt. Soc. Am. B, 26, 1702-1709 (2009).

[58] J. Mangin, P. Strimer, L. Lahlou-Kassi, "An interferometric dilatometer for the determination of thermo-optic coefficients of NLO materials," Meas. Sci. Technol. 4, 826-834 (1993).

[59] G. E. Merritt, "The interference method of measuring thermal expansion," J. Res. Nat. Bur. Stand. (U. S.), 10, 59-76 (1932).

[60] R. M. Walker, G. W. Cleek, I. H. Malitson, M. J. Dodge, T. A. Hahn, "Optical and mechanical properties of some neodymium-doped glasses," J. Res. Nat. Bur. Stand. (U. S.), 75A, 163-174 (1971).

[61] R. M. Walker, G. W. Cleek, "Refractive index of fused silica at low temperatures," J. Res. Nat. Bur. Stand. (U. S.), 75A, 279-281 (1971).

[62] R. M. Walker, G. W. Cleek, "The effect of temperature and pressure on the refractive index of some oxide glasses," J. Res. Nat. Bur. Stand. (U. S.), 77A, 755-763 (1973).

[63] M. Okaji, H. Imai, "A practical measurement system for accurate determination of linear thermal expansion coefficients," J. Phys. E: Sci. Instrum. 17, 669-673 (1984).

[64] P. Hariharan, D. Sen, "Double-passed two-beam interferometers -II- Effect of specimen absorption and finite path difference," J. Opt. Soc. Am. 51, 1212-1218 (1961).

[65] A. P. Müller, A. Cezairlaiyan, "Interferometric technique for the subsecond measurement of thermal expansion at high temperatures: application to refractory metals," Int. J. of Thermophys. 12,643-656 (1991).

[66] G. Gosh, "Model for the thermo-optic coefficients of some standard optical glasses," J. Non-Cryst. Solids 189, 191-196 (1995).

[67] W.J. Tropf, M. E. Thomas, T. J. Harris, "Optical and physical properties of crystals and glasses," in Handbook of optics vol II, part 4 ch. 33, (Mc-Graw-Hill Inc. 1995).

[68] M. V. Hobden, J. Warner, "The temperature dependence of the refractive indices of pure lithium niobate", Phys. Lett., 22, 243-244 (1966). 
[69] D. H. Jundt, "Temperature-dependent Sellmeier equation for the index of refraction, $n_{e}$, in congruent lithium niobate," Opt. Lett. 22, 1553-1555 (1997).

[70] I. Dolev A. Ganany-Padowicz O. Gayer A. Arie-J. Mangin G. Gadret, "Linear and nonlinear optical properties of $\mathrm{MgO}: \mathrm{LiTaO}_{3}$," Appl Phys B 96, 423-432, (2009).

[71] J. Mangin, G Mennerat, P Villeval, "Thermal expansion, normalized thermo-optic-coefficients, and condition for second harmonic generation of a Nd:YAG laser with wide temperature bandwidth in $\mathrm{RbTiOPO}_{4}, "$ J. Opt. Soc. Am. B, 28, 873-881 (2011).

[72] T. Mikami, T. Okamoto, K. Kato, "Sellmeier and thermo-optic dispersion formulas for $\mathrm{RbTiOPO}_{4}$," Opt. Mat. 31, 1628-1630 (2009).

[73] W. Tropf, M. E. Thomas, "Infrared refractive index and thermo-optic coefficient measurement at APL," Johns Hopkins APL Tecn. Dig. 19, 293-298 (1998).

[74] OS Heavens : Measurement of optical constants of thin films, - Physics of Thin Films, (Academic Press New York, 1964)

[75] M. Cathelinaud : Les méthodes spectrophotométriques pour la détermination d'indice de couches minces, CNRS/MRCT/ROP Workshop on metrology of refractive indices, Paris, November 2008 and 2009 http://www.rop.cnrs.fr/spip.php?article349

[76] P. Bousquet, F. Flory, and P. Roche : "Scattering from multilayer thin films: theory and experiment" Journal of the Optical Society of America Vol. 71, Issue 9, pp. 1115-1123 (1981)

[77] A. Piegari, F. Flory: Optical thin films and coatings: From materials to applications (Woodhead Publishing, Oxford, Cambridge, 2013)

[78] C. Amra: "Light scattering from multilayer optics. I. Tools of investigation" - JOSA A 11, pp. 197$210(1994)$

[79] S. Adachi: "Model dielectric constants of GaP, GaAs, Gasb, InP, InAs, and Insb", Physical Review B Vol 35, n¹4, 7454-7463, 1987

[80] V. Kumar, S.K. Sharma,T.P Sharma, V. Singh: "Band gap determination in thick films from reflectance measurements" Optical Material 12,115-119, (1999)

[81] P.S. Hauge: "Polycrystalline silicon film thickness measurement from analysis of visible reflectance spectra JOSA, 69(8), 1143-1152 (1979)

[82] J C. Manifacier, J. Gassiot, J P. Fillard:" A simple method for the determination of the optical constants, $n, k$ and the thickness of a weakly absorbing thin film", J. Phys. E Sci. Instrum 9, 10021004, (1976)

[83] I. Ohlidal, K. Navrfitil, and E. Schmidt: "Simple Method for the Complete Optical Analysis of Very Thick and Weakly Absorbing Films", Appl. Phys. A 29, 157-162 (1982)

[84] R. Jacobsson : "Inhomogeneous and coevaporated homogeneous films for optical applications", Physics of Thin Films, (Academic Press, New York,1975) 
[85] J. P. Borgogno, B. Lazarides, and E. Pelletier : "Automatic determination of the optical constants of inhomogeneous thin films" Applied Optics Vol. 21,pp. 4020-4029 (1982)

[86] J.A Dobrowolski, F.C Ho, A. Waldorf :" Determination of optical constants of thin film coating materials based on inverse synthesis", - Applied optics, Vol. 22, Issue 20, pp. 3191-3200 (1983)

[87] F. Abeles: Methods for determining optical parameters of thin films - - in Progress in optics (E. Wolf Ed) vol2, (Elsevier, Amsterdam, 1963)

[88] SG Tomlin:" Optical reflection and transmission formulae for thin film", J. Phys A Appl. Phys 1667-1671, (1968)

[89] H. Wolter: "Zur Optik dünner Metallfilme", Zeitschrift für Physik, Volume 105, Issue 5, pp 269308, May 1937

[90] R. E. Denton, R. D. Campbell and S G Tomlin: "The determination of the optical constants of thin films from measurements of reflectance and transmittance at normal incidence" Journal of Physics D: Applied Physics, Volume 5, Number 4, 852-863, 1972

[91] M. Cathelinaud, F. Lemarquis, C. Amra : "Index determination of opaque and semitransparent metallic films: application to light absorbers", - Applied optics, 2002

[92] M. Cathelinaud , F. Lemarquis, J. Loesel, B. Cousin : "Metal-dielectric light absorbers manufactured by ion plating" Proc. SPIE 5250, Advances in Optical Thin Films, 511 February 25, 2004

[93] B. Badoil, M. Cathelinaud, F. Lemarchand, F. Lemarquis, M. Lequime,"Development of a RealTime Reflectance and Transmittance Monitoring System for the Manufacturing of Metal-Dielectric Light Absorber", Sixth International Conference on Space Optics, Proceedings of ESA/CNES ICSO 2006

[94] G. Hass, L. Hadley : Optical Constants of metals in American Institute of Physics Handbook Gray D E Ed (MCGraw, New York /Londen/ Hill 1972)

[95] Press, W. H., Teukolsky, S. A., Vetterling, W. T., \& Flannery, B. P., Numerical Recipes in FORTRAN: The Art of Scientific Computing (Cambridge Univ. Press, Cambridge) 1992

[96] D. P. Arndt, R. M. A. Azzam, J. M. Bennett, J. P. Borgogno, C. K. Carniglia, W. E. Case, J. A. Dobrowolski, U. J. Gibson, T. Tuttle Hart, F. C. Ho, V. A. Hodgkin, W. P. Klapp, H. A. Macleod, E. Pelletier, M. K. Purvis, D. M. Quinn, D. H. Strome, R. Swenson, P. A. Temple, and T. F. Thonn : "Multiple determination of the optical constants of thin-film coating materials" Applied Optics vol 23 n²0 pp. 3571-3596 (1984)

[97] A Duparré and D. Ristau: “Optical interference coatings" 2007 Measurement problem Applied Optics Vol 47 n¹3 PP C179-C184

[98] A Duparré and D. Ristau: "Optical interference coatings measurement problem" 2013 Applied Optics Vol 53 n4 PP A281-A286 (2014)

[99] F. Lemarchand, C. Deumié, M. Zerrad, L. Abel-Tiberini, B. Bertussi, G. Georges, B. Lazaridès, M. Cathelinaud, M. Lequime, and C. Amra: "Optical characterization of an unknown single layer: Institut 


\section{Biography of Authors}

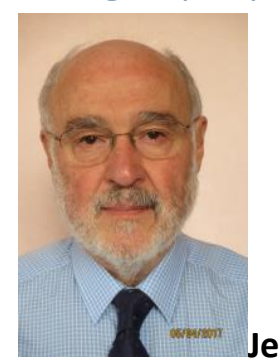

Jean-Louis Meyzonnette was born in 1945. He got his Master's and PhD degrees respectively in 1968 from the Institut d'Optique (Orsay, France) and in 1975 from the Institute of Optics (University of Rochester, USA). From 1975 to 1990, he worked as a research scientist in the Optronics department of then Thomson-CSF (now Thales) Avionics Division, where he was a team leader in the design and test of infra-red, night vision and laser devices for defense applications, a large part of his research being dedicated to the design of coherent laser radars and active imaging sensors. In 1990, sponsored by an association set up by the major French optical companies in order to tighten the ties between the optics/photonics industry and the Institut d'Optique, he joined that academic Institution as a professor in instrumental optics. Since that date, he has been teaching numerous master students and engineers or technicians from industry with courses on optical radiometry, detection, and electrooptical system design. He has also been participating in diverse infrared metrological projects, and advising a dozen PhD candidates in optical instrumentation. He retired from Institut d'Optique in 2012 and acts now as an independent consultant.

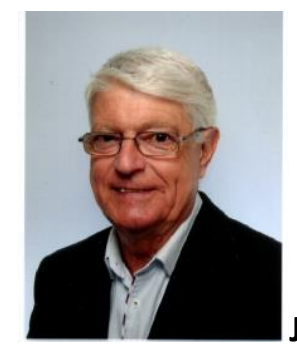

Jacques MANGIN received Ph. D. and Doctorate of Sciences degrees at the University of Nancy, France, in 1975 and 1981 respectively, and where he joined the Far-Infrared Laboratory as Researcher at the National Center of Scientific Research (CNRS). He held a post doctoral position in Lawrence Berkeley National Laboratory, University of California Berkeley in 1984-1985, working in very-low temperature detectors (bolometers) and optical properties of materials at cryogenic temperatures for space applications. In 1996 he joined as research manager the department of Optical Materials at LICB, Burgundy University at Dijon, where he was in charge of the growth of optical crystals, solid-state physics, metrology of thermo- and electro-optical properties of materials and nonlinear optics. He acts currently as scientific expert in several civil and/or defense projects for proper 
implementation of crucial optical components. He is also co-founder of Cristal Laser Company (1990), leader in the development of nonlinear optical crystals like LBO and crystals of the $\mathrm{KTiOPO}_{4}$ family.

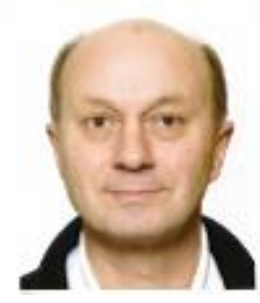

IMichel Cathelinaud CNRS Research Engineer, Ph. D at the University of AixMarseille, France in 2000, has over 20-year experiences in the design, manufacture and characterization of optical coatings for terrestrial and space applications (Fresnel Institute Marseille 1991-2007). From 2005 to 2007, he was responsible of technologic network in optic and photonic of the CNRS. He joined the ISCR in October 2013 to work on chalcogenide thin films after 6-year service as Deputy Head of Mission for Resources and Skills in Technology (MRCT Paris) of CNRS (DGDS / MI) from 2008 to 2013. 